\title{
Regulation of Cell Polarity in the Budding Yeast
}

Saccharomyces cerevisiae

\author{
Dissertation \\ Zur Erlangung des Doktorgrades \\ der Mathematisch-Naturwissenschaftlichen Fakultäten \\ der Georg-August-Universität zu Göttingen
}

\section{Vorgelegt von \\ Naimeh Taheri Talesh}

aus Talesh

Göttingen 2002 
Die vorliegende Arbeit wurde in der Arbeitsgruppe von PD Dr. Hans-Ulrich Mösch in der Abteilung Molekulare Mikrobiologie und Genetik von Prof. Dr. Gerhard H. Braus am Institut für Mikrobiologie und Genetik der Georg-August-Universität Göttingen angefertigt.

Teile dieser Arbeit wurden veröffentlicht in:

Taheri, N., Köhler, T.; Braus, G.H. and Mösch, H.-U. (2000) Asymmetrically localized Bud8p and Bud9p proteins control yeast cell polarity and development. ЕМBO J., 19: 6686-6696.

Taheri, N., Obermayer, A., Braus, G. H. and Mösch, H.-U. (2002) The spatial landmark protein Bud8p links yeast cell polarity to translation. Submitted for publication.

D7

Referent: PD Dr. H.-U. Mösch

Korreferent: Prof. Dr. G. H. Braus

Tag der mündlichen Prüfung: 31.10.2002 
Anahid und Mostafa gewidmet 


\section{Acknowledgements}

I would like to thank PD Dr. Hans-Ulrich Mösch for his excellent supervision and kind assistance of this dissertation as well as for his continuing interest.

Thanks are also due to Prof. Gerhard Braus for his helpful advice and support on many aspects of my research and for agreeing to be co-examiner.

I would like to thank especially Maria Mayer for her excellent technical assistance during the last phase of this work and a very pleasant working atmosphere. Many thanks are also due to Tim Köhler and Olav Grundmann for their kind supports, for the discussions and ideas.

I appreciate the help of Anne Obermayer during her diploma work and for assistance of Kohelia Choudhury and Jens Gerdes during practical work.

Many thanks are due to Melanie Bolte, Tim Köhler, Ralf Pries and Oliver Valerius for critical reading of this thesis, discussions and ideas.

I appreciate all present members of the Department of Molecular Microbiology and Genetics, Katja Anttonen, Ingrid Bahr, Katrin Bömeke, Stefan Brückner, Silke Busch, Patrick Dieckhoff, Oliver Draht, Verena Große, Frau Günzel, Gabriele Heinrich, Kerstin Helmstaedt, Stefan Irniger, Malte Kleinschmidt, Virginia Korte, Sven Krappmann, Heidi Northemann, Andrea Pfeil, Verena Pretz, Nicole Scheiter, Elke Schwier, Axel Strittmatter and Claudia Wagner for their supports and a very agreeable working atmosphere.

Additionally, I would like to thank former colleagues, Meike Andermann, Matthias Bäumer, Katrin Düvel, Sabine Eckert, Markus Hartmann, Bernd Hoffmann, Friedericke Hüttenrauch, Frau Sternbach and Stefanie Wesche for the pleasant working atmosphere.

Finally, I would like to thank my family, especially my daughter Anahid and Mostafa for their patience and moral support. Many thanks are also due to all my friends specially Bärbel and Hossein Mohseninia, and Karen Plain-Switzer who have supported me during my work. 
Table of Contents

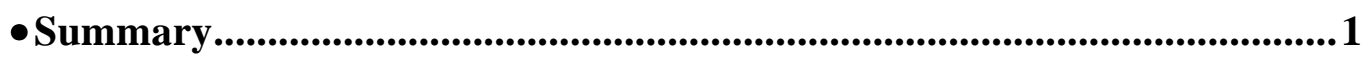

•Zusammenfassung ..............................................................................................2

- Chapter 1:

Introduction . .3

1. Definition and importance of cell polarity .............................................. 3

2. Polarization of cell growth in the budding yeast $S$. cerevisiae ................... 4

2.1. Establishment of polarity states during cell division................................. 4

2.2. The role of the actin cytoskeleton for cell polarization ............................ 5

3. Genetic control of bud site selection in $S$. cerevisiae .............................. 6

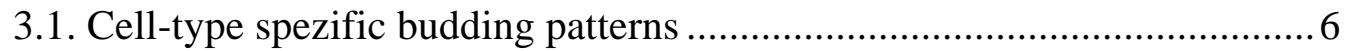

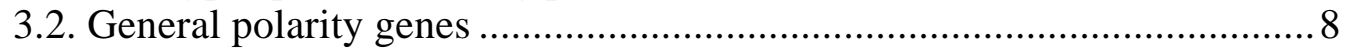

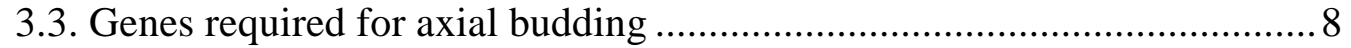

3.4. Genes required for bipolar budding .................................................. 9

3.5. Genes required for unipolar distal budding......................................... 11

4. Molecular machinery for directed cell division in budding yeast ............. 12

4.1. Basic steps of polarity establishment................................................. 12

4.2. Haploid-specific landmark proteins..................................................... 14

4.3. Diploid-specific landmark proteins Bud8p and Bud9p .......................... 16

4.4. Polarity establishment proteins Rsr1p/Bud1p and Cdc42p...................... 17

4.5. Polarization of the actin cytoskeleton by formins and PAKs .................. 18

4.6. Polarized secretion along actin cables ............................................... 18

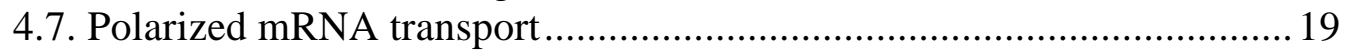

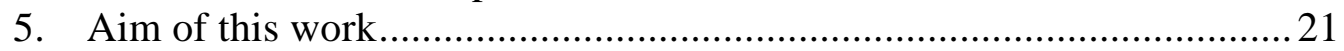

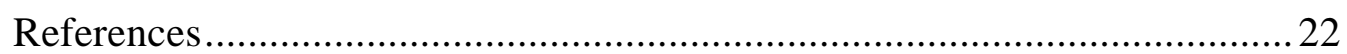

- Chapter 2:

Asymmetrically localized Bud8p and Bud9p proteins control yeast cell

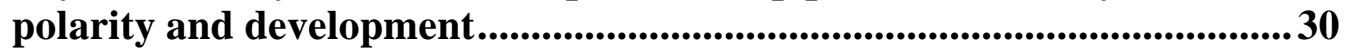

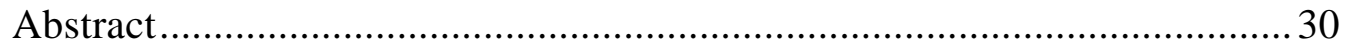

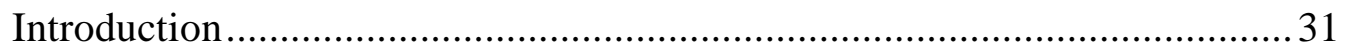

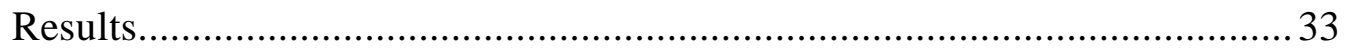

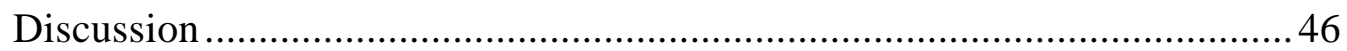

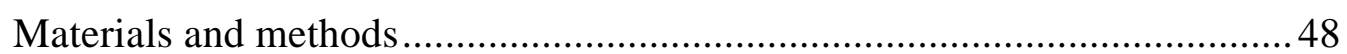

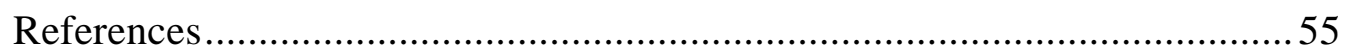

- Chapter 3:

Type I myosin is required for selection of cell division sites in budding yeast and physically associates with the landmark protein Bud8p............58

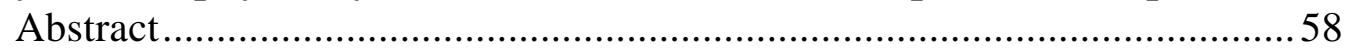

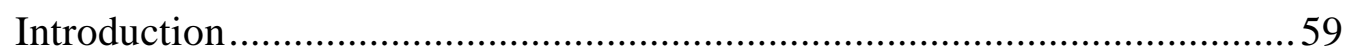

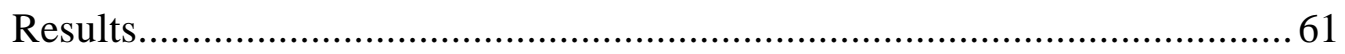

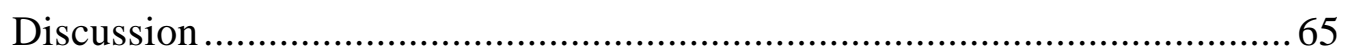

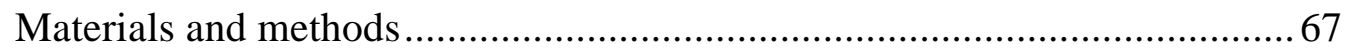

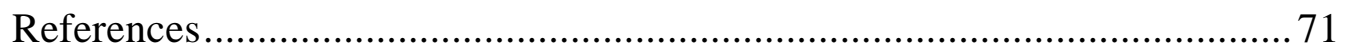


- Chapter 4:

The spatial landmark protein Bud8p links yeast cell polarity to factors involved in cellular transport and translation .....................................................74

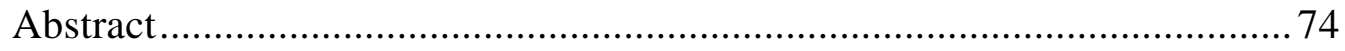

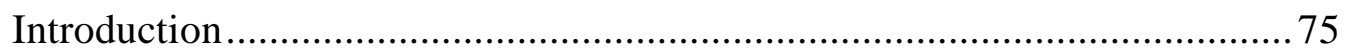

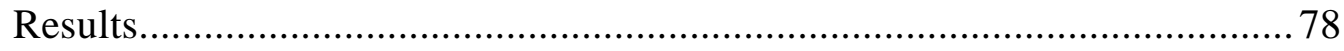

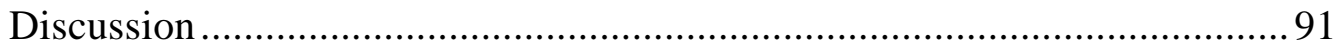

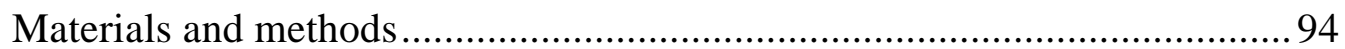

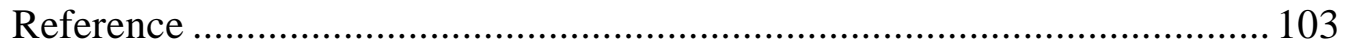

• Curriculum Vitae .........................................................................................107 


\section{Summary}

The budding yeast Saccharomyces cerevisiae establishes cell polarity at several stages during cell division. At the beginning of the division cycle, cells of $S$. cerevisiae choose sites for cell division in specific spatial patterns that are under genetic control of the cell-type and of external conditions. Diploid strains bud in a bipolar pattern, where cells form buds either near their birth site, called the proximal pole, or at the pole opposite to the birth site, called the distal pole. In response to nitrogen starvation, diploid strains switch to a unipolar bud site selection pattern, where buds form predominantly at the distal pole. Selection of division sites is a three-step process that includes (i) marking the division site by spatial landmark proteins, (ii) recognition of the landmark by polarity establishment and actinorganizing proteins, and (iii) polarization of the actin cytoskeleton and direction of secretion towards the site of the landmark to initiate cell division.

In this work, the molecular functions of two spatial landmark proteins, Bud8p and Bud9p, were analyzed in molecular detail. Genetic and cell biological analysis revealed that Bud8p is localized at the distal cell pole and is required for selection of the distal cell pole as division site under all conditions. Bud9p is localized at both poles, but is required for selection of the proximal pole only. In contrast to Bud8p, Bud9p is prevented from being delivered to the distal pole by nitrogen starvation. Biochemical analysis showed that Bud8p and Bud9p associate in vivo. Thus, Bud8p is likely to be a landmark protein for bud initiation at the distal pole, whereas Bud9p inhibits cell division at the distal pole, but serves as a landmark protein at the proximal pole.

In order to test the role of actin-organizing proteins in the process of bud site selection, genetic and physical interactions between the landmark protein Bud8p and Bud9p and the yeast type I myosins Myo3p and Myo5p were analyzed. Both, Myo3p and Myo5p are required for bud site selection that is directed by Bud8p and Bud9p. Biochemical analysis revealed that Bud8p associates with Myo3p in vivo. This suggests, that landmark proteins interact with type I myosins, in order to induce sitespecific actin assembly and cell growth.

Novel interaction partners for Bud8p were isolated by the yeast two-hybrid system, and physical interactions were verified by biochemical co-purification experiments. Surprisingly, proteins involved in cellular transport and translation were identified to interact with Bud8p and to control bud site selection including the ribosomal protein Rpl12Ap, the RNA-binding and polysome-associated vigilin protein Scp160p and Trs120p, a factor involved in directed vesicular transport. Thus, the landmark protein Bud8p might control directed cell division by interaction with components that regulate cellular transport and site-specific translation. 


\section{Zusammenfassung}

Zellen der Bäckerhefe Saccharomyces cerevisiae werden $\mathrm{zu}$ verschiedenen Zeitpunkten während der Zellteilung polarisiert. Zu Beginn der Teilung werden spezifische Orte für die Sprossung ausgewählt. Die Wahl der Sprossungsorte folgt definierten räumlichen Mustern und unterliegt der Kontrolle von Zelltyp und Nährstoffbedingungen. Diploide Hefezellen folgen bei der Sprosswahl einem bipolaren Muster, bei dem die Zellteilung mit gleicher Wahrscheinlichkeit entweder am Geburtsende, dem proximalen Zellpol, oder am direkt gegenüber liegenden Ort, dem distalen Zellpol beginnt. Bei Stickstoffmangel wechseln diploide Zellen zu einem unipolar-distalen Sprossungsmuster. Die Wahl des Sprossungsortes erfolgt in drei Schritten. (i) Der Sprossungsort wird markiert durch spezifische Zellpolproteine, die (ii) von weiteren Polaritätsproteinen erkannt werden, die ihrerseits (iii) das Aktinzytoskelett und die intrazelluläre Sekretion auf die Polproteine ausrichten.

In dieser Arbeit wurden die Funktionen von zwei Zellpolproteinen, Bud8p und Bud9p, im Detail analysiert. Genetische und zellbiologische Analysen zeigten, dass Bud8p am distalen Zellpol lokalisiert ist und für die Wahl des distalen Zellpols benötigt wird. Bud9p ist an beiden Zellpolen zu finden, wird aber nur für die proximale Sprossung benötigt. Biochemische Untersuchung zeigten zudem, dass Bud8p und Bud9p (vermutlich am distalen Pol) im Komplex vorliegen. Bud8p fungiert deshalb vermutlich als Markerprotein für die distale Sprosswahl und wird dabei durch die Anwesenheit von Bud9p negativ beeinflusst. Bud9p hat zusätzlich eine positive Funktion als Markerprotein für die Wahl des proximalen Zellpols.

Um die Funktion von Regulatoren des Aktinzytoskeletts bei der Sprosswahl zu untersuchen, wurden genetische und biochemische Interaktionen zwischen den Polproteinen Bud8p und Bud9p und den Typ I Myosinen Myo3p und Myo5p analysiert. Sowohl Myo3p als auch Myo5p werden für die Erkennung der Zellpole benötigt. Zusätzlich bindet Bud8p an Myo3p, was darauf hindeutet, dass Typ I Myosine durch Interaktion mit Polproteinen die Sprosswahl durch ortspezifische Aktinpolymerisierung steuern könnten.

Neue Interaktionspartner von Bud8p wurden mit dem Hefe-Two-Hybrid-System isoliert und über biochemische Co-Reinigungsexperimente weiter charakterisiert. Dabei wurden einige Proteine als Bud8p-Bindepartner identifiziert, die an der Translation oder am zellulären Transport beteiligt sind, z.B. das ribosomale Protein Rpl12Ap, das Polysomen-assozierte und mRNA-bindende Protein Scp160p, sowie das am Vesikeltransport beteiligte Protein Trs120p. Diese Befunde lassen vermuten, dass Bud8p die Sprosswahl durch Beeinflussung des zellulären Transports und der Translation regulieren könnte. 


\section{Chapter 1}

\section{Introduction}

\section{Definition and importance of cell polarity}

Eukaryotic cells respond to intracellular and extracellular cues to direct cell growth and division. Cell polarity is most simply defined as an asymmetric distribution of specific proteins, nucleic acids, macromolecular assemblies and organelles near a defined spatial site, thereby allowing the cell to increase its surface in an asymmetrical or polar fashion. Cell polarity is fundamentally important for differentiation, proliferation, morphogenesis, and function of unicellular and multicellular organisms. Directional cell divisions occur during the life cycle of many distinct organisms. In the bacteria Caulobacter crescentus and Bacillus subtilis, differentiation is a result of asymmetric cell division (Hofmeister and Brun, 2000). In the yeasts Saccharomyces cerevisiae and Schizosaccharomyces pombe, cell polarization is essential to division and mating (Bähler and Peter, 2000). In the filamentous fungi Neurospora crassa and Aspergillus nidulans, polarized growth is a regulated cellular expansion that underlies the hyphae formation process. (Gow, 1996). In insects, e. g. Drosophila melanogaster, and in mammals cell polarity plays an important role in oogenesis, embryogenesis, neurogenesis and often involves asymmetric messenger RNA (mRNA) transport (Jansen, 1999 ; Jansen, 2001).

Study of model organisms is a promising avenue to uncover general molecular mechanisms that underlie the establishment and maintenance of a polarized cell. The budding yeast $S$. cerevisiae is an excellent model system for the study of polarized cell growth and establishment of cell polarity. Molecular genetics and biochemical studies in this organism have contributed to getting a first insight into the framework of genes and gene products that control cell polarity in eukaryotes. In recent years, a large number of proteins have been identified to be involved in the establishment and maintenance of cell polarity, among them many regulatory and cytoskeletal components. In addition, a number of proteins have been identified, whose mechanism of action is not well understood.

This introductory chapter summarizes recent advances on the molecular machinery that controls establishment and maintenance of cell polarity in S. cerevisiae. 


\section{Polarization of cell growth in the budding yeast $S$. cerevisiae}

\subsection{Establishment of polarity states during cell division}

Yeast cells divide by budding that confers a unique relationship between cell growth and the sequence of events that constitute the cell division cycle. Progression through the cell division cycle is divided into several phases, and yeast cells establish polarity at several stages during cell division (Figure 1).

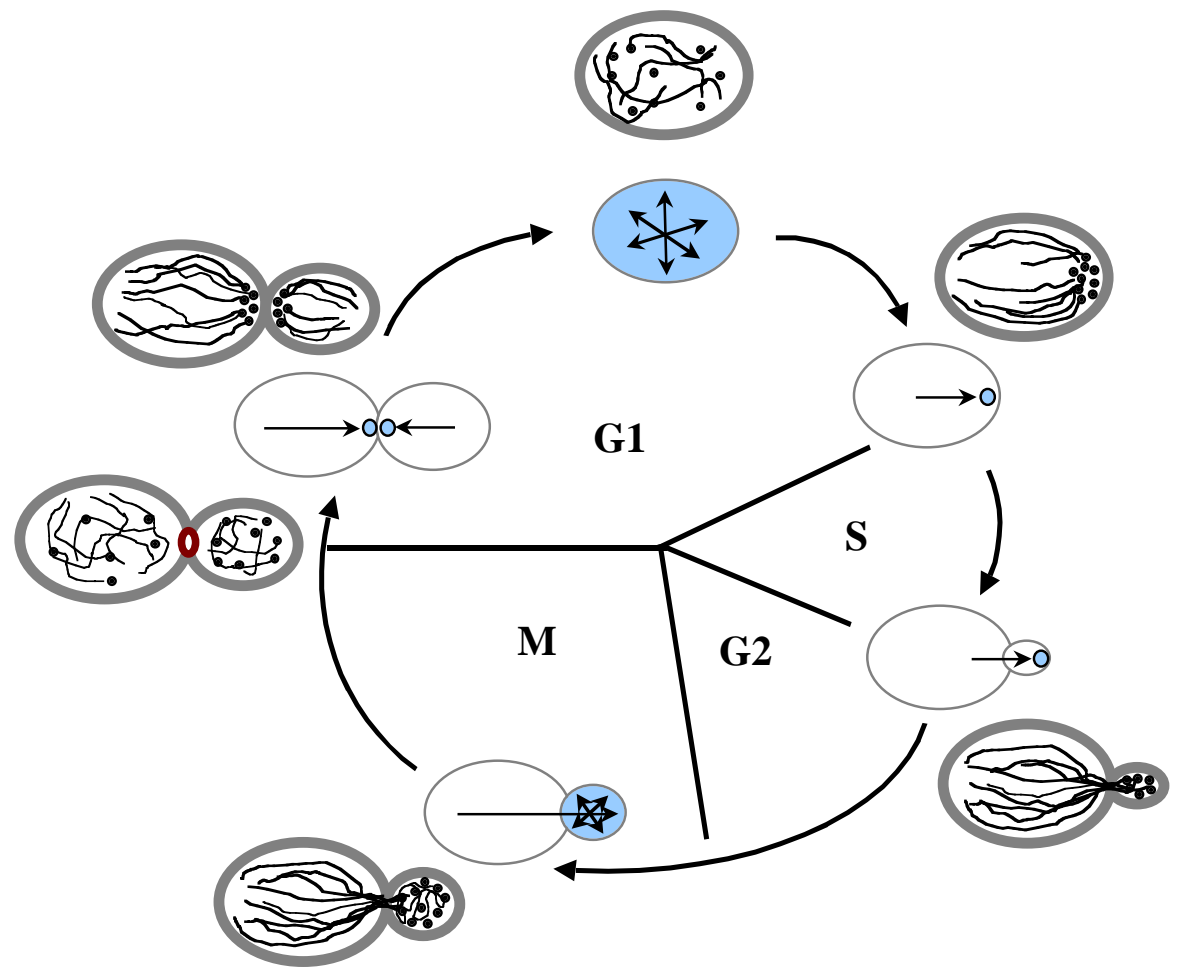

Figure 1. Cell divisions and establishment of cell polarity in budding yeast by a polarized actin cytoskeleton throughout the cell cycle. The cell cycle begins in G1 (START) to select a bud site. In this phase, a cap (blue dots) of regulatory and cytoskeletal proteins establishes the polarity of actin cables (black filaments) and cortical patches (black dots). During $\mathrm{S}$ phase, where the cell grows apically, actin cables extend from the mother cell into the bud. Tight localization of the cap orients actin cables. Actin cables direct secretory vesicles to the cap, where they accumulate (blue) and fuse, thus polarizing growth (arrows). During isotropic growth (G2-M), the proteins of the cap are more diffusely distributed, cortical patches are isotropically distributed, and actin cables form a meshwork. In $\mathrm{M}$ phase, the bud separates from mother cell, where an additional cytoskeletal structure, a cytokinetic ring, mediates cell division (red).

In the G1 phase, the cell remains unbudded until there are sufficient nutrients for cell division to begin, at "START". Polarization begins towards the site for cell division and involves non-essential and essential steps. In a first non-essential step, yeast cells select a bud site by spatial control. In a second and essential step, the cell initiates division and starts to emerge a bud. 
During S phase, the cell shows apical growth that means polarized growth towards the apex of the bud. In G2 phase, cell growth switches to isotropic growth. Timing of the switch from apical to isotropic growth controls cell morphology. A prolonged polarized phase leads to a long cell morphology. Short or too short apical growth phases lead to round cells. The time period of polarized cell growth also affects bud site selection, because it influences the deposition of (non-essential) spatial cues that regulate bud site selection (discussed below in sections 3.1 - 3.3). In $\mathrm{M}$ phase, the cytoplasm of the bud separates from that of the mother cell (cytokinesis) and the bud itself separates from the mother cell.

\subsection{The role of the actin cytoskeleton for cell polarization}

Virtually all aspects of cell polarization derive from polarization of the actin cytoskeleton. Therefore, regulation of cell polarization involves a careful and elaborate control of the actin cytoskeleton during all phases of the yeast division cycle. The yeast actin cytoskeleton is organized into four biochemical and morphological structures: the cap, actin cables, cortical patches, and a cytokinetic ring (Adams and Pringle, 1984; Chant and Pringle, 1995; Lew and Reed, 1995). The cap consists of a polarized accumulation of cytoskeletal proteins and regulatory proteins, actin cables are long bundles of actin filaments, cortical patches are discrete cytoskeletal bodies, and the cytokinetic ring is a set of proteins which affect cell division or separation. The cap, actin cables and cortical patches, all reside at the cell cortex in a polarized distribution that correlates with growth (Adams and Pringle, 1984; Lew and Reed, 1995; Amberg, 1998) (Figure 1). At the beginning of the cell cycle, the cap is localized at the growing tip of the cell and overlaps with a cluster of cortical patches. In addition, actin cables extend from the mother cell into the bud. As a result, the bud grows apically. During the apical isotropic switch, cap components and patches redistribute over the bud surface. During isotropic growth, cables extend from the mother cell into a network in the bud. Finally, after cytokinesis, all three actin components reorient to the mother-bud junction (Pruyne and Bretscher, 2000a; Pruyne and Bretscher, 2000b).

Molecular analysis has identified many components of the cap, the actin cables and the cortical patches. The cap includes proteins involved in the Rho-GTPase- and CDK (cyclin-dependent protein kinase)-signaling pathways, insist on Cdc42p, Cdc24p, Bem1p, Ste20p, Cla4p, Cdc28p, and polarisome proteins Bni1p, Spa2p and Bud6p. These cap proteins are thought to function as nucleation and anchor sites for actin cables, because cables orient towards the cap proteins during all phases of 
growth (Figure 1). Actin cables are necessary for several events important for cell polarization, including Myo2p (a type $\mathrm{V}$ myosin)-dependent polarized delivery of membrane vesicles that contain polarity proteins such as Bud6p or Bnilp (Jin and Amberg, 2000; Ozaki-Kuroda et al., 2001), transport of late Golgi elements into the bud (Rossanese et al., 2001), and asymmetric transport of mRNA for the cell fate determinant ASH1 by the type V myosin, Myo4p (Takizawa et al., 1997). Actin patches are motile structures associated with plasma membrane invaginations that concentrate at sites of active growth and cell wall deposition. Polarized actin patches may also be involved in endocytosis. A lot of mutants defective in endocytosis were also found to have defects in actin cytoskeleton organization (Goode and Rodal, 2001; Goode et al., 2001; Jeng and Welch, 2001; Munn, 2001). Many actin-binding proteins interact with endocytic components (Goode and Rodal, 2001; Goode et al., 2001). A key regulator of actin patch assembly is the Arp $2 / 3$ complex, which is regulated by different mechanisms involving different proteins (Goode et al., 2001). These proteins include Las17p/Bee1p functioning with the type I myosins Myo3p and Myo5p (Evangelista et al., 2000; Lechler et al., 2001; Lechler et al., 2000; Winter et al., 1999), and the two actin-binding proteins Pan1p and Abp1p (Duncan et al., 2001; Goode et al., 2001). Type I myosins perform further essential functions in endocytosis and actin organization and are required for actin assembly in the permeabilized cell assay (Lechler et al., 2000). The cytokinetic ring includes the septins Cdc3p, Cdc10p, Cdc11p and Cdc12p that are involved in cytokinesis and bud site selection. The function of septin structure is important for maintenance of cell polarity by formation of a cortical boundary between the bud and mother cell during isotropic bud growth. Septins are further required for reorientation of cortical patches and actin cables to the mother-bud neck after bud growth in order to complete cell separation (Casamayor and Snyder, 2002; Pruyne and Bretscher, 2000a; Pruyne and Bretscher, 2000b).

\section{Genetic control of bud site selection in $S$. cerevisiae}

\subsection{Cell-type specific budding patterns}

During its life cycle, $S$. cerevisiae can adapt distinct cell-types that are represented by the haploid and the diploid yeast forms, in which the organisms lives as individual single cells. Diploid strains are also able to switch to a multicellular growth form, where $S$. cerevisiae grows as linear filaments of pseudohyphal cells (Kron et al., 1994). 
Although selection of budding sites is non-essential for cell division, it appears to be of great importance for free-living yeasts, because yeast cells have maintained highly regulated and sophisticated control mechanisms of budding patterns that depend on the cell-type and the environmental conditions (Figure 2).

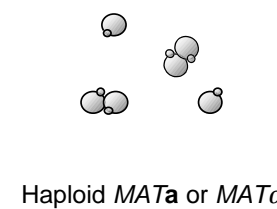

Haploid MATa or MAT $\alpha$

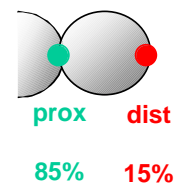

axial

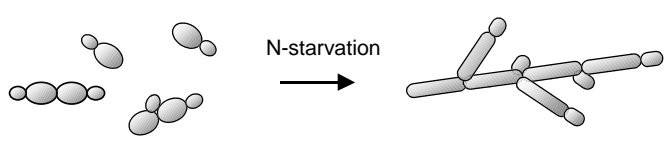

Diploid MATa/MATo

YF

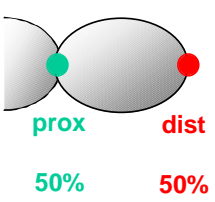

bipolar
Diploid MATa/MAT $\alpha$

PH

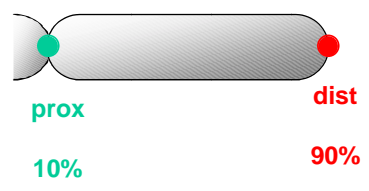

unipolar distal

Figure 2. Bud site selection patterns of different cell types. Haploid cells preferentially form buds in an axial pattern at the proximal pole (green circle), the site of their birth. Diploid yeast form cells (YF) bud in a bipolar pattern, where buds form at both poles, either at proximal or at distal pole (opposite to birth pole of the mother cell) (red circle). Diploid pseudohyphal cells (PH) bud in a unipolar distal budding pattern, where most buds emerge at the distal pole.

Haploid $\mathbf{a}$ and $\alpha$ cells divide in an axial pattern, in which the mother and daughter cells are constrained to form their buds immediately adjacent to the previous site of cell separation. Diploid yeast form cells divide in a bipolar pattern, in which mother and daughter cell bud either adjacent to the birth site (proximal pole) or opposite the birth site (distal pole). New mother cells usually bud at proximal sites, whereas cells that have undergone one or more divisions exhibit an increased preference for distal sites. Diploid pseudohyphal cells preferentially bud in a unipolar distal pattern, where most of the cells bud at the distal pole (Gimeno et al., 1992; Kron et al., 1994). The unipolar distal budding process is a prerequisite for the establishment of filamentous structures, which is regulated by nutritional signals and guides the direction of the growing $\mathrm{PH}$ filaments. 


\subsection{General polarity genes}

Genetic analysis has identified three distinct classes of genes that are required for bud site selection of the distinct cell-types. A first class of genes is required for all three axial, bipolar and unipolar distal budding patterns. A second class includes genes specifically required for the axial pattern of haploid, and a third class is required for the bipolar and unipolar distal pattern of diploids.

The first class of genes includes BUD1/RSR1, BUD2 and BUD5 (Chant, 1999). Mutations in these genes display a random budding pattern in all cell-types. These genes code for the Bud1p/Rsr1p, Bud2p and Bud5p proteins, which constitute a GTPase (Ras-related GTPase) signaling module that is thought to recruit the components of cell polarity establishment machinery and the actin cytoskeleton. The Bud1p/Rsr1p signaling module may direct the bud formation components to cortical tags at future bud sites in both haploid and diploid cells (Chant, 1999) (discussed in sections 4.2, 4.3 and 4.4).

\subsection{Genes required for axial budding}

AXL1, BUD10/AXL2, BUD3 and BUD4 (Chant and Herskowitz, 1991; Fujita et al., 1994; Halme et al., 1996; Roemer et al., 1996) are genes specifically required for the axial bud site selection pattern in haploid cells. Mutations of these genes result in a bipolar budding pattern. The products of these genes are involved in marking the mother-bud neck during one cell cycle as a site for budding in the next cell cycle. Cells that exhibit axial budding need a cytokinesis tag in which a component at the cytokinesis site persists into the next cell cycle and directs formation of a new bud at an adjacent site (Chant and Herskowitz, 1991; Madden and Snyder, 1998; Snyder et al., 1991). Bud3p (Chant and Herskowitz, 1991; Chant et al., 1995), Bud4p (Chant and Herskowitz, 1991; Sanders and Herskowitz, 1996), and Ax12p/Bud10p (Halme et al., 1996; Roemer et al., 1996) are components of this cortical tag (discussed in section 4.2). $C D C 3, C D C 10, C D C 11$, and $C D C 12$ encode related proteins called septins that localize to the mother-bud neck and are required for cytokinesis and axial budding pattern (Longtine et al., 1996; Roemer et al., 1996). Strains containing temperature-sensitive mutations in septin genes display defects in cytokinesis and axial bud site selection pattern, and instead those cells bud at distal sites (Adams and Pringle, 1984; Chant et al., 1995; Flescher et al., 1993; Madden and Snyder, 1998). 


\subsection{Genes required for bipolar budding}

A large number of genes have been identified that are required for bipolar bud site selection but not for the haploid axial pattern (Costigan and Snyder, 1994; Drubin and Nelson, 1996; Herskowitz, 1995; Pringle et al., 1995). Initial genetic studies have shown that many of these genes encode actin-binding proteins, e.g. the formin protein Bnilp and putative regulators of the actin cytoskeleton, consisting of the proteins Spa2p, Pea2p and Bud6p. Deletion of BNI1, SPA2, PEA2 and BUD6 results in a random budding defect. Bni1p, Spa2p, Pea2p and Bud6p form a complex, the $12 \mathrm{~S}$ polarisome that localizes to sites of polarized growth (Fujiwara et al., 1998; Sheu et al., 1998). These proteins are present as a patch at the incipient bud site, at the tip of the growing bud, and at the mother-bud neck region before cytokinesis (Amberg et al., 1997; Evangelista et al., 1997; Gehrung and Snyder, 1990; Kohno et al., 1996; Snyder, 1989; Snyder et al., 1991; Valtz and Herskowitz, 1996; Zahner et al., 1996). Bni1p, Spa2p, Pea2p and Bud6p are each required for apical growth (Sheu et al., 2000), which is the initial phase of bud growth in which cells grow at the bud tip (Lew and Reed, 1993). It has been suggested that these proteins are required for the polarized deposition of the distal tag during this period (Sheu et al., 2000).

Three genes BUD8, BUD9, and STE20 when mutated, cause diploid cells to form buds at one pole (Sheu et al., 2000; Taheri, et al. 2000; Zahner et al., 1996). Mutants carrying $B U D 8, B U D 9$ or $S T E 20$ deletions display unipolar budding pattern in yeastform. Mutations in the $B U D 8$ gene result in unipolar proximal budding in which mother cell buds preferentially at the proximal pole instead of the distal pole (Mösch and Fink, 1997; Taheri et al., 2000; Zahner et al., 1996). In contrast, bud9/bud9 mutant cells bud at the distal cell pole (unipolar distal). ste20/ste20 cells bud also at the proximal pole of the daughter cell as bud8 diploid mutant strains. Bud8p and Bud9p have been proposed to act as bipolar landmarks or tags that recruit components involved in bud formation (Harkins et al., 2001; Taheri et al., 2000; Zahner et al., 1996). Ste20p is a PAK protein kinase homologue that has been shown to lie in the same genetic pathway as Bud8p (Sheu et al., 2000). In addition, Ste20p and Bud8p have been shown to interact in yeast two-hybrid system (Drees et al., 2001).

A large number of genes were further identified in a systematic genome-wide screen to be involved in bipolar budding in diploid yeast cells (Ni and Snyder, 2001). This screen identified 130 non-essential genes that fell into 11 functional classes including genes involved in actin-cytoskeleton organization, general bud site 
selection, cell polarity, vesicular transport, cell wall synthesis, protein modification, transcription, nuclear function, translation, and other functions (Figure 3).

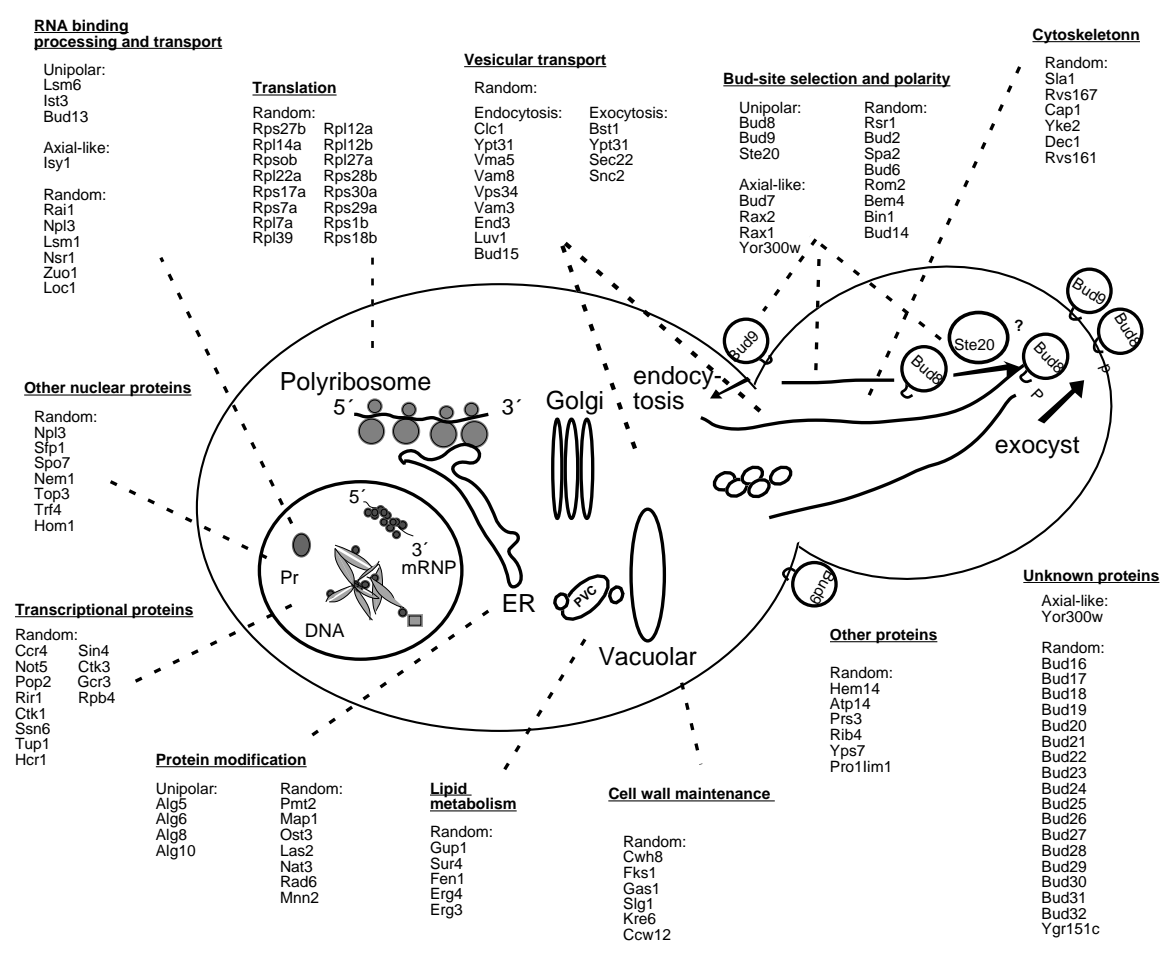

Figure 3. A model for bipolar bud site selection in yeast. The different bud site selection proteins are indicated in the diagram. ER, endoplasmic reticulum; mRNP, mRNA-protein; Pr, proteins; PVC, prevacuolar compartment.

Although this systematic study has identified many novel genes required for bipolar budding, their functions in the process of bud site selection in most cases is not clear. It has been hypothesized that many of these genes might affect expression, modification or transport of spatial cues such as Bud8p and Bud9p (Sheu et al., 2000). Twenty novel BUD genes (BUD13-BUD32) were found that produce either a random budding phenotype upon deletion, or an axial-like budding defect. The localization of the distal tag Bud8p was found to be altered in several of these mutants. The $A L G 5, A L G 6, A L G 8$, and $A L G 10$ genes encode proteins which are involved in yeast dolichol pathway that synthesizes the dolichol-linked oligosaccharide precursor for N-linked proteins glycosylation (Burda and Aebi, 1998; Heesen et al., 1994; Reiss et al., 1996; Stagljar et al., 1994). Mutations in these genes cause a partial unipolar distal budding pattern (Ni and Snyder, 2001). 
Bud8p-GFP shows an increased localization to the mother-bud neck in most of alg mutants compared to wild-type cells. Mutants lacking the LSM6 gene also display a unipolar distal bud site selection pattern. The Lsm6p protein is involved in mRNA splicing (Tharun et al., 2000). The genes including BUD7, RAX2, RAX1, ISY1, and $Y O R 300 w$ are another group of genes that are required for maintenance of the bipolar bud site selection. Mutants lacking these genes display an axial-like or unipolar proximal phenotype. Isylp is a pre-mRNA-splicing factor that is required for splicing in vivo (Dix et al., 1999). This genome-wide screen identified many further genes that upon deletion induce a strong random budding phenotype and encode many proteins that are involved in translation, vesicular transport and regulation of the actin cytoskeleton (Figure 3). Bud8p-GFP localization is abnormal in four vesicular transport mutants including clc1/clc1, ypt31/ypt31, vam3/vam3, and vam8/vam8. Additionally, several mutants with random budding pattern were characterized that include cell wall, lipid metabolism, protein modification, transcription factors and chromosomal proteins, and nuclear proteins. Two cell wall proteins appear to be required for localization of Bud8p, because Bud8p-GFP could not be detected in corresponding $c w h 8 /$ cwh 8 and gasl/gas 1 mutant cells.

\subsection{Genes required for the unipolar distal budding}

In response to nitrogen starvation, diploid yeast cells switch their budding pattern from bipolar to unipolar distal where buds form as pseudohyphae. Therefore, pseudohyphal development is an ideal model to study factors that control oriented cell division in response to external signals. However, the molecular mechanisms that control this change in cell polarity are only little understood, because most studies have addressed the function of genes controlling bud site selection under nutrient-rich conditions, where $S$. cerevisiae will grow and divide as single YF cells. Only a few studies have aimed at identification of genes required for the unipolar distal pattern of the pseudohyphal growth form.

Early studies have shown that Rsr1p/Bud1p is required for pseudohyphal development, because expression of a dominant negative form of $R S R 1 / B U D 1$, $R S R 1^{A s n 16}$, suppresses filament formation in response to nitrogen starvation (Gimeno et al., 1992). Furthermore, full deletion of RSRI/BUDl completely abolished filamentous growth (Taheri et al., 2000). Several bipolar specific bud site selection genes including $B U D 8, B N I 1, P E A 2 / D F G 9$, and $S P A 2$ were identified in a genetic screen to be specifically required for pseudohyphal development (Mösch and Fink, 1997) (Figure 4). This suggests that the pseudohyphal polarity switch might be achieved by alteration of components that control bipolar budding. 
A

YF

Bipolar budding

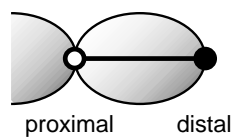

$50 \% \quad 50 \%$

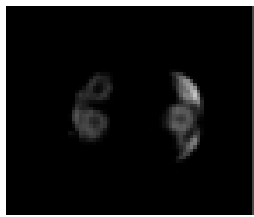

$\mathrm{PH}$

Unipolar distal budding

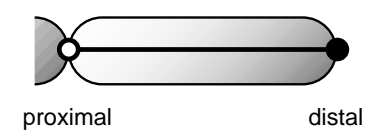

$10 \%$

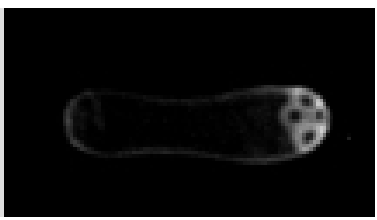

B

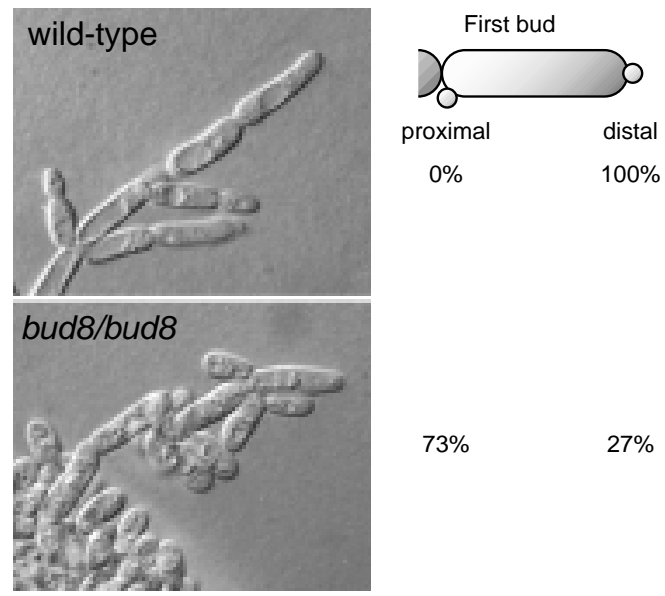

Figure 4. Regulation of pseudohyphal cell polarity in S. cerevisiae. (A) Budding patterns of YF and PH cells. YF cells bud in a bipolar pattern, where buds form with equal (50\%:50\%) probability at either the proximal or the distal cell pole. PH cells prefer the unipolar distal budding pattern, where most buds $(90 \%)$ emerge at the distal pole. Photographs above show distribution of bud scars of YF and $\mathrm{PH}$ cells that were stained with calcofluor and visualized by fluorescence microscopy. (B) Unipolar distal budding of PH requires the BUD 8 gene. Wild-type and bud $8 /$ bud 8 mutant strains were analyzed for selection patterns of first buds of virgin PH cells. Numbers indicate the percentage of virgin PH cells that produced their first bud at the proximal or the distal pole, respectively. After 3 days of growth, PH development of cells at the edges of the colonies was visualized under the microscope using Normaski optics.

\section{Molecular machinery for directed cell division in budding yeast}

\subsection{Basic steps of polarity establishment}

Genetic studies have defined numerous genes that are involved in the distinct budding patterns. However, polarity establishment can be divided into three basic steps independently of the cell type and the budding pattern (Figure 5). First, the site of polarization is marked by landmark proteins. In a second step, the landmark is recognized by a series of further proteins that are collecting called polarity establishment proteins or actin-organizing complex. In a last step, the polarity establishment proteins recruit the machinery required to organize and polymerize the actin cytoskeleton. The polarized cytoskeleton then targets exocytosis or secretion towards the landmark, leading to polarized growth. 
1. Marking the site of polarization

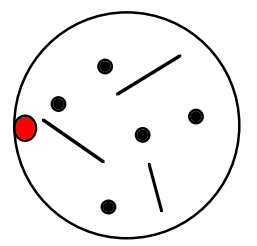

\section{Recognizing the marked site and signalling to the cytoskeleton (polarity establishment)}

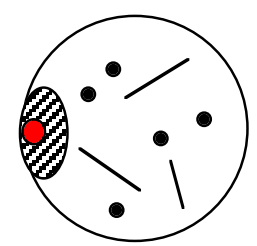

\section{Polarizing the cytoskeleton}

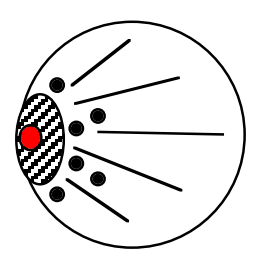

Figure 5. Basic steps of polarity establishment. Establishment of cell polarity requires that cells choose a spatial site (landmark) on their cell cortex (red circle): the position of this site can be defined genetically or in response to internal or external signals. Once a site of polarization has been chosen, this landmark recruits a number of proteins, collectively termed polarity establishment proteins or actin-organizing components (hatched circle). These proteins localize to the landmark and in turn organize the cytoskeleton by polarization of actin patches (black circle) and cables (black filaments).

This simple three-step model might allow to elucidate the molecular functions of the distinct gene products that have been identified by genetic analysis. Several central questions must be answered, before an accurate molecular model for the machinery that directs cell division can be established. First, identity and function of the components for the distinct landmarks must be determined. Here, genes that confer the cell-type specific budding patterns are likely candidates. Secondly, the molecular mechanisms, by which the polarity establishment proteins are recruited to the distinct landmarks and organize the structure of the actin cytoskeleton must be elucidated. Finally, the mechanisms that control asymmetric transport of proteins and RNAs along the polarized actin cytoskeleton to the cell poles must be determined in molecular detail. An interesting question is, whether the components that build the landmarks to initiate site specific cell division might also serve as cortical anchors for the machinery that transports specific proteins and RNAs to the cell poles. 


\subsection{Haploid-specific landmark protein}

Initial genetic analysis identified the septins, BUD3, BUD4, AXL1 and $A X L 2 / B U D 10$ to be specifically required for the axial budding pattern in haploid cells but not for the bipolar pattern of diploid cells (Chant and Herskowitz, 1991; Fujita et al., 1994; Halme et al., 1996; Roemer et al., 1996). Because Bud3p, Bud4p, and Ax12p/Bud10p proteins all localize to the mother-bud neck, they are thought to be part of the axial landmark of haploids (Chant et al., 1995; Halme et al., 1996; Roemer et al., 1996; Sanders and Herskowitz, 1996). Ax12p/Bud10p is a single-pass transmembrane protein with a 500-amino acid extracellular domain and a 300-amino acid intracellular domain (Halme et al., 1996; Roemer et al., 1996). The extracellular domain of Bud10p, which is highly $o$-glycosylated by the mannosyltransferase transferase Pmt4p (Sanders et al., 1999) appears to anchor the protein in the cell wall with Bud3p and Bud4p serving to tightly cluster Bud10p to generate a potent signal (Halme et al., 1996; Roemer et al., 1996). Therefore, Ax12p/Bud10p may act as a polar landmark in axial haploid cells. The mechanism, by which these proteins communicate with downstream machinery to polarize cellular components, is unknown. The most favored view is that the axial spatial cue initiates the local activation of the Bud1p/Rsr1p GTPase, which in turn activates a Rho-like GTPase, Cdc42p (Figure 6). Possibly, the cytoplasmic domain of Ax12p/Bud10p acts to localize one or more Bud1p/Rsr1p regulators, the Bud5p GEF (guanine nucleotide exchange factor) (Chant et al., 1991) or the Bud2p GAP (GTPase-activating protein) (Park et al., 1993). Bud1p/Rsr1p GTPase, which is closely related to the Rasp GTPase subfamily, appears to be uniformly localized to the plasma membrane with little significant soluble pool (Michelitch and Chant, 1996; Park et al., 1997). Bud5p physically interacts with Ax12p/Bud10p (Kang et al., 2001). Targeting of Bud1p/Rsr1p regulators by Ax12p/Bud10p or associated proteins would allow localized Bud1p/Rsr1p activation by GTP binding or GTP/GDP cycling. Activated Bud1p/Rsr1p could then communicate further downstream via actin cytoskeleton polarizing proteins, Cdc24p and Cdc42p. 


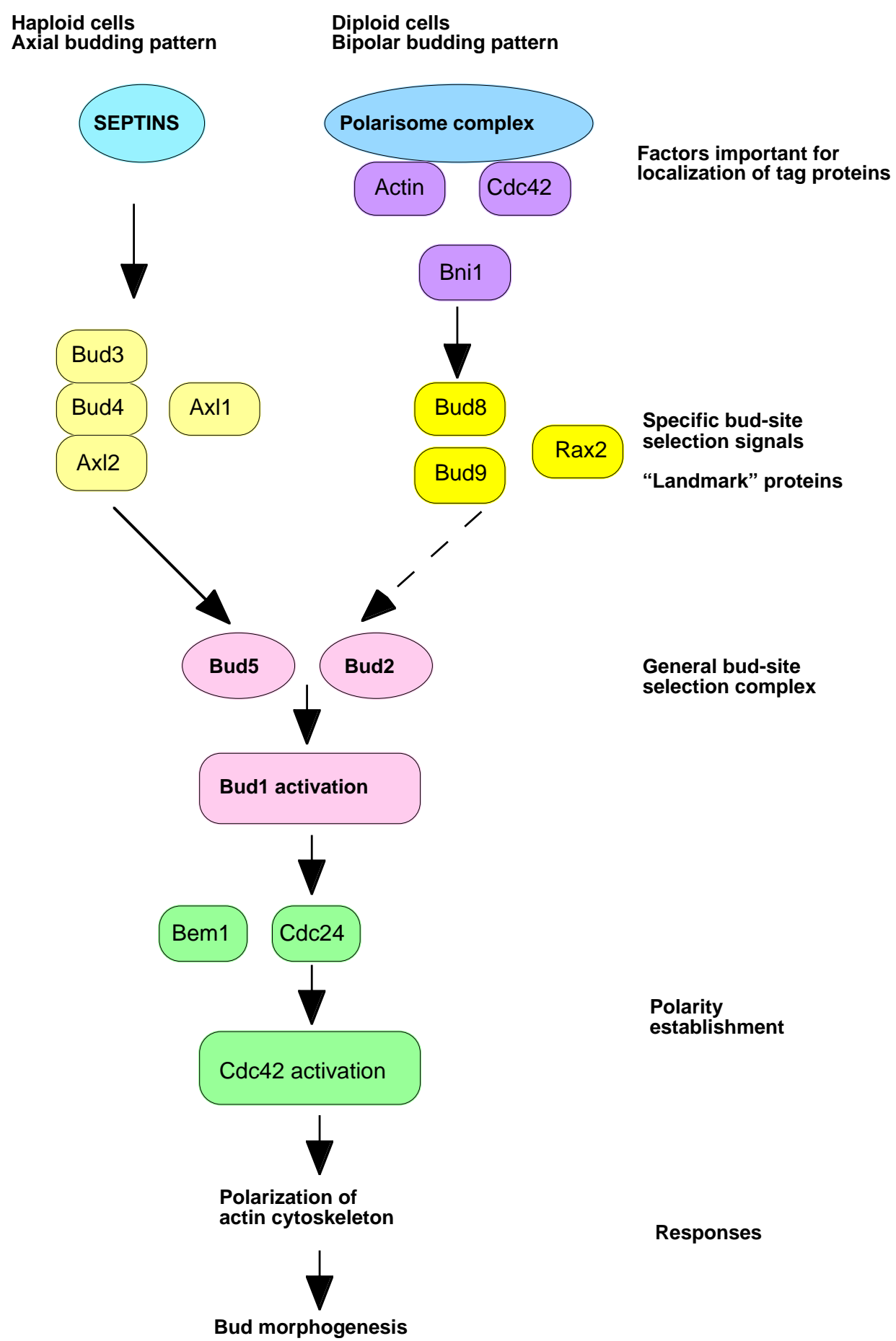

Figure 6. Regulation of actin cytoskeleton polarization and bud growth in yeast cells in response to cortical landmarks. The pathway of actin cytoskeleton polarization and bud emergence by polarity establishing proteins in haploid and diploid cells. The dotted arrow represents hypothetical regulation of proteins by the specific bud-site selection signals in diploid cells. 


\subsection{Diploid-specific landmark proteins Bud8p and Bud9p}

Diploid yeast cells use spatial cues for producing the bipolar budding pattern that are entirely distinct from those used in the axial budding. Previous studies have shown that the bipolar landmarks exist at the poles of diploid cells and that they are persistent for numerous cell cycles (Chant, 1999; Chant and Pringle, 1995). Genetics and cell biological studies have suggested that two proteins, Bud8p and Bud9p, might act as bipolar landmarks in diploids (Chant, 1999; Harkins et al., 2001; Taheri et al., 2000). Mutations in these genes affect the bipolar budding pattern in distinct ways whereas the axial pattern of haploids is not affected (Mösch and Fink, 1997; Taheri et al., 2000; Zahner et al., 1996). bud8/bud8 mutants bud at the proximal pole, whereas bud9/bud9 cells bud at the distal pole. bud8/bud9 double mutants produce a random bud site selection pattern in diploid cells (Chant, 1999; Harkins et al., 2001; Taheri et al., 2000).

The subcellular localization of both proteins has been investigated, demonstrating that Bud8p and Bud9p proteins localize at the bud tip of daughter cells and also concentrate at the mother-bud neck (Harkins et al., 2001; Taheri et al., 2000). However, Bud9p was found predominantly at the proximal pole (Harkins et al., 2001). The primary sequences of Bud8p and Bud9p predict related transmembrane proteins with 450-500 amino acid extracellular domains, two membrane spanning domains, short cytoplasmic loops and short extracellular domains. The large Nterminal domains of both proteins are strongly $\mathrm{N}$ - and O-glycosylated (Harkins et al., 2001). However, the topology of both proteins is unknown. The cytoplasmic loops of Bud8p and Bud9p are related in sequence, suggesting that they might recruit components of the common budding factors, perhaps the Rsr1p/Bud2p/Bud5p GTPase signaling module, which appears to transmit the positional information from bipolar cortical markers to the proteins responsible for cell polarization (Chant, 1999; Harkins et al., 2001; Taheri et al., 2000). Recent investigations suggested that the expression of Bud8p and Bud9p is cell cycle regulated, where the timing and site of localization of each protein depends primarily on when in the cell cycle its gene is transcribed (Schenkman et al., 2002). Promoter-swapping experiments demonstrated that expression of Bud8p from BUD9 promoter leads to its localization to the sites typical for Bud9p therefore, it appears to provide Bud9p function and vice versa. When Bud9p is expressed from the BUD8 promoter, it localizes as if it were Bud8p. However, the Bud9p localization signal appears weak, and the protein at the distal pole is only partially effective in providing Bud8p function (Schenkman et al., 2002). The localization of Bud8p appears to depend on actin whereas Bud9p localization is actin- and septindependent (Harkins et al., 2001; Ni and Snyder, 2001; Schenkman et al., 2002). This 
observation was obtained using the actin inhibitor Latrunculin A (Lat A), where patches of GFP-Bud8p signals were undetectable (Harkins et al., 2001; Schenkman et al., 2002). Moreover, Bud8p is not localized in a bnil/bnil actin polarity defect mutant strain, suggesting that formin protein Bnilp might be required for localization or maintenance of bipolar tags at their proper sites (Sheu et al., 2000; (Harkins et al., 2001; Ni and Snyder, 2001). In a large-scale two-hybrid experiment, two proteins were found that interacted with Bud8p. A PAK kinase, Ste20p, and an essential protein of unknown function, Yklo82cp showed interaction with Bud8p (Drees et al., 2001). It has further been proposed that Ste20p may regulate bud site selection by directly phosphorylating the distal tag, Bud8p (Drees et al., 2001; Ni and Snyder, 2001; Sheu et al., 2000).

Recently, it has been hypothesized that another membrane protein, Rax $2 p$, could be part of the bipolar landmark that may form part of the mark at the proximal pole (Chen et al., 2000). RAX2 is predicted to encode a novel type 2 membrane-spanning protein, which is required for bipolar budding pattern. Rax $2 p$ is localized at the mother-bud neck and is then inherited by the daughter cell, which will form a new Rax2p ring at the distal pole in the next cell cycle (Chen et al., 2000).

\subsection{Polarity establishment proteins Rsr1p/Bud1p and Cdc42p}

Rsr1p/Bud1p is a Ras-related GTPase that is thought to help direct bud formation components to the selected site of growth (Madden and Snyder, 1998). Rsr1p/Bud1p activity is regulated by a GTPase-activating protein, Bud2p (Bender, 1993; Park et al., 1993) and a guanine-nucleotide exchange factor, Bud5p (Chant et al., 1991; Powers et al., 1991; Zheng et al., 1995) that form together the Rsr1p/Bud1p GTPase signaling module. How these GTPase-module/polarity-establishment components interact with cortical tags at the selected site is unknown. The Rsr1p/Bud1p GTPase signaling module appears to direct bud formation components to cortical tags at future bud sites by interaction with Cdc42p, Cdc24p, and Bem1p, polarityestablishment components important for bud emergence (Chant et al., 1991; Chant and Herskowitz, 1991; Herskowitz et al., 1995; Michelitch and Chant, 1996; Park et al., 1993). The linking an axial-specific landmark to polarity establishment has been shown by physically interaction between Ax12p/Bud10p and Bud5p (Kang et al., 2001). Whether Bud5p is also involved to link bipolar landmarks Bud8p and Bud9p to cell polarity is not clear. Cdc42p is a Rho-type GTPase (Johnson and Pringle, 1990) whose activity is regulated by the (GEF) Cdc24p (Zheng et al., 1994). Genetic evidence suggests an interaction between the Rsr1p/Bud1p and Cdc42p GTPase modules (Bender, 1993; Bender and Pringle, 1989; Ruggieri et al., 1992). 
GTP-bound Rsr1p/Bud1p binds to Cdc24p and GDP-bound Cdc42p (Park et al., 1997; Zheng et al., 1995), whereas GDP-bound Rsr1p/Bud1p binds Bem1p (Park et al. 1997), another protein involved in the establishment of yeast cell polarity.(Chant et al., 1991; Chenevert et al., 1992). GTPase activation plays a role in targeting or assembling bud formation components at the incipient bud site and may help to initiate the bud formation process (Michelitch and Chant, 1996; Park et al., 1997; Zheng et al., 1995).

\section{5. Polarization of the actin cytoskeleton by formins and PAKs}

Bnilp and Bnrlp formins are involved in cell polarity processes and polarization of the actin cytoskeleton. Bnilp binds to Cdc42p and its effectors, Ste20p and Cla4p, to link Rho-GTPase signaling to actin filament assembly (Evangelista et al., 1997). The protein kinases Ste20p and Cla4p are essential to Cdc42p-actin signaling at all stages of growth, and simultaneous loss of Ste20p and Cla4p blocks initial bud emergence, bud growth and cytokinesis (Cvrckova et al., 1995; Eby et al., 1998; Holly and Blumer, 1999; Richman et al., 1999). The type I myosins (Myo3p and Myo5p) are the only cytoskeletal substrates of PAKs identified so far in yeast (Wu et al., 1997). These motor proteins, which localize to cortical patches, are necessary for organization of actin cytoskeleton (Evangelista et al., 2000; Geli and Riezman, 1996; Goodson et al., 1996; Lechler et al., 2000). The PAK-mediated phosphorylation by Ste20p and Cla4p is essential for mysonis I activity (Wu et al., 1997). A key regulator of actin assembly is the actin-related-protein (Arp2/3) complex which is regulated by Bee1p functioning with the type I myosins Myo3p and Myo5p (Evangelista et al., 2000; Lechler et al., 2001; Lechler et al., 2000; Winter et al., 1999). Active Cdc42p recruits the Bee1p-Vrp1p-Arp2/3 complex and type I myosins to the polarization site in a formin-dependent process (Lechler et al., 2001). Recruitment of this complex is thought to mark the site of actin polymerization and to initiate budding at the selected site.

\subsection{Polarized secretion along actin cables}

New plasma membrane and cell wall material must be directed to the new growth site, presumably through the secretory pathway to mediate bud formation and growth. Polarized growth at the yeast cell surface depends upon delivery of secretory vesicles along actin cables by the type $\mathrm{V}$ myosin Myo2p, and tropomyosin, an actin filament-binding protein (Finger and Novick, 1998; Pruyne and Bretscher, 2000b). Tropomyosin mutations (tpml 1 tmp2 2 ) result in a disassembly of actin cables (Pruyne and Bretscher, 2000b). The transport of vesicles from Golgi and 
endosomal elements from the mother into the bud leads to accumulation at the cap (Pruyne and Bretscher, 2000b). Myo2p-driven vesicle transport along actin cables is fast. The C-terminal tail of Myo2p associates with vesicles. Three proteins Smy1p, $\operatorname{Sec} 2 \mathrm{p}$ and Sec4p are involved in assembling Myo2p-vesicle complexes, and their functions are essential for vesicle delivery in the mother cell to the cap at the bud tip along actin cables (Govindan et al., 1995; Lillie and Brown, 1992; Pruyne and Bretscher, 2000b; Schott et al., 1999; Walch-Solimena et al., 1997). Sec4p is a Rab GTPase that is essential for fusion of secretory vesicles with the plasma membrane. $\operatorname{Sec} 2 \mathrm{p}$ is the nuleotide-exchange factor for Sec4p and required for delivery of vesicles. Sec2p or GTP-Sec4p on vesicles promotes Myo2p-Smy1p binding (WalchSolimena et al., 1997), and this vesicle complex is polarized along actin cables to the cap at the bud tip that leads to isotropic bud growth (Pruyne and Bretscher, 2000a; Pruyne and Bretscher, 2000b). In yeast, exocytosis is an actin-independent process for polarized vesicle transport that requires plasma membrane t-SNAREs, the t-SNARE-interacting protein Sec1p, and the exocyst complex (Finger and Novick, 1998). The actin-dependent transport of vesicles is coupled to the actinindependent establishment of a site for vesicle docking and fusion. First activated Sec4p (by Sec2p) binds to vesicles with appropriate v-SNAREs from Golgi and allows polarized delivery of post-Golgi vesicles by Myo2 $p$ along actin cables. The $t-$ SNAREs are distributed over the entire plasma membrane, but Sec3p is associated with the plasma membrane specifically at exocytic sites (docking site). The v- and tSNAREs could then interact, promoting fusion of the vesicle with the plasma membrane (Finger and Novick, 1998; Pruyne and Bretscher, 2000b).

\subsection{Polarized mRNA transport}

The transport of messenger RNA along actin cables is another polarization event in yeast. Cytoplasmic transport of most localized mRNAs requires a functional cytoskeleton and motor proteins (Jansen, 1999 ; Jansen, 2001). The first step is the recognition of cytoplasmic components of the localized mRNA by mRNA-binding proteins. After mRNA recognition, the associated RNP (ribonucleoprotein) complex binds the motor protein and is transported to its destination. Finally, mRNA anchors at cortical protein and mediates the translation of the target protein (Figure 6). ASH1 encodes a daughter-specific nuclear repressor of $H O$ transcription (Jansen et al., 1996; Sil and Herskowitz, 1996). Asymmetrical distribution of Ash1p is mediated by localization of ASH1 mRNA in a cap at the distal pole of daughter cells (Long et al., 1997; Takizawa et al., 1997). Polarized localization of ASH1 mRNA requires actin and actin-binding proteins such as Bnilp (Nasmyth and Jansen, 1997). 


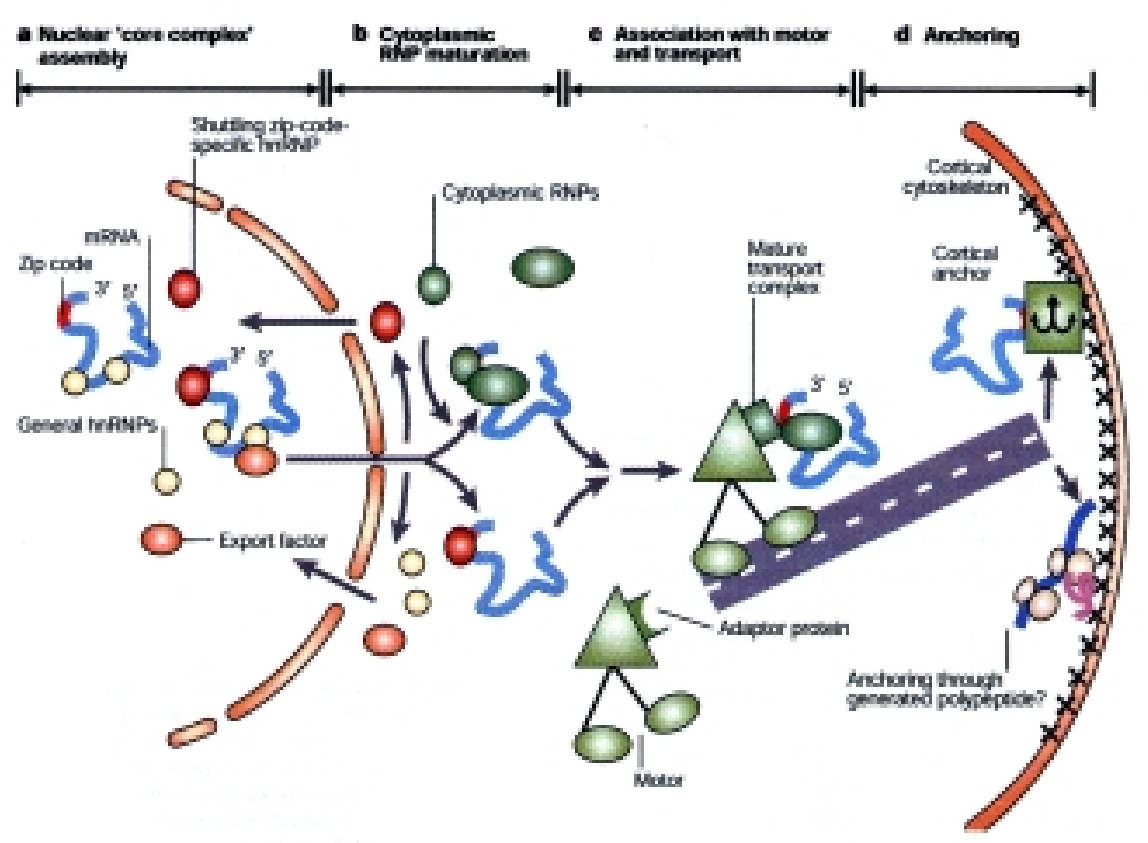

Figure 7. Model for mRNA transport. a) Core complex assembly. Heterologous nuclear ribonucleoproteins, hnRNPs bind to mRNA (blue) inside the nucleus. The general hnRNPs (yellow circle) and specific hnRNPs (red ovals) recognize the localization signal (zip code, red) of the mRNA. The localized mRNAs assemble with mRNA export factor (orange oval), and the mRNARNP complex is exported to the cytoplasm. b) Cytoplasmic RNP maturation. General hnRNPs and export factors go back to the nucleus, whereas specific hnRNPs stay associated with the mRNA. Specific hnRNPs could be released from the transcript and replaced by cytoplasmic zip-code-specific RNPs (green ovals). c) Association with motor protein and transport. The mature RNP complex associates with a motor protein (green triangle) and is transported to the target site using adapter proteins. The RNP associates with membranous structures (for example, endoplasmic reticulum) and is transported by piggyback. d) Anchoring. Finally, the RNP is released from the motor and is tethered to the target site by specific proteins (cortical anchor) or through a translation-dependent process. Translation is allowed only at the target site.

In addition, several RNA-binding proteins, Mpt5p, Scp160p and Khd1p are required for $A S H 1$ mRNA localization. ASH 1 mRNA is partially delocalized in $m p t 5 \Delta$, scp $160 \Delta$ and $k h d 1 \Delta$ mutants (Irie et al., 2002). These proteins may function in the linkage between ASH1 mRNA localization and its translation.

Whether landmark components such as Bud8p and Bud9p are involved in the transport and asymmetrical localization of $A S H 1$ mRNA is not known. 


\section{Aim of this work}

The aim of this work was the functional analysis of the two putative landmark proteins Bud8p and Bud9p in the budding yeast Saccharomyces cerevisiae. Specifically, the following goals should be achieved:

- The subcellular localization of Bud8p, and Bud9p were to be analysed by fusion to the green fluorescent protein (GFP) and observation in living cells. In addition, localization should be investigated by indirect immunofluorescence using epitopetagged versions of Bud8p and Bud9p.

- Regulation of Bud8p and Bud9p by nitrogen starvation should be analysed. Therefore, the mRNA expression levels of BUD8 and BUD9 genes as well as intracellular levels of Bud8p and Bud9p proteins and their subcellular localization should be investigated in single yeast form (YF) cells a in cells of pseudohyphal (PH) filaments.

- In order to identify novel functional domains of Bud8p, a systematic deletion analysis of the BUD8 gene should be performed followed by a functional analysis of the distinct bud8 deletion mutations.

- Putative downstream-acting factors of both Bud8p and Bud9p should be identified by genetic and biochemical assays. In a first step, genetical and physical interactions of Bud8p and Bud9p with known polarity-establishment proteins and actin associated-proteins should be tested by double mutant analysis and co-purification experiments. In a second step, novel interaction-partners should be identified by using the yeast two-hybrid system, followed by biochemical characterization of interactions. 


\section{References}

Adams, A.E. and Pringle, J.R. (1984) Relationship of actin and tubulin distribution to bud growth in wild- type and morphogenetic-mutant Saccharomyces cerevisiae. J. Cell Biol., 98, 934-945.

Amberg, D.C., Zahner, J.E., Mulholland, J.W., Pringle, J.R. and Botstein, D. (1997) Aip3p/Bud6p, a yeast actin-interacting protein that is involved in morphogenesis and the selection of bipolar budding sites. Mol. Biol. Cell., 8, 729-753.

Bähler, J. and Peter, M. (2000) Cell polarity in yeast. In Drubin, D.G. (ed.) Cell polarity. Oxford University Press, Oxford, Vol. 28, pp. 21-77.

Bender, A. (1993) Genetic evidence for the roles of the bud-site-selection genes BUD5 and BUD2 in control of the Rsr1p (Bud1p) GTPase in yeast. Proc. Natl. Acad. Sci. USA, 90, 9926-9929.

Bender, A. and Pringle, J.R. (1989) Multicopy suppression of the $c d c 24$ budding defect in yeast by $C D C 42$ and three newly identified genes including the rasrelated gene RSR1. Proc. Natl. Acad. Sci. USA, 86, 9976-9980.

Burda, P. and Aebi, M. (1998) The ALG10 locus of Saccharomyces cerevisiae encodes the alpha-1,2 glucosyltransferase of the endoplasmic reticulum: the terminal glucose of the lipid-linked oligosaccharide is required for efficient $\mathrm{N}$ linked glycosylation. Glycobiology, 8, 455-462.

Casamayor, A. and Snyder, M. (2002) Bud-site selection and cell polarity in budding yeast. Curr. Opin. Microbiol., 5, 179-186.

Chant, J. (1999) Cell polarity in yeast. Annu. Rev. Cell Dev. Biol., 15, 365-391.

Chant, J., Corrado, K., Pringle, J.R. and Herskowitz, I. (1991) Yeast BUD5, encoding a putative GDP-GTP exchange factor, is necessary for bud site selection and interacts with bud formation gene BEM1. Cell, 65, 1213-1224.

Chant, J. and Herskowitz, I. (1991) Genetic control of bud site selection in yeast by a set of gene products that constitute a morphogenetic pathway. Cell, 65, 12031212.

Chant, J., Mischke, M., Mitchell, E., Herskowitz, I. and Pringle, J.R. (1995) Role of Bud3p in producing the axial budding pattern of yeast. J. Cell Biol., 129, 767778.

Chant, J. and Pringle, J.R. (1995) Patterns of bud-site selection in the yeast Saccharomyces cerevisiae. J. Cell Biol., 129, 751-765.

Chen, T., Hiroko, T., Chaudhuri, A., Inose, F., Lord, M., Tanaka, S., Chant, J. and Fujita, A. (2000) Multigenerational cortical inheritance of the Rax2 protein in orienting polarity and division in yeast. Science, 290, 1975-1978. 
Chenevert, J., Corrado, K., Bender, A., Pringle, J. and Herskowitz, I. (1992) A yeast gene $(B E M 1)$ necessary for cell polarization whose product contains two $\mathrm{SH} 3$ domains. Nature, 356, 77-79.

Costigan, C. and Snyder, M. (1994) SLK1, a yeast homolog of MAP kinase activators, has a RAS/cAMP-independent role in nutrient sensing. Mol. Gen. Genet., 243, 286-296.

Cvrckova, F., De Virgilio, C., Manser, E., Pringle, J.R. and Nasmyth, K. (1995) Ste20-like protein kinases are required for normal localization of cell growth and for cytokinesis in budding yeast. Genes Dev., 9, 1817-1830.

Dix, I., Russell, C., Yehuda, S.B., Kupiec, M. and Beggs, J.D. (1999) The identification and characterization of a novel splicing protein, Isylp, of Saccharomyces cerevisiae. RNA, 5, 360-368.

Drees, B.L., Sundin, B., Brazeau, E., Caviston, J.P., Chen, G.C., Guo, W., Kozminski, K.G., Lau, M.W., Moskow, J.J., Tong, A., Schenkman, L.R., McKenzie, A., 3rd, Brennwald, P., Longtine, M., Bi, E., Chan, C., Novick, P., Boone, C., Pringle, J.R., Davis, T.N., Fields, S. and Drubin, D.G. (2001) A protein interaction map for cell polarity development. J. Cell Biol., 154, 549-571.

Drubin, D.G. and Nelson, W.J. (1996) Origins of cell polarity. Cell, 84, 335-344.

Duncan, M.C., Cope, M.J., Goode, B.L., Wendland, B. and Drubin, D.G. (2001) Yeast Eps15-like endocytic protein, Pan1p, activates the Arp2/3 complex. Nat. Cell Biol., 3, 687-690.

Eby, J.J., Holly, S.P., van Drogen, F., Grishin, A.V., Peter, M., Drubin, D.G. and Blumer, K.J. (1998) Actin cytoskeleton organization regulated by the PAK family of protein kinases. Curr. Biol., 8, 967-970.

Evangelista, M., Blundell, K., Longtine, M.S., Chow, C.J., Adames, N., Pringle, J.R., Peter, M. and Boone, C. (1997) Bnilp, a yeast formin linking Cdc42p and the actin cytoskeleton during polarized morphogenesis. Science, 276, 118-122.

Evangelista, M., Klebl, B.M., Tong, A.H., Webb, B.A., Leeuw, T., Leberer, E., Whiteway, M., Thomas, D.Y. and Boone, C. (2000) A role for myosin-I in actin assembly through interactions with Vrp1p, Bee1p, and the Arp2/3 complex. $J$. Cell Biol., 148, 353-362.

Finger, F.P. and Novick, P. (1998) Spatial regulation of exocytosis: lessons from yeast. J. Cell Biol., 142, 609-612.

Flescher, E.G., Madden, K. and Snyder, M. (1993) Components required for cytokinesis are important for bud site selection in yeast. J. Cell Biol., 122, 373386.

Fujita, A., Oka, C., Arikawa, Y., Katagai, T., Tonouchi, A., Kuhara, S. and Misumi, Y. (1994) A yeast gene necessary for bud-site selection encodes a protein similar to insulin-degrading enzymes. Nature, 372, 567-570. 
Fujiwara, T., Tanaka, K., Mino, A., Kikyo, M., Takahashi, K., Shimizu, K. and Takai, Y. (1998) Rho1p-Bnilp-Spa2p interactions: implication in localization of Bnilp at the bud site and regulation of the actin cytoskeleton in Saccharomyces cerevisiae. Mol. Biol. Cell, 9, 1221-1233.

Gehrung, S. and Snyder, M. (1990) The SPA2 gene of Saccharomyces cerevisiae is important for pheromone-induced morphogenesis and efficient mating. J. Cell Biol., 111, 1451-1464.

Geli, M.I. and Riezman, H. (1996) Role of type I myosins in receptor-mediated endocytosis in yeast. Science, 272, 533-535.

Gimeno, C.J., Ljungdahl, P.O., Styles, C.A. and Fink, G.R. (1992) Unipolar cell divisions in the yeast $S$. cerevisiae lead to filamentous growth: regulation by starvation and RAS. Cell, 68, 1077-1090.

Goode, B.L. and Rodal, A.A. (2001) Modular complexes that regulate actin assembly in budding yeast. Curr. Opin. Microbiol., 4, 703-712.

Goode, B.L., Rodal, A.A., Barnes, G. and Drubin, D.G. (2001) Activation of the Arp2/3 complex by the actin filament binding protein Abp1p. J. Cell Biol., 153, 627-634.

Goodson, H.V., Anderson, B.L., Warrick, H.M., Pon, L.A. and Spudich, J.A. (1996) Synthetic lethality screen identifies a novel yeast myosin I gene (MYO5): myosin I proteins are required for polarization of the actin cytoskeleton. J. Cell Biol., 133, 1277-1291.

Govindan, B., Bowser, R. and Novick, P. (1995) The role of Myo2, a yeast class V myosin, in vesicular transport. J. Cell Biol., 128, 1055-1068.

Gow, N.A.R. (1996) TIP GROWTH AND POLARITY. In Gow, N.A.R.a.G., Geoffrey M. (ed.) THE GROWING FUNGUS. Chapman \& Hall, London, London, pp. 277-299.

Halme, A., Michelitch, M., Mitchell, E.L. and Chant, J. (1996) Bud10p directs axial cell polarization in budding yeast and resembles a transmembrane receptor. Curr. Biol., 6, 570-579.

Harkins, H.A., Page, N., Schenkman, L.R., De Virgilio, C., Shaw, S., Bussey, H. and Pringle, J.R. (2001) Bud8p and Bud9p, proteins that may mark the sites for bipolar budding in yeast. Mol. Biol. Cell, 12, 2497-2518.

Heesen, S., Lehle, L., Weissmann, A. and Aebi, M. (1994) Isolation of the ALG5 locus encoding the UDP-glucose:dolichyl-phosphate glucosyltransferase from Saccharomyces cerevisiae. Eur. J. Biochem., 224, 71-79.

Herskowitz, I. (1995) MAP kinase pathways in yeast: for mating and more. Cell, 80, 187-197. 
Herskowitz, I., Park, H.O., Sanders, S., Valtz, N. and Peter, M. (1995) Programming of cell polarity in budding yeast by endogenous and exogenous signals. Cold Spring. Harb. Symp. Quant. Biol., 60, 717-727.

Hofmeister, A. and Brun, Y.V. (2000) Polarity and cell fate in bacteria. In Drubin, D.G. (ed.) Cell polarity. Oxford University Press, Oxford, Vol. 28, pp. 1-20.

Holly, S.P. and Blumer, K.J. (1999) PAK-Family Kinases Regulate Cell and Actin Polarization throughout the Cell Cycle of Saccharomyces cerevisiae. J. Cell Biol., 147, 845-856.

Irie, K., Tadauchi, T., Takizawa, P.A., Vale, R.D., Matsumoto, K. and Herskowitz, I. (2002) The Khd1 protein, which has three KH RNA-binding motifs, is required for proper localization of ASH1 mRNA in yeast. EMBO J., 21, 1158-1167.

Jansen, R.-P., Dowzer, C., Michaelis, C., Galova, M. and Nasmyth, K. (1996) Mother cell-specific $H O$ expression in budding yeast depends on the unconventional Myo4p and other cytoplasmic proteins. Cell, 84, 687-697.

Jansen, R.P. (1999) RNA-cytoskeletal associations. FASEB J., 13, 455-466.

Jansen, R.P. (2001) mRNA localization: message on the move. Nat. Rev. Mol. Cell Biol., 2, 247-256.

Jeng, R.L. and Welch, M.D. (2001) Cytoskeleton: actin and endocytosis--no longer the weakest link. Curr. Biol., 11, R691-694.

Jin, H. and Amberg, D.C. (2000) The secretory pathway mediates localization of the cell polarity regulator Aip3p/Bud6p. Mol. Biol. Cell, 11, 647-661.

Johnson, D.I. and Pringle, J.R. (1990) Molecular characterization of CDC42, a Saccharomyces cerevisiae gene involved in the development of cell polarity. $J$. Cell Biol., 111, 143-152.

Kang, P.J., Sanson, A., Lee, B. and Park, H.O. (2001) A GDP/GTP exchange factor involved in linking a spatial landmark to cell polarity. Science, 292, 1376-1378.

Kohno, H., Tanaka, K., Mino, A., Umikawa, M., Imamura, H., Fujiwara, T., Fujita, Y., Hotta, K., Qadota, H., Watanabe, T., Ohya, Y. and Takai, Y. (1996) Bnilp implicated in cytoskeletal control is a putative target of Rholp small GTP binding protein in Saccharomyces cerevisiae. EMBO J., 15, 6060-6068.

Kron, S.J., Styles, C.A. and Fink, G.R. (1994) Symmetric cell division in pseudohyphae of the yeast Saccharomyces cerevisiae. Mol. Biol. Cell, 5, 10031022.

Lechler, T., Jonsdottir, G.A., Klee, S.K., Pellman, D. and Li, R. (2001) A two-tiered mechanism by which Cdc42 controls the localization and activation of an Arp2/3activating motor complex in yeast. J. Cell Biol., 155, 261-270. 
Lechler, T., Shevchenko, A. and Li, R. (2000) Direct involvement of yeast type I myosins in Cdc42-dependent actin polymerization. J. Cell Biol., 148, 363-373.

Lew, D.J. and Reed, S.I. (1993) Morphogenesis in the yeast cell cycle: regulation by Cdc28 and cyclins. J. Cell Biol., 120, 1305-1320.

Lew, D.J. and Reed, S.I. (1995) Cell cycle control of morphogenesis in budding yeast. Curr. Opin. Genet. Dev., 5, 17-23.

Lillie, S.H. and Brown, S.S. (1992) Suppression of a myosin defect by a kinesinrelated gene. Nature, 356, 358-361.

Long, R.M., Singer, R.H., Meng, X., Gonzalez, I., Nasmyth, K. and Jansen, R.P. (1997) Mating type switching in yeast controlled by asymmetric localization of ASH1 mRNA. Science, 277, 383-387.

Longtine, M.S., De Marini, D.J., Valencik, M.L., Al-Awar, O.S., Fares, H., Del Virgilio, C. and Pringle, J.R. (1996) The septins: roles in cytokinesis and other processes. Curr. Opin. Cell Biol., 8, 106-119.

Madden, K. and Snyder, M. (1998) Cell polarity and morphogenesis in budding yeast. Annu. Rev. Microbiol., 52, 687-744.

Michelitch, M. and Chant, J. (1996) A mechanism of Bud1p GTPase action suggested by mutational analysis and immunolocalization. Curr. Biol., 6, 446454.

Mösch, H.-U. and Fink, G.R. (1997) Dissection of filamentous growth by transposon mutagenesis in Saccharomyces cerevisiae. Genetics, 145, 671-684.

Munn, A.L. (2001) Molecular requirements for the internalisation step of endocytosis: insights from yeast. Biochim. Biophys. Acta., 1535, 236-257.

Nasmyth, K. and Jansen, R.P. (1997) The cytoskeleton in mRNA localization and cell differentiation. Curr. Opin. Cell Biol., 9, 396-400.

Ni, L. and Snyder, M. (2001) A Genomic Study of the Bipolar Bud Site Selection Pattern in Saccharomyces cerevisiae. Mol. Biol. Cell, 12, 2147-2170.

Ozaki-Kuroda, K., Yamamoto, Y., Nohara, H., Kinoshita, M., Fujiwara, T., Irie, K. and Takai, Y. (2001) Dynamic localization and function of Bnilp at the sites of directed growth in Saccharomyces cerevisiae. Mol. Cell Biol., 21, 827-839.

Park, H.O., Bi, E., Pringle, J.R. and Herskowitz, I. (1997) Two active states of the Ras-related Bud1/Rsr1 protein bind to different effectors to determine yeast cell polarity. Proc. Natl. Acad. Sci. USA, 94, 4463-4468.

Park, H.O., Chant, J. and Herskowitz, I. (1993) BUD2 encodes a GTPase-activating protein for Bud1/Rsr1 necessary for proper bud-site selection in yeast. Nature, 365, 269-274. 
Powers, S., Gonzales, E., Christensen, T., Cubert, J. and Broek, D. (1991) Functional cloning of BUD5, a CDC25-related gene from S. cerevisiae that can suppress a dominant-negative RAS2 mutant. Cell, 65, 1225-1231.

Pringle, J.R., Bi, E., Harkins, H.A., Zahner, J.E., De Virgilio, C., Chant, J., Corrado, K. and Fares, H. (1995) Establishment of cell polarity in yeast. Cold Spring. Harb. Symp. Quant. Biol., 60, 729-744.

Pruyne, D. and Bretscher, A. (2000a) Polarization of cell growth in yeast. I. Establishment and maintenance of polarity states. J. Cell Sci., 113, 365-375.

Pruyne, D. and Bretscher, A. (2000b) Polarization of cell growth in yeast. II. The role of the cortical actin cytoskeleton. J. Cell Sci., 113, 571-585.

Reiss, G., te Heesen, S., Zimmerman, J., Robbins, P.W. and Aebi, M. (1996) Isolation of the ALG6 locus of Saccharomyces cerevisiae required for glucosylation in the N-linked glycosylation pathway. Glycobiology, 6, 493-498.

Richman, T.J., Sawyer, M.M. and Johnson, D.I. (1999) The Cdc42p GTPase is involved in a $\mathrm{G} 2 / \mathrm{M}$ morphogenetic checkpoint regulating the apical-isotropic switch and nuclear division in yeast. J. Biol. Chem., 274, 16861-16870.

Roemer, T., Madden, K., Chang, J. and Snyder, M. (1996) Selection of axial growth sites in yeast requires Axl2p, a novel plasma membrane glycoprotein. Genes Dev., 10, 777-793.

Rossanese, O.W., Reinke, C.A., Bevis, B.J., Hammond, A.T., Sears, I.B., O'Connor, J. and Glick, B.S. (2001) A role for actin, Cdc1p, and Myo2p in the inheritance of late Golgi elements in Saccharomyces cerevisiae. J. Cell Biol., 153, 47-62.

Ruggieri, R., Bender, A., Matsui, Y., Powers, S., Takai, Y., Pringle, J.R. and Matsumoto, K. (1992) RSR 1, a ras-like gene homologous to Krev-1 (smg21A/rap 1A): role in the development of cell polarity and interactions with the Ras pathway in Saccharomyces cerevisiae. Mol. Cell. Biol., 12, 758-766.

Sanders, S.L., Gentzsch, M., Tanner, W. and Herskowitz, I. (1999) O-Glycosylation of Ax12/Bud10p by Pmt $4 \mathrm{p}$ is required for its stability, localization, and function in daughter cells. J. Cell Biol., 145, 1177-1188.

Sanders, S.L. and Herskowitz, I. (1996) The BUD4 protein of yeast, required for axial budding, is localized to the mother/BUD neck in a cell cycle-dependent manner. J. Cell Biol., 134, 413-427.

Schenkman, L.R., Caruso, C., Page, N. and Pringle, J.R. (2002) The role of cell cycle-regulated expression in the localization of spatial landmark proteins in yeast. J. Cell Biol., 156, 829-841.

Schott, D., Ho, J., Pruyne, D. and Bretscher, A. (1999) The COOH-terminal domain of Myo2p, a yeast myosin $\mathrm{V}$, has a direct role in secretory vesicle targeting. $J$. Cell Biol., 147, 791-808. 
Sheu, Y.J., Barral, Y. and Snyder, M. (2000) Polarized growth controls cell shape and bipolar bud site selection in Saccharomyces cerevisiae. Mol. Cell. Biol., 20, 5235-5247.

Sheu, Y.J., Santos, B., Fortin, N., Costigan, C. and Snyder, M. (1998) Spa2p interacts with cell polarity proteins and signaling components involved in yeast cell morphogenesis. Mol. Cell. Biol., 18, 4053-4069.

Sil, A. and Herskowitz, I. (1996) Identification of asymmetrically localized determinant, Ash1p, required for lineage-specific transcription of the yeast $\mathrm{HO}$ gene. Cell, 84, 711-722.

Snyder, M. (1989) The Spa2p protein of yeast localizes to sites of cell growth. $J$. Cell Biol., 108, 1419-1429.

Snyder, M., Gehrung, S. and Page, B.D. (1991) Studies concerning the temporal and genetic control of cell polarity in Saccharomyces cerevisiae. J. Cell Biol., 114, 515-532.

Stagljar, I., te Heesen, S. and Aebi, M. (1994) New phenotype of mutations deficient in glucosylation of the lipid-linked oligosaccharide: cloning of the ALG8 locus. Proc. Natl. Acad. Sci. U S A, 91, 5977-5981.

Taheri, N., Köhler, T., Braus, G.H. and Mösch, H.-U. (2000) Asymmetrically localized Bud8p and Bud9p proteins control yeast cell polarity and development. EMBO J., 19, 6686-6696.

Takizawa, P.A., Sil, A., Swedlow, J.R., Herskowitz, I. and Vale, R.D. (1997) Actindependent localization of an RNA encoding a cell-fate determinant in yeast. Nature, 389, 90-93.

Tharun, S., He, W., Mayes, A.E., Lennertz, P., Beggs, J.D. and Parker, R. (2000) Yeast Sm-like proteins function in mRNA decapping and decay. Nature, 404, 515-518.

Valtz, N. and Herskowitz, I. (1996) Pea2 protein of yeast is localized to sites of polarized growth and is required for efficient mating and bipolar budding. J. Cell Biol., 135, 725-739.

Walch-Solimena, C., Collins, R.N. and Novick, P.J. (1997) Sec2p mediates nucleotide exchange on Sec4p and is involved in polarized delivery of post-Golgi vesicles. J. Cell Biol., 137, 1495-1509.

Winter, D., Lechler, T. and Li, R. (1999) Activation of the yeast Arp2/3 complex by Bee1p, a WASP-family protein. Curr. Biol., 9, 501-504.

Wu, C., Lytvyn, V., Thomas, D.Y. and Leberer, E. (1997) The phosphorylation site for Ste20p-like protein kinases is essential for the function of myosin-I in yeast. J. Biol. Chem., 272, 30623-30626. 
Zahner, J.E., Harkins, H.A. and Pringle, J.R. (1996) Genetic analysis of the bipolar pattern of bud site selection in the yeast Saccharomyces cerevisiae. Mol. Cell. Biol., 16, 1857-1870.

Zheng, Y., Bender, A. and Cerione, R.A. (1995) Interactions among proteins involved in bud-site selection and bud-site assembly in Saccharomyces cerevisiae. J. Biol. Chem., 270, 626-630.

Zheng, Y., Cerione, R. and Bender, A. (1994) Control of the yeast bud-site assembly GTPase Cdc42. Catalysis of guanine nucleotide exchange by Cdc24 and stimulation of GTPase activity by Bem3. J. Biol. Chem., 269, 2369-2372. 


\title{
Chapter 2
}

\section{Asymmetrically localized Bud8p and Bud9p proteins control yeast cell polarity and development}

\begin{abstract}
Diploid strains of the budding yeast Saccharomyces cerevisiae change the pattern of cell division from bipolar to unipolar when switching growth from the unicellular yeast-form (YF) to filamentous, pseudohyphal $(\mathrm{PH})$ cells in response to nitrogen starvation. The functions of two transmembrane proteins, Bud8p and Bud9p, in regulating YF and PH cell polarity were investigated. Bud8p is highly concentrated at the distal pole of both YF and PH cells, where it directs initiation of cell division. Asymmetric localization of Bud8p is independent of the Rsr1p/Bud1p GTPase. rsrl/budl mutations are epistatic to bud8 mutations, placing Rsr1p/Bud1p downstream of Bud8p. In YF cells, Bud9p is also localized at the distal pole, yet deletion of BUD9 favors distal bud initiation. In PH cells, nutritional starvation for nitrogen efficiently prevents distal localization of Bud9p. Because Bud8p and Bud9p proteins associate in vivo, we propose Bud8p as landmark for bud initiation at the distal cell pole, where Bud9p acts as inhibitor. In response to nitrogen starvation, asymmetric localization of Bud9p is averted, favoring Bud8p-mediated cell division at the distal pole.
\end{abstract}




\section{Introduction}

Control of cell polarity is fundamental for the development of many organisms. The yeast Saccharomyces cerevisiae is a simple model for studying the molecular basis underlying establishment of cell polarity and oriented cell division. These yeast cells divide by budding and choose cell division sites in different spatial patterns that are under genetic control of their cell type (Freifelder, 1960; Hicks et al., 1977; Chant and Pringle, 1995). Haploid a or $\alpha$ cells bud in an axial pattern, where mother and daughter cells bud adjacent to their cell pole that defined the previous motherdaughter junction. This region of the yeast cell surface is also referred to as the proximal pole or the birth end of the cell. Diploid $\mathbf{a} / \alpha$ yeast cells bud in a bipolar pattern, where buds form either at the proximal pole or at the site opposite to it, called the distal pole.

Yeast cell polarity and according budding patterns are affected by extracellular stimuli, such as pheromones or nutrients. For instance, haploid cells that have been exposed to a concentration gradient of pheromone of the opposite mating partner redirect their axis of polarity and start to form mating projections in the direction of the presumed mating partner (Segall, 1993). In addition, budding patterns of haploid cells can be altered by nutritional starvation (Madden and Snyder, 1992; Chant and Pringle, 1995). Diploid cells starved for nitrogen switch their budding pattern from bipolar to unipolar distal, where most of the buds emerge at the distal cell pole (Gimeno et al., 1992; Kron et al., 1994). The unipolar distal budding program is essential for the formation of multicellular filaments called pseudohyphae $(\mathrm{PH})$, whose development is induced when diploid cells are starved for nitrogen and subsequently change cell morphology and show substrate invasive growth behavior. Unipolar distal budding is a prerequisite for the establishment of filamentous structures and therefore can be viewed as a process regulated by nutritional signals and guiding the direction of the growing PH filaments.

In yeast, selection of cell division sites is regulated by at least three different classes of genes and corresponding proteins (for recent reviews see Madden and Snyder, 1998; Chant, 1999). One class of genes is required for axial and bipolar budding and includes RSR1/BUD1, BUD2 and BUD5 (Bender and Pringle, 1989; Chant et al., 1991; Chant and Herskowitz, 1991). Mutations in these genes cause random budding patterns in haploid and diploid yeast form (YF) cells. Rsr1p/Bud1p, Bud2p and Bud5p constitute a GTPase signaling module that is thought to help to direct bud formation components to the selected cell division site (Park et al., 1993; Park et al., 1997). A second class of genes is required specifically for axial budding of haploids 
without affecting the bipolar pattern of diploids. Genes of this class include $A X L 1$, BUD10/AXL2, BUD3 and BUD4 (Chant and Herskowitz, 1991; Fujita et al., 1994; Halme et al., 1996; Roemer et al., 1996). A third class of genes is required for the bipolar budding pattern of diploid yeast cells but not for haploid axial budding. Many genes of this class have been identified by genetic screens and include AIP3/BUD6, BUD7, BUD8, BUD9, BNI1, PEA2 and SPA2 (Snyder, 1989; Valtz and Herskowitz, 1996; Zahner et al., 1996). Mutations in most of these genes cause a random budding pattern only in diploids without affecting axial budding in haploids. Only two genes of this class, $B U D 8$ and $B U D 9$ have been described to shift the bipolar pattern to a unipolar pattern and therefore appear to have the most specific effects on bipolar budding. Mutations in BUD8 cause a unipolar proximal budding pattern in diploids, whereas bud9 mutants bud with high frequency from the distal cell pole (Zahner et al., 1996). Therefore, Bud8p and Bud9p have been proposed to act as bipolar landmarks that might recruit components of the common budding factors, e.g. Bud2p, Bud5p or Rsr1p/Bud1p, to either of the two cell poles (Chant, 1999).

Most studies that have addressed the function of genes controlling bud site selection were performed under nutrient rich conditions, where $S$. cerevisiae will grow and divide in the unicellular yeast form (YF). Only little is known about the molecular mechanisms that control changes in cell polarity in response to nutritional starvation. Because nitrogen starvation causes a switch in the budding pattern from bipolar to unipolar distal in diploid cells, pseudohyphal development is an ideal model to study factors that control oriented cell division in response to external signals. To date, no class of genes has been identified that is specifically required for the unipolar distal pattern of PH cells without affecting bipolar budding of YF cells. An initial study has identified Rsr1p/Bud1p to be required for pseudohyphal development, because expression of a dominant negative form of $R S R 1 / B U D 1, R S R 1^{\text {Asn } 16}$, suppresses filament formation in response to nitrogen starvation (Gimeno et al., 1992). A genetic screen directed at the identification of genes specifically required for pseudohyphal development has uncovered several of the bipolar specific bud site selection genes including BUD8, BNI1, PEA2/DFG9 and SPA2 (Mösch and Fink, 1997).

In this study, we investigated the requirement and subcellular localization of Bud8p and Bud9p proteins during both $\mathrm{YF}$ growth in nutrient rich media and $\mathrm{PH}$ filamentous growth under nitrogen starvation conditions. Our study suggests that Bud8p acts as a landmark for bud initiation at the distal cell pole, whereas Bud9p appears to be an inhibitor of distal budding that might interfere with Bud8p functions 
in YF cells. In PH cells, Bud9p is prevented from being localized to the distal cell pole, causing a switch in cell polarity from bipolar to unipolar budding.

\section{Results}

\section{Bud8p and Bud9p are asymmetrically localized at the distal pole of YF cells}

Previous genetic studies suggested that Bud8p and Bud9p might act as landmarks for the selection of cell division sites. Therefore, we first determined the subcellular localization of Bud8p and Bud9p in diploid cells. GFP-BUD8 and GFP-BUD9 fusion genes expressing GFP-Bud8p and GFP-Bud9p fusion proteins from the endogenous $B U D 8$ - and $B U D 9$-promoters were constructed and expressed in YF cells from low copy plasmids. Low levels of neither GFP-Bud8p nor GFP-Bud9p produced fluorescent signals that were detectable by GFP-fluorescence microscopy, although the highly fluorescent GFPuv (cycle 3) variant was used (Crameri et al., 1996). Importantly, low copy expression of GFP-fusion genes complemented the budding defects of diploid bud8 or bud9 mutant strains, demonstrating that GFPfusion proteins were produced at levels sufficient for function but not for visual detection. GFP-fusion proteins were detectable when GFP-Bud8p and GFP-Bud9p were expressed from high copy plasmids. Localization was first analyzed in exponentially growing cultures in nutrient rich media, when strains grow predominantly in the YF and elaborate the bipolar budding pattern (Figure 8A). GFPBud8p was found to be localized at both the tip of the growing daughter cell and the mother side of the mother-daughter neck. The concentration of GFP-Bud8p was more pronounced at the mother-bud neck than at the bud tip of small-budded YF cells. However, this difference was no longer detectable in large-budded YF cells. Surprisingly, GFP-Bud9p was also found to be highly concentrated at the tip of the growing bud throughout cell division. In contrast to GFP-Bud8p, no GFP-Bud9p was detectable at the mother-bud neck of small budded cells, and only a weak fluorescent signal was detectable at this region in large-budded cells. Moreover, GFP-Bud9p was already found to be highly concentrated at the distal pole of unbudded cells, indicating that Bud9p concentrates at the site of the incipient bud in G1.

Subcellular localization of epitope-tagged versions of Bud8p and Bud9p was analyzed by indirect immunofluorescence microscopy to corroborate the data found with GFP-fusion proteins. A triple myc epitope tag was inserted just after start codons of BUD8 and BUD9. The corresponding fusion genes were either expressed from high copy plasmids or were integrated into the genome of wild-type as well as 


\section{A}
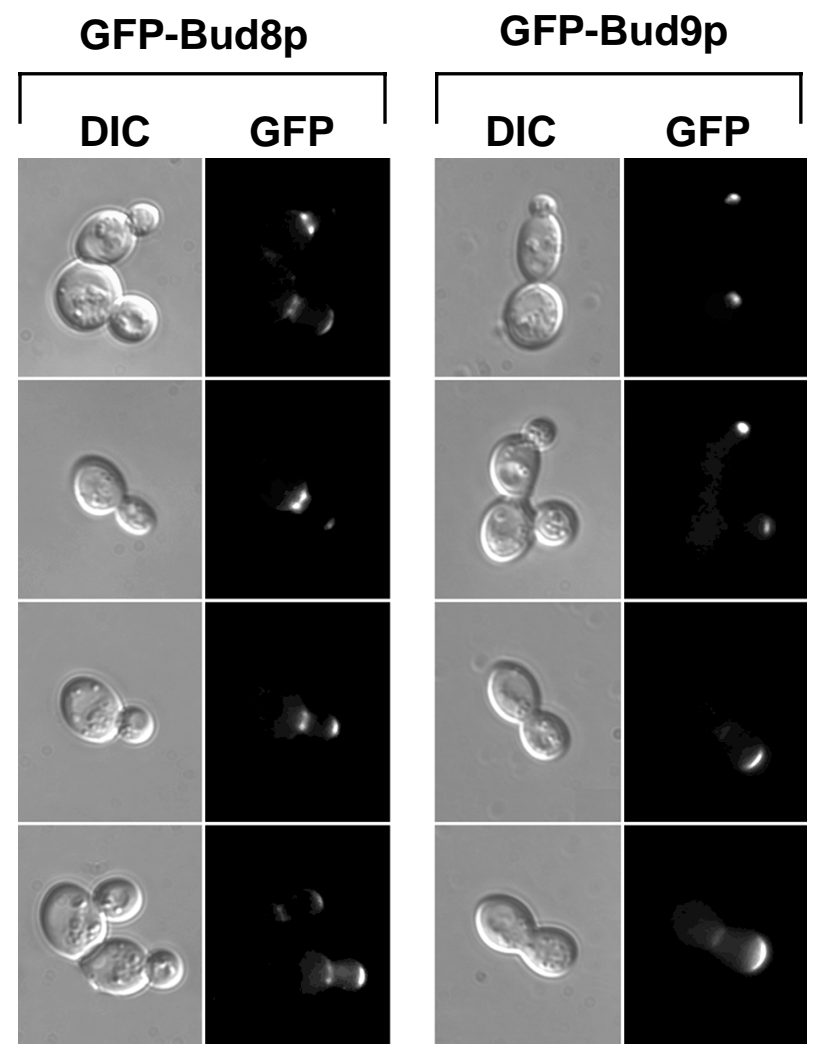

B
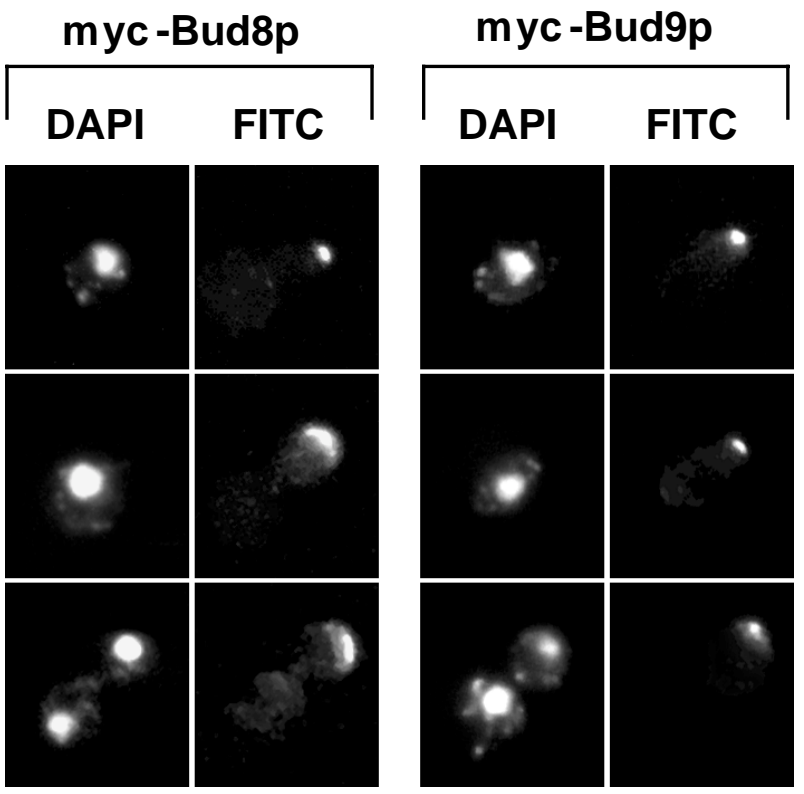


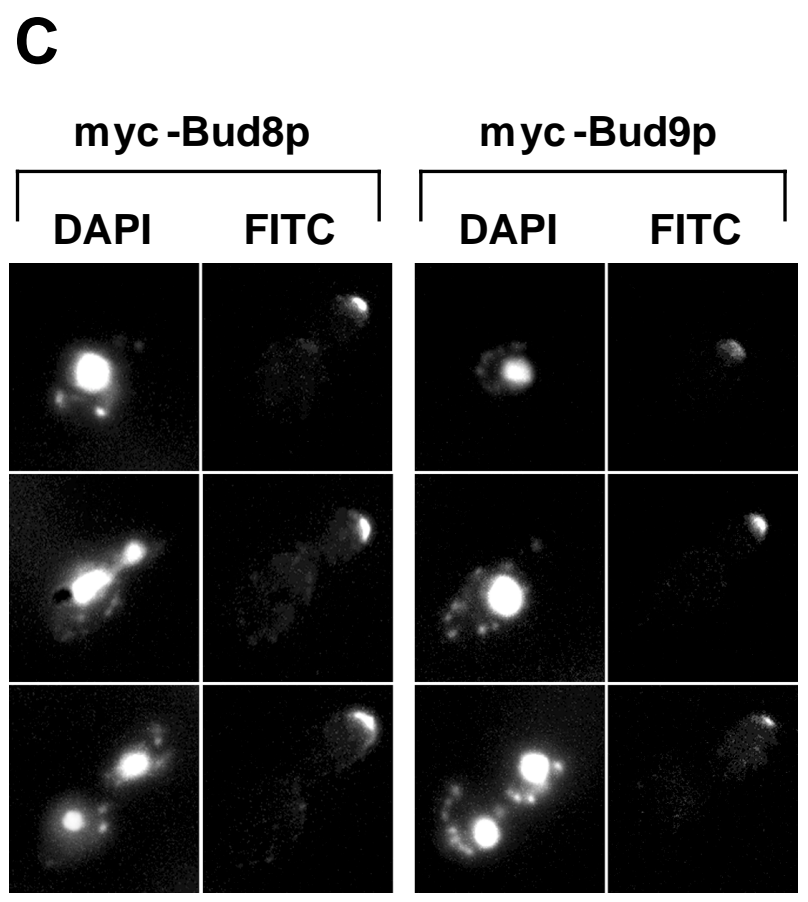

Figure 8. Subcellular localization of Bud8p and Bud9p in YF cells. (A) Shown are representative cells of wild-type strain RH2447 expressing either GFP-Bud8p from plasmid pME1772 or GFPBud9p from plasmid pME1777. Strains were grown in high ammonium media to exponential growth phase. Living cells at different stages of the cell cycle were chosen for photography according to their bud size and were viewed by both differential interference contrast microscopy (DIC) or by fluorescence microscopy (GFP). Identical results were obtained when expressing GFP-Bud8p or GFPBud9p under control of the MET25-promoter using plasmid pME1773 or pME1778, respectively. Scale bar applies to (A), (B) and (C) and equals $5 \mu \mathrm{m}$. (B) Immunofluorescence microscopy. Strain RH2447 expressing myc-Bud8p (pME1775) or myc-Bud9p (pME1780) was grown to exponential growth phase and prepared for anti-myc immunofluorescence. Shown are representative cells that were viewed for nuclear DNA with DAPI-imaging (DAPI) or for anti-myc immunofluorescence (FITC). (C) Anti-myc immunofluorescence microscopy of strains expressing myc-Bud8p (RH2491) or myc-Bud9p (RH2493) at endogenous levels. Shown are representative cells viewed for nuclear DNA with DAPI-imaging (DAPI) or for anti-myc immunofluorescence (FITC).

bud8 or bud9 mutant strains, to obtain endogenous expression levels of tagged proteins. Phenotypic analysis of tagged versions of BUD8 and BUD9 in bud8 and bud9 diploid mutant strains revealed no difference when compared to non-tagged versions. The localization pattern of myc-Bud8p was similar to that observed using GFP-Bud8p throughout the cell cycle (Figures $8 \mathrm{~B}$ and $8 \mathrm{C}$ ). In contrast to GFPBud8p, however, myc-Bud8p was found predominantly at the tip of growing cells and only very weak staining was visible at the mother-bud neck. Similar results were found for myc-Bud9p when compared to GFP-Bud9p. The epitope-tagged version of Bud9p was highly concentrated at the site of the incipient bud in unbudded cells and at the tip of the growing daughter cells. 
Expression of $B U D 8$ and $B U D 9$ is highly regulated during the cell cycle, with $B U D 8$ showing peak expression in M phase and BUD9 peaking in G1 (Spellman et al., 1998). Therefore, GFP-Bud8p and GFP-Bud9p subcellular localization was further analyzed when expressed from the MET25-promoter, to test whether cell cycle specific expression is important for localization of Bud8p or Bud9p. However, no differences were found when compared to GFP-BUD8 or GFP-BUD9 being under the control of the endogenous $B U D 8$ - or $B U D 9$-promoters.

Localization of Bud8p and Bud9p was further measured in haploid strains using GFP-fusions and myc-tagged versions. Interestingly, localization and expression patterns of Bud8p and Bud9p in haploids were found to be identical to that obtained in diploids, although haploid strains displayed an axial budding pattern (data not shown). This suggests that in haploid cells asymmetrically localized Bud8p and Bud9p proteins are not sufficient for induction of bipolar budding, most likely due to the presence of the haploid-specific budding proteins that might override functions of Bud8p and Bud9p.

Selection of the distal pole as site of cell division requires the presence of Bud8p and is favored by the absence of Bud9p

Previous studies have addressed the function of $B U D 8$ and $B U D 9$ by use of only point mutations or partial gene disruptions (Zahner et al., 1996; Mösch and Fink, 1997). Therefore, we constructed homozygous diploid strains carrying full deletions of $B U D 8$ or $B U D 9$, and analyzed their budding patterns in both YF and in $\mathrm{PH}$ cells by staining of bud scars (Figure 9). In addition, time-lapse microscopy was used, in order to distinguish between unipolar proximal (at the birth end of the cell) and unipolar distal (at the site opposite to the birth end) budding patterns (Figure 10). Full deletion of BUD8 caused a unipolar proximal budding pattern in both YF cells and PH filaments, whereas bud site selection of a control strain was bipolar in YF cells and switched to unipolar distal in PH filaments (Figure 9 and Figure 10). 
A
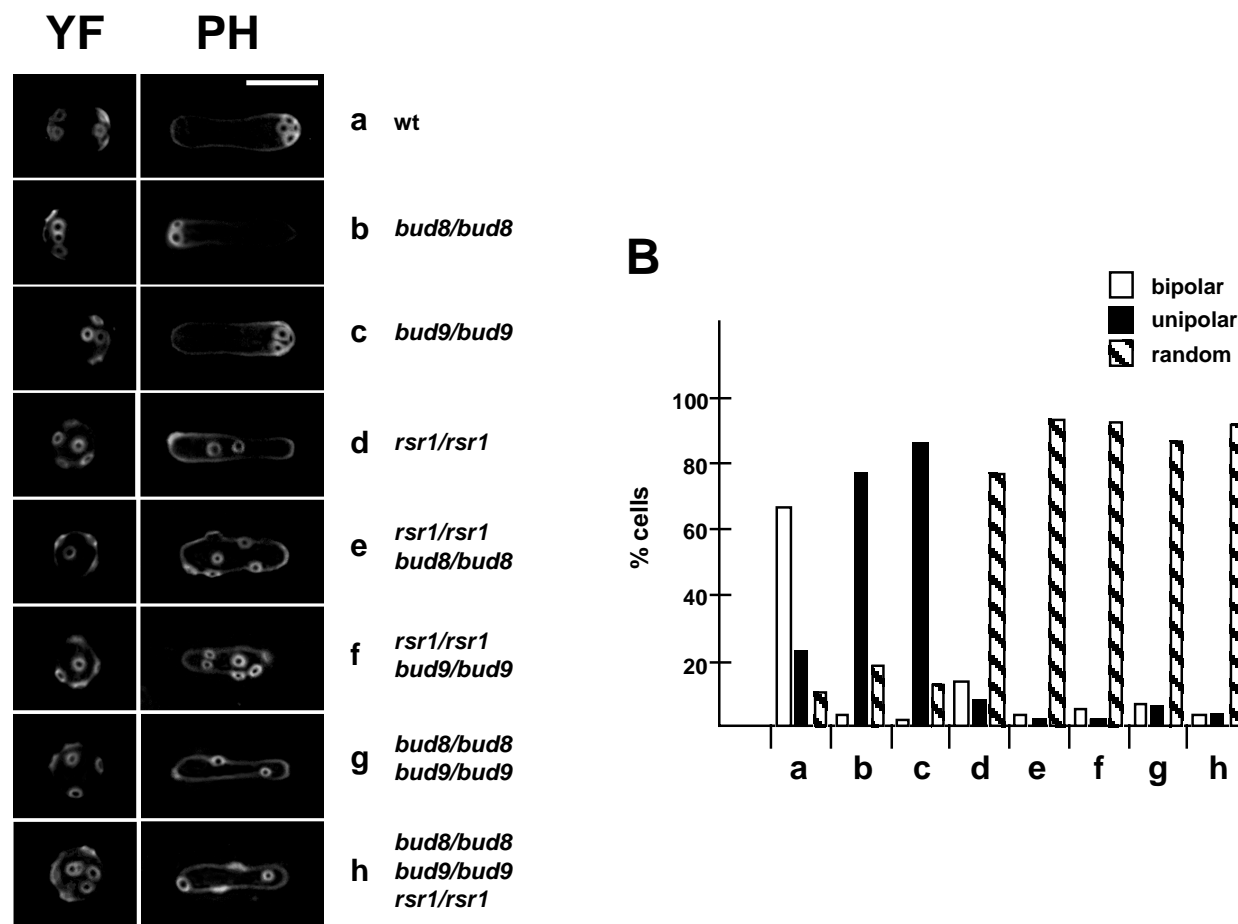

Figure 9. Regulation of bud site selection by $B U D 8, B U D 9$ and $R S R 1 / B U D 1$ in diploid YF and PH cells. (A) Fluorescence imaging of bud scar distribution of YF and PH cells after staining bud scars with calcofluor. Shown are representative cells of strains RH2447 (wt) (a), RH2449 (bud8/bud8) (b), RH2450 (bud9/bud9) (c), RH2448 (rsrl/rsrl) (d), RH2451 (rsrl/rsrl bud8/bud8) (e), RH2452 (rsrl/rsrl bud9/bud9) (f), RH2453 (bud8/bud8 bud9/bud9) (g), RH2454 (rsrl/rsrl bud8/bud8 bud9/bud9) (h). For YF cells, strains were transformed with plasmid pRS316 and grown to exponential growth phase in high ammonium media before staining with calcofluor. For PH cells , strains were transformed with plasmid pCG38 overexpressing PHD1 and grown in SLAD/LA media for $15 \mathrm{~h}$. Scale bar equals $5 \mu \mathrm{m}$. (B) Quantitative analysis of bud scar distribution. At least $200 \mathrm{YF}$ cells of strains described in (A) were analyzed for bud scar distribution (see Materials and Methods). Bars represent the percentage of cells exhibiting a bipolar (open bars), unipolar (solid bars) or random (hatched bars) budding pattern.

In agreement with earlier observations, full deletion of BUD 8 completely suppressed the formation of pseudohyphal filaments when tested on nitrogen starvation media (Figure 11). A detailed analysis of pseudohyphal sub-phenotypes revealed that the formation of long pseudohyphal cells and substrate invasive growth was similar in wild-type and in bud8 diploid mutants (Table I). Thus, changes of cell shape or switching from surface to invasive growth do not require BUD8. The buddingspecific function of Bud8p is supported by the fact that overexpression of BUD8 from high copy plasmids or from the MET25-promoter significantly enhanced the frequency of distal budding in YF cells without affecting cell morphology or invasive growth (data not shown). Deletion of $B U D 9$ led to preferentially unipolar distal budding in YF cells (Figure 9). In contrast to BUD8, however, we could not 
detect significant alterations in bud site selection patterns by overexpression of BUD9. In PH cells, the unipolar distal pattern was not influenced by the absence of BUD9 (Figure 9 and Figure 10).
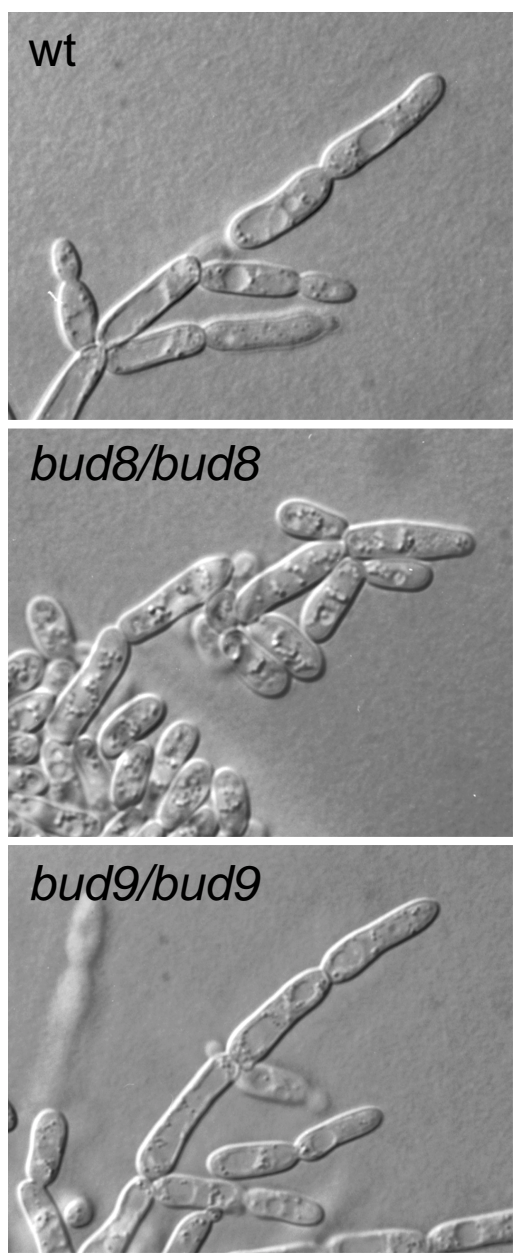

First buds

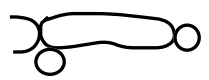

prox dist

$0 \% \quad 100 \%$

$73 \% \quad 27 \%$

Figure 10. Time-lapse observation of PH development. S. cerevisiae strains RH2447 (wt), RH2449 (bud8/bud8) and RH2450 (bud9/bud9) all carrying plasmid pCG38 were analyzed for selection patterns of first buds of virgin pseudohyphal cells. For each strain, at least 70 cell divisions were observed using a chamber for high magnification imaging of yeast growth on solid SLAD media (Kron et al., 1994). Numbers given indicate the percentage of virgin PH cells producing their first bud at either their birth end (proximal site) or opposite to their birth end (distal pole). After 3 days of growth, pseudohyphal development of cells at the edges of the colonies was visualized under the microscope using Nomarski optics. Scale bar equals $5 \mu \mathrm{m}$.

As a consequence, bud9/bud9 diploids produce regular amounts of pseudohyphae when grown on nitrogen starvation media (Figure 11). As found for Bud8p, Bud9p was not required for changes in cell morphology or invasive growth behavior during pseudohyphal development (Table I). This is in agreement with the fact that bud $9 /$ bud 9 mutant strains do not produce pseudohyphal filaments on nitrogen rich 
media, although the absence of $B U D 9$ induces unipolar distal budding already in the YF.

In summary, selection of the distal pole as site of cell division requires the presence of Bud8p and is favored by the absence of Bud9p. This suggests that Bud8p is as landmark for bud initiation at the distal pole, whereas Bud9p appears to inhibit distal budding.
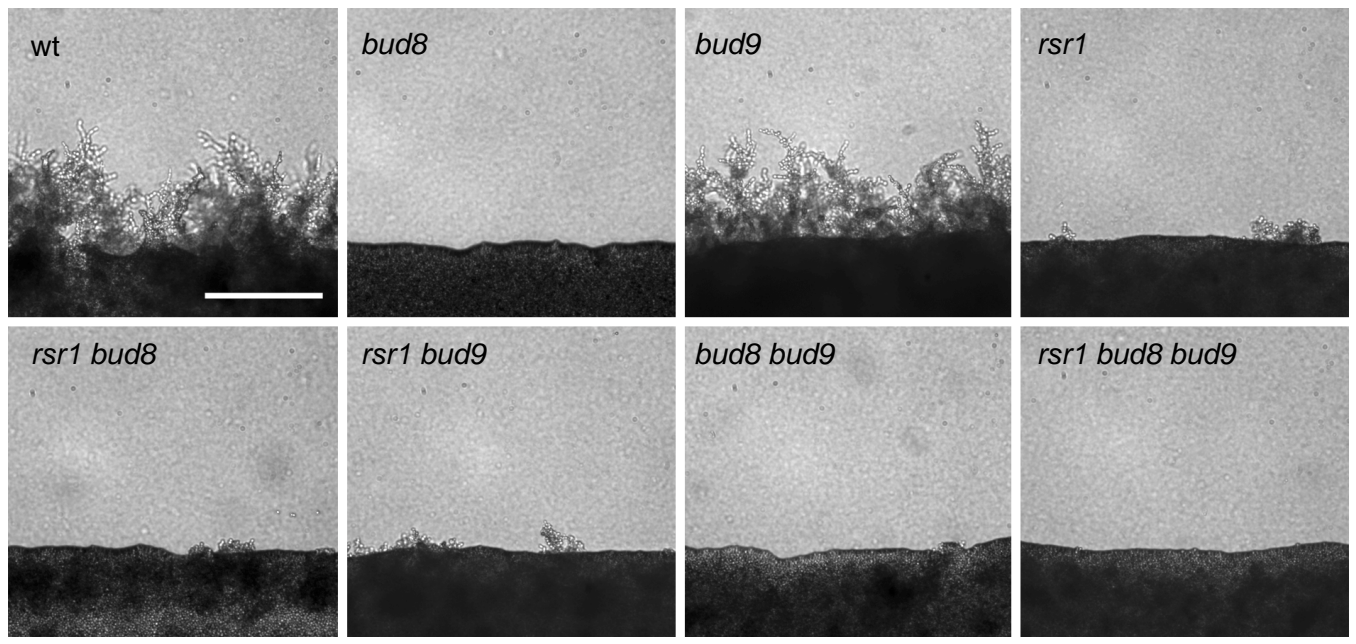

Figure 11. Regulation of pseudohyphal development by $R S R 1 / B U D 1, B U D 8$ and BUD9. Diploid strains homozygous for the indicated genotype and transformed with pRS316 were grown on nitrogen starvation media: wt (RH2447), bud8 (RH2449), bud9 (RH2450), rsrl (RH2448), rsrl bud8 (RH2451), rsrl bud9 (RH2452), bud8 bud9 (RH2453), rsrl bud8 bud9 (RH2454). After 4 days of growth, pseudohyphal development of strains was visualized under a microscope and photographed. The scale bar equals $100 \mu \mathrm{m}$.

Table I. Regulation of pseudohyphal development by RSR1/BUD1, BUD8 and BUD 9

\begin{tabular}{|c|c|c|c|c|c|c|c|}
\hline & & vant gen & & & & & \\
\hline Strain & $\begin{array}{l}\text { RSRl/ } \\
\text { BUDI }\end{array}$ & BUD8 & $B U D 9$ & Invasion & $\begin{array}{l}\% \text { long } \\
\mathrm{PH}\end{array}$ & $\begin{array}{l}\% \text { oval } \\
\text { YF }\end{array}$ & $\begin{array}{c}\% \text { round } \\
\mathrm{YF}\end{array}$ \\
\hline RH2447 & + & + & + & +++ & 25 & 61 & 14 \\
\hline RH2448 & - & + & + & +++ & 26 & 67 & 7 \\
\hline RH2449 & + & - & + & ++ & 22 & 65 & 13 \\
\hline RH2450 & + & + & - & +++ & 29 & 64 & 7 \\
\hline RH2451 & - & - & + & ++ & 25 & 63 & 12 \\
\hline RH2452 & - & + & - & +++ & 25 & 66 & 9 \\
\hline RH2453 & + & - & - & ++ & 21 & 60 & 19 \\
\hline RH2454 & - & - & - & ++ & 17 & 71 & 12 \\
\hline
\end{tabular}




\section{Bud8p and Bud9p proteins associate in vivo}

Because Bud8p and Bud9p proteins are co-localized at the distal bud and both affect distal bud site selection, we tested whether Bud8p and Bud9p proteins physically interact in vivo. For this purpose, in-frame fusions between GST and BUD8 or GST and $B U D 9$ were constructed and expressed from the GAL1-promoter together with myc epitope-tagged versions of either BUD8 or BUD9. Fusion proteins were induced and purified with glutathione-beads to isolate each fusion and any associated proteins. Proteins purified by glutathione-agarose were analyzed by western-blot analysis using polyclonal anti-GST antibodies or monoclonal anti-myc epitope antibodies. We found that myc-Bud8p co-purifies with GST-Bud9p, but not with GST alone (Figure 12). Vice versa, myc-Bud9p is associated with GST-Bud8p, but not with the GST control. Thus, Bud8p and Bud9p proteins are associated in vivo, suggesting that Bud8p and Bud9p might influence each other's function.

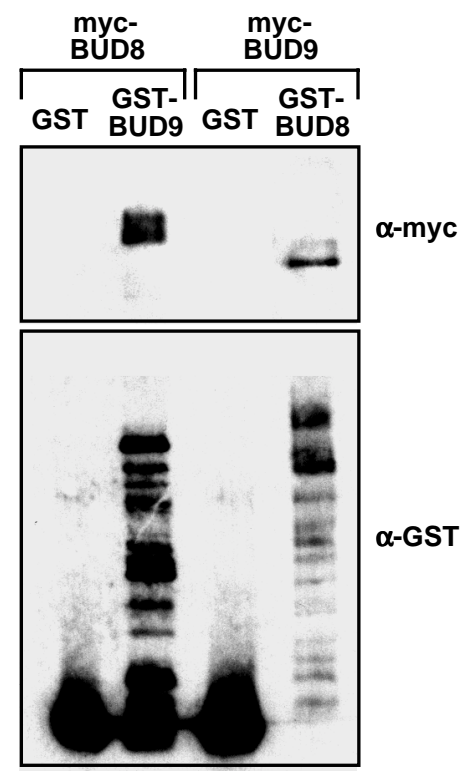

Figure 12. Co-purification of GST-Bud9p with myc-Bud8p and of GST-Bud8p with myc-Bud9p. Total protein extracts were prepared from strain RH2495 carrying either of the plasmid pairs pME1937 (myc-BUD8) and pYGEX-2T (GST), or pME1937 (myc-BUD8) and pME1941 (GSTBUD9), or pME1939 (myc-BUD9) and pYGEX-2T (GST), or pME1939 (myc-BUD9) and pME1940 (GST-BUD8). GST and GST-fusion proteins were purified as described. Equivalent amounts of each sample were subjected to SDS-PAGE, transferred to nitrocellulose, and probed with a monoclonal anti-myc antibody $(\alpha-m y c)$ or a polyclonal anti-GST antibody ( $\alpha-G S T)$. 


\section{Diploid bud8 $\Delta$ bud9 $\Delta$ null mutants produce a random budding pattern comparable to $\operatorname{rsr} 1 \Delta /$ bud $1 \Delta$ strains}

$B U D 8$ and $B U D 9$ are yet the only known genes encoding functions as diploidspecific landmarks at cell poles. It has been observed that diploid bud8 bud9 double mutants exhibit the unipolar proximal budding pattern of bud8 single mutants, suggesting the existence of further factors acting as bipolar landmarks (Zahner et al., 1996). However, the above study was performed with strains carrying point mutations in BUD8 and BUD9. Therefore, we here constructed homozygous diploid bud 8 bud 9 double mutant strains carrying full deletions of the BUD 8 and BUD9 open reading frames to reexamine these results. We found that diploid bud8 bud9 null mutants behave differentially to single mutants and are similar to $\mathrm{rsrl} / \mathrm{budl}$ strains, because they produce a random budding pattern in YF and PH cells (Figure 9). As controls, we also constructed homozygous diploid $\mathrm{rsr} / \mathrm{budl}$ single and $\mathrm{rsr} / \mathrm{budl}$ bud8 bud9 triple mutant strains. We found that bud8 bud9 double mutants are undistinguishable from the $\mathrm{rsrl} / \mathrm{budl}$ single or $\mathrm{rsrl} / \mathrm{budl}$ bud8 bud9 triple mutants with respect to bud site selection patterns or pseudohyphal development (Figure 9, Figure 11 and Table I). These results suggest that in diploid cells BUD 8 and BUD 9 encode the only gene products that act as bipolar landmarks at the cell poles of YF or PH cells.

\section{Mutations in RSR1/BUD1 are epistatic to mutations in BUD8 and BUD9 and Rsr1p/Bud1p is not required for unipolar localization of Bud8p or Bud9p}

Both Bud8p and Bud9p have been suggested to function as landmarks at the cell poles of diploid yeast cells that might recruit or locally activate the Rsr1p/Bud1p GTP-binding protein (Chant, 1999). To test this hypothesis, we constructed homozygous diploid bud8 and bud9 mutant strains in combination with mutations in RSR1/BUD1. We predicted that if RSR1/BUD1 acts downstream of BUD8 and BUD9 in the bud site selection pathway, mutations in RSR1/BUDI should be epistatic over mutations in either BUD8 or BUD9. As described above, rsrl/budl bud8 and rsrl/budl bud9 double mutant strains were assayed for bud site selection patterns in YF and PH cells (Figure 9). In addition, pseudohyphal filament formation along with a detailed analysis of $\mathrm{PH}$ cell morphogenesis and substrate invasive growth was investigated (Figure 11 and Table I). We found that both the $r s r l / b u d l$ bud8 and rsrl/budl bud9 double mutants were indistinguishable from the $r s r l / b u d l$ single mutant with respect to all phenotypes measured. This result argues for RSR1/BUDI 
acting downstream of $B U D 8$ or $B U D 9$ in the control of bud site selection of both YF and PH cells.

Our genetic analysis indicated that $R S R 1 / B U D 1$ acts downstream of $B U D 8$ and $B U D 9$. This assumption could be verified, if $\operatorname{Rsr} 1 \mathrm{p} / \mathrm{Bud} 1 \mathrm{p}$ was not required for asymmetric localization of Bud8p and Bud9p. Therefore, subcellular localization of GFP-Bud8p and GFP-Bud9p was analyzed in a homozygous diploid $r s r 1 / b u d 1$ mutant strain (Figure 13A). Indeed, no obvious difference of subcellular localization of GFP-Bud8p or GFP-Bud9p was observed in a $r s r 1 / b u d l$ mutant when compared to a control strain, further confirming Rsr1p/Bud1p to act downstream of Bud8p and Bud9p. Similarly, we tested localization of GFP-Bud8p in diploid bud9 mutants and GFP-Bud9p in diploid bud8 strains (Figure 13B). Again, no differences could be detected when compared to localization of the GFP-fusion proteins in a control strain. These findings are in agreement with the genetic studies predicting that in the absence of Bud9p, Bud8p should be normally localized to the distal bud site thereby allowing the unipolar distal pattern found in the bud9 single mutant. Vice versa, the absence of Bud8p was not expected to affect localization of Bud9p, because absence of both Bud8p and Bud9p (in a bud8 bud9 double mutant strain) was found to cause random and not unipolar proximal budding as exhibited by the bud8 single mutant.

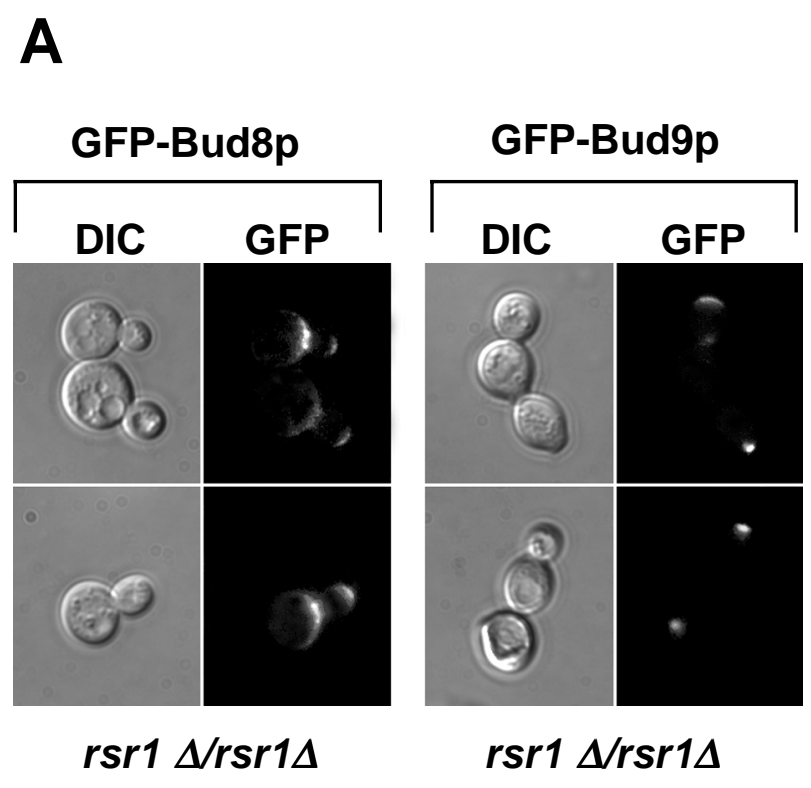


B

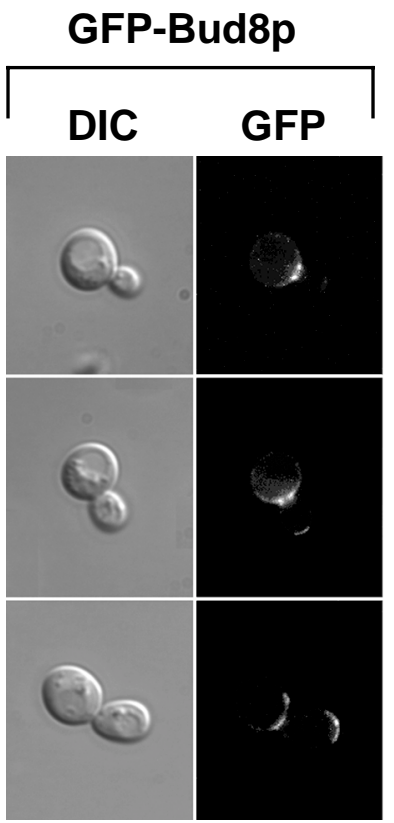

bud94/bud94

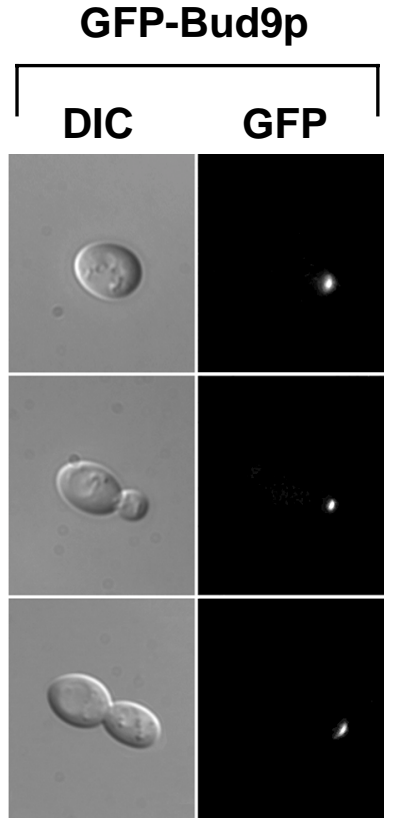

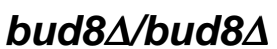

Figure 13. Unipolar localization of GFP-Bud8p and GFP-Bud9p is independent of RSR1/BUD1, BUD8 or BUD9. (A) Living cells of strain RH2448 (rsr1 $/ r s r 1 \Delta$ ) expressing GFP-Bud8p (pME1772) or GFP-Bud9p (pME1777) grown in high ammonium media to exponential growth phase were viewed by both differential interference contrast microscopy (DIC) or by fluorescence microscopy (GFP). Identical results were obtained when expressing GFP-Bud8p or GFP-Bud9p under control of the MET25-promoter using plasmid pME1773 or

pME1778, respectively. (B) Subcellular localization of GFP-Bud8p and GFP-Bud9p in strains

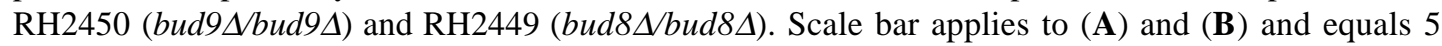
$\mu \mathrm{m}$.

Starvation for nitrogen initiates unipolar distal cell divisions in pseudohyphal filaments by preventing localization of Bud9p, but not Bud8p, at the distal cell pole

During switching from the YF to the PH filamentous form, the budding pattern of diploid strains switches from bipolar to unipolar distal. Therefore, we wanted to know whether Bud8p or Bud9p are directly involved in this process. Our genetic data and localization studies suggested that Bud8p is a landmark at the distal cell pole that is required for distal bud site selection. In contrast, Bud9p appears to act as an inhibitor of distal bud site selection, because absence of Bud9p (in a bud9 mutant) favors unipolar distal budding in YF cells. For this reason, PH cells can be viewed as YF cells lacking Bud9p with respect to their budding pattern. We reasoned that $\mathrm{PH}$ cells might differ from YF cells by their expression patterns of the BUD9 gene or by subcellular localization of the Bud9 protein. Therefore, subcellular localization of 
GFP-Bud8p and GFP-Bud9p were measured under nitrogen starvation conditions that favor pseudohyphal development. In addition, expression levels of BUD8 and $B U D 9$ genes as well as intracellular levels of GFP-Bud8p and GFP-Bud9p proteins were determined under these conditions. Nitrogen starvation did not significantly alter subcellular localization of GFP-Bud8p (Figure 14) when compared to nonstarved cells (Figure 8).
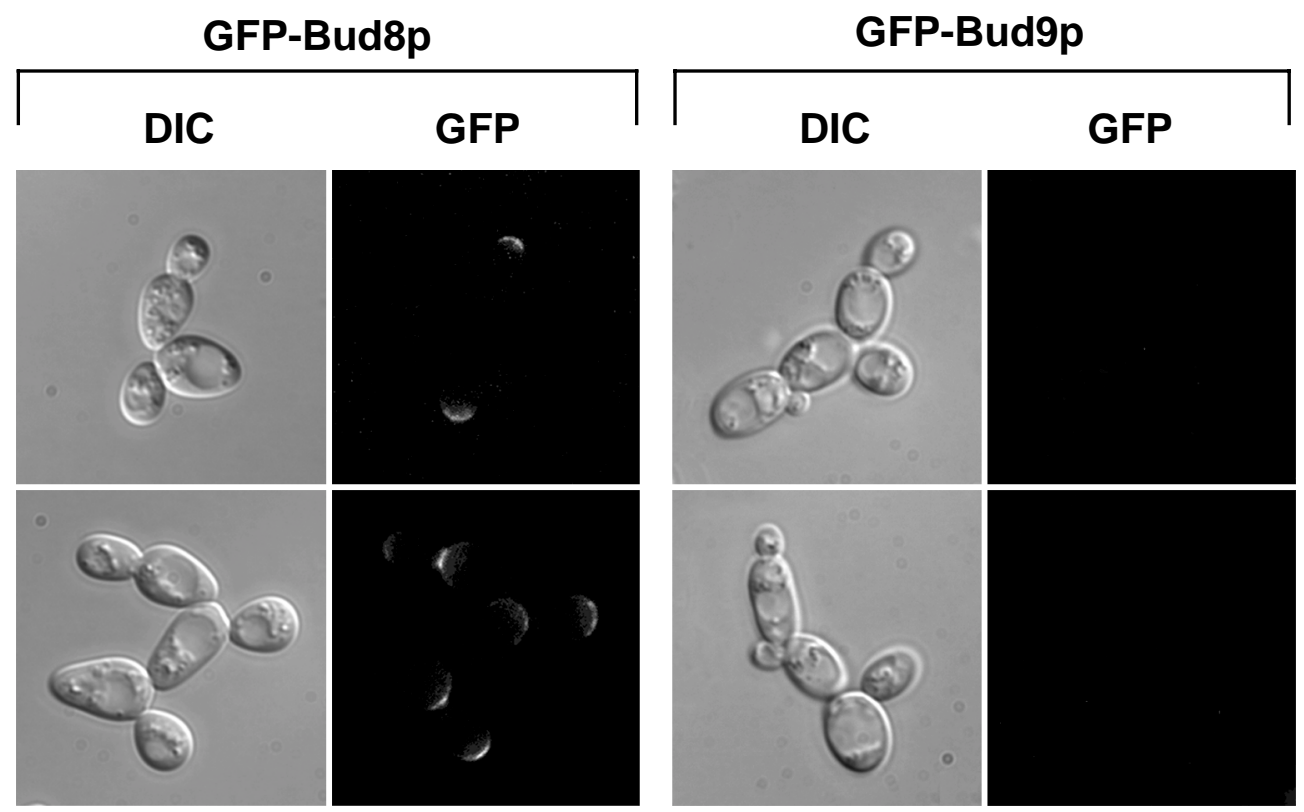

Figure. 14. Subcellular localization of GFP-Bud8p and GFP-Bud9p in living PH cells. Shown are representative cells of wild-type strain RH2447 expressing either GFP-Bud8p from plasmid pME1772 or GFP-Bud9p from plasmid pME1777. S. cerevisiae strains were grown in low ammonium media (SLAD/LA) for $15 \mathrm{~h}$. Living cells were viewed by both differential interference contrast microscopy (DIC) or by fluorescence microscopy (GFP). Scale bar equals $5 \mu \mathrm{m}$.

GFP-Bud8p was still found to be concentrated to the tip of the growing bud as well as to the mother-bud neck region. Expression of GFP-Bud8p in nitrogen-starved cultures did hardly change, but a two-fold induction of steady-state BUD 8 mRNA was measured under these conditions (Figure 15). However, localization of GFPBud9p to the distal cell pole was completely suppressed when cultures were starved for nitrogen (Figure 14). In contrast, intracellular protein levels of GFP-Bud9p or $B U D 9$ mRNA levels in these cultures did not drop more than by a factor of roughly two (Figure 15). 
A
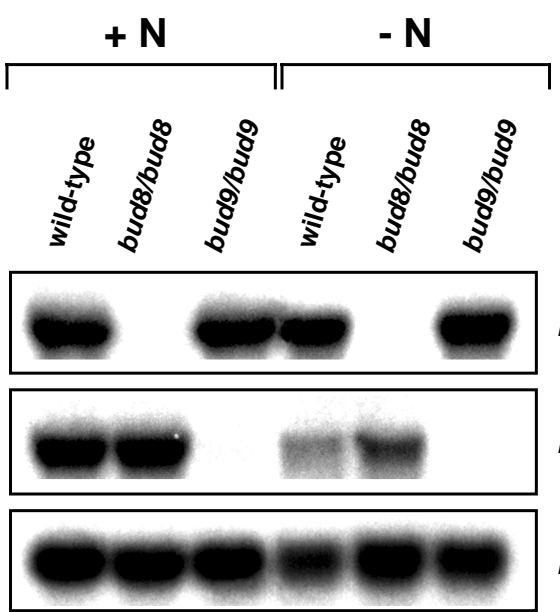

BUD8

BUD9

PDA1

$\begin{array}{ccccccc}100 & - & 104 & 182 & - & 194 & \frac{B U D 8}{P D A 1} \\ 100 & 105 & - & 46 & 54 & - & \frac{B U D 9}{P D A 1}\end{array}$

B

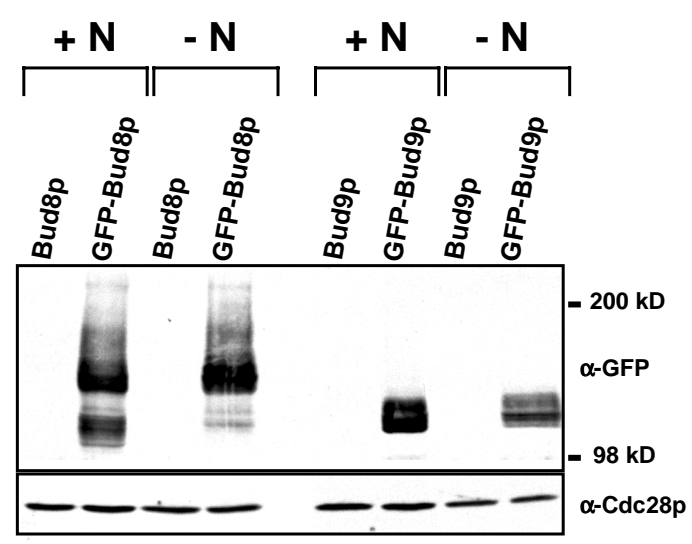

Fig. 15. Regulation of $B U D 8$ and $B U D 9$ expression by nitrogen availability. (A) Autoradiogram showing steady state levels of $B U D 8$ and $B U D 9$ mRNA of strains RH2447 (wild-type), RH2449 (bud8/bud8) and RH2450 (bud9/bud9) all carrying plasmid pRS316 for Ura ${ }^{+}$prototrophy and either grown to exponential growth phase in high ammonium media $(+\mathrm{N})$ or grown in low ammonium media (- N) for $15 \mathrm{~h}$. Expression of the $P D A 1$ gene served as an internal control. Relative expression levels of BUD8 (BUD8/PDA1) and BUD9 (BUD9/PDA1) are shown below and were obtained using a Phosphor-Imaging scanner. Numbers represent mean values of three independent measurements and were obtained by normalizing $B U D$ gene transcript levels with respect to $P D A 1$ and to $B U D$ gene expression in wild-type strain $\mathrm{RH} 2447$ grown on high ammonium $(+\mathrm{N})$. Standard deviation was below 20\%. (B) Regulation of GFP-Bud8p and GFP-Bud9p fusion protein levels by nitrogen. Total protein extracts were prepared from strain RH2447 carrying either of the plasmid pME1783 (Bud8p), pME1772 (GFP-Bud8p), pME1784 (Bud9p) or pME1777 (GFP-Bud9p) grown in high ammonium (+ $\mathrm{N}$ ) or low ammonium (- N) media. Extracts were analyzed for expression of GFP-fusion proteins by Western blot analysis using a polyclonal anti-GFP antibody ( $\alpha$-GFP). As an internal control, protein levels of Cdc28p were measured in the same extracts using a polyclonal anti-Cdc28p antibody $(\alpha-$ Cdc28p).

Thus, although significant levels of GFP-Bud9p were still present in nitrogen-starved cells, none of this protein was found to be concentrated at the distal bud tip during cell division. Similar results were obtained with the epitope-tagged myc-Bud8p and myc-Bud9p proteins (data not shown). These findings suggest that the starvationinduced switch of cell polarity from bipolar budding of YF cells to unipolar distal budding of $\mathrm{PH}$ cells is achieved by a mechanism that prevents Bud9p from being localized to the distal cell pole. 


\section{Discussion}

Upon starvation for nitrogen, diploid yeast cells switch polarity from the bipolar to the unipolar distal pattern that is a prerequisite for the formation of linear filaments during pseudohyphal growth (Gimeno et al., 1992; Kron et al., 1994). Our study provides novel evidence for a molecular model that explains how Bud8p and Bud9p regulate polarity of diploid yeast cells in response to nutrients (Figure 16).

\section{distal cell pole}

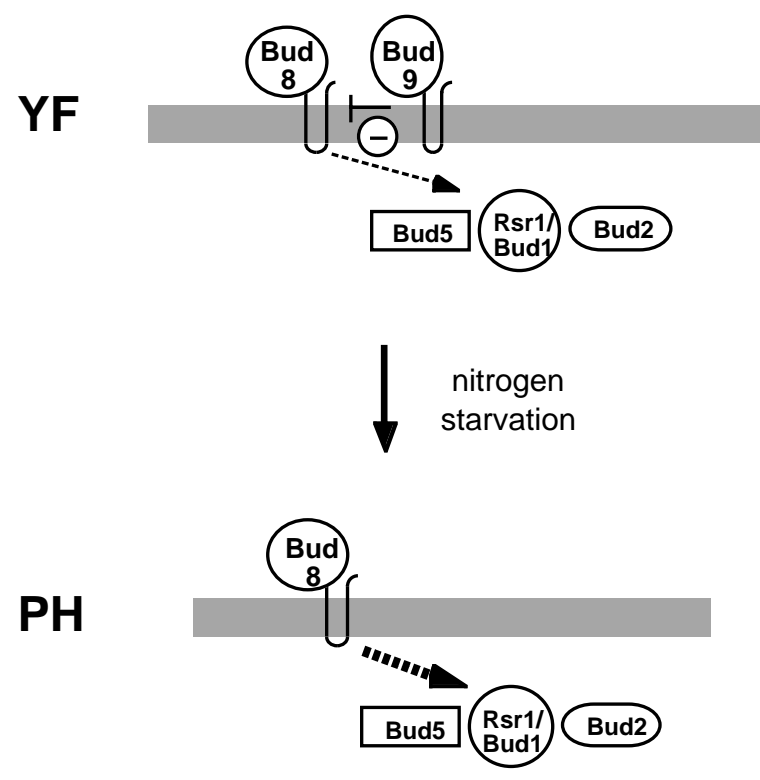

Figure. 16. Model for regulation of bud site selection at the distal cell pole of S. cerevisiae. In YF cells, Bud9p is localized at the distal cell pole and interferes with Bud8p-mediated bud site selection via the Rsr1p/Bud1p-Bud5p-Bud2p GTPase module. In PH cells, nutritional starvation for nitrogen prevents distal localization of Bud9p allowing efficient Bud8p-mediated distal budding.

We propose that Bud8p is a cortical tag at the distal pole of both YF and PH cells, where it directs bud initiation. When nutrients are available, Bud9p is also localized to the distal pole, where it significantly reduces the potential of distal bud site selection. As consequence, YF cells elaborate the bipolar budding pattern. In response to nitrogen starvation, Bud9p (but not Bud8p) is mislocalized and therefore no more present at the distal cell pole. This leads to unipolar distal cell divisions in $\mathrm{PH}$ cells, a budding pattern that can be mimicked in YF cells by deletion of BUD9. Because Bud8p and Bud9p associate in vivo, Bud9p might be an inhibitor of Bud8pmediated distal bud site selection. Both Bud8p and Bud9p are predicted to be transmembrane proteins. As previously discussed (Chant, 1999), Bud8p and Bud9p 
consist of N-terminal extracellular domains (515 and 460 amino acids, respectively), membrane spanning domains, short cytoplasmic loops (42 and 38 amino acids), second membrane spanning domains, and short ( 3 and 2 amino acids) extracellular domains at the C-terminus (Figure 16). Membrane association of Bud8p and Bud9p is in agreement with the fact that membrane dissolving detergents are required for full extraction of both proteins (see Materials and methods). In addition, deletion of the predicted transmembrane domains of Bud8p inhibits both its function and proper intracellular localization (our unpublished results). Surprisingly, neither Bud8p nor Bud9p have a predicted signal sequence, although their N-terminal domains contain a number of predicted N-glycosylation sites. Both Bud8p and Bud9p might be glycosylated, because their apparent molecular weights are much higher than calculated when analyzed by SDS PAGE (Figure 15). However, whether Bud8p and Bud9p are glycoproteins that are delivered to the cell surface via the secretory pathway, similar to Ax12p/Bud10p (Halme et al., 1996; Roemer et al., 1996; Sanders et al., 1999), remains to be elucidated.

Our study defines the distal pole as main site of action for Bud8p and Bud9p in YF and PH cells. However, highly concentrated amounts of GFP-Bud8p and some GFPBud9p were also detectable at the bud neck in living cells. Because the bud neck is positioned between the distal cell pole of the mother and the proximal pole of the daughter, careful analysis of bud neck staining of a given protein is necessary for interpretation of its function. Our localization studies clearly show that GFP-Bud8p and GFP-Bud9p proteins residing at the bud neck are asymmetrically concentrated at the mother-side of the bud neck (Figure 8).

Moreover, we could never observe dividing cells with GFP-Bud8p concentrated at the distal pole of the growing bud and at the proximal pole of the mother. Thus, we prefer the view that this portion of Bud8p and Bud9p is localized at the distal cell pole of the mother, and not at the proximal pole of the daughter. This argues for Bud8p and Bud9p functioning not only at the distal pole of the new daughter, but as well at the distal pole of the mother.

How does Bud8p act as a cortical tag? Our study provides genetic evidence that the general budding factor Rsr1p/Bud1p might be recruited to or locally be activated at the distal pole through Bud8p, because rsrl/budl mutants display a random budding pattern independently of Bud8p. Vice versa, Bud8p is localized to the distal bud site irrespective of the presence or absence of Rsr1p/Bud1p. However, a direct interaction between Bud8p and Rsr1p/Bud1p seems unlikely, because Rsr1p/Bud1p is distributed uniformly around the plasma membrane (Michelitch and Chant, 1996). 
Thus, Bud8p might control distal bud site selection via regulatory proteins of Rsr1p/Bud1p, e.g. Bud2p or Bud5p (Park et al., 1999).

An important finding of our study is that neither protein levels nor subcellular localization of Bud8p undergo significant changes when cells are starved for nitrogen and switch to the PH form. Yet, BUD8 is absolutely required for pseudohyphal development. Thus, Bud8p does not appear to be a prime regulator that controls the switch from bipolar to unipolar cell divisions in response to nitrogen starvation. Rather, the efficiency of Bud8p as a cortical tag might be altered in PH cells, by e.g. postranslational modification or by interaction with an inhibitor. We favor Bud9p to be a negative regulator of distal budding, whose subcellular localization is under control of nitrogen starvation. This view of Bud9p is supported by several observations. (i) Bud9p is highly concentrated at the distal pole of YF cells, whereas absence of Bud9p (in bud9 deletion strains) favors distal bud initiation. This finding per se defines a negative function for Bud9p at the distal pole, given the assumption that the main localization of Bud9p reflects its major site of action. (ii) Bud8p and Bud9p co-purify, indicating physical interaction in vivo. (iii) $\mathrm{PH}$ cells display an unipolar distal pattern much like YF bud9 mutant cells, thus naturally reflecting the artificial situation created by deletion of $B U D 9$. (iv) In $\mathrm{PH}$ cells, Bud9p is prevented from being localized to its presumed site of negative action, the distal cell pole. How does Bud9p fulfill such a negative function? As discussed above, the Bud9p sequence predicts an extracellular domain of 460 amino acids at the $\mathrm{N}$-terminus and a short loop of 38 amino acids at the inside of the cell. Thus, Bud9p might interact directly with Bud8p and prevent Bud8p from recruiting downstream factors to the distal pole. Alternatively, Bud9p might compete with Bud8p for these downstream proteins, but negatively act on their function. It remains to be determined whether such a mechanism involves posttranslational modifications of Bud9p or alterations of the machinery that recognizes and asymmetrically localizes Bud9p to the distal pole.

\section{Materials and methods}

\section{Yeast strains and growth conditions}

All yeast strains used in this study are congenic to the $\Sigma 1278$ b genetic background

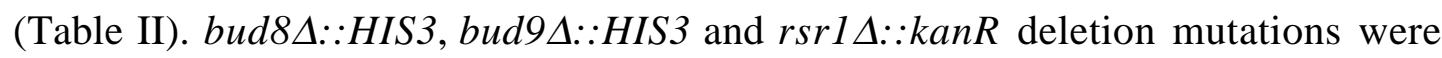
introduced using plasmids pME1767, pME1768 and pME1766 (Table III). Strains RH2491, RH2492, RH2493 and RH2494, all expressing myc-epitope-tagged versions of either $B U D 8$ or $B U D 9$ at endogenous levels, were obtained by integration 
of linearized plasmids pME1936 or pME1938 into the ura3-52 locus. Standard methods for genetic crosses and transformation were used and standard yeast culture YPD, YNB and SC media were prepared essentially as described (Guthrie and Fink, 1991). Low ammonium medium (SLAD) was prepared as described (Gimeno et al., 1992). Solid SLAD 2\% agar medium was used for qualitative and quantitative pseudohyphal growth assays. Strains were grown in liquid SLAD layered over SLAD 2\% agar in Petri plates (SLAD/LA) essentially as described (Kron et al., 1994) for bud scar staining and GFP-fluorescence microscopy of PH cells as well as for isolation of RNA and protein extracts from PH cells. The PHDI PH-inducer was overexpressed from plasmid pCG38 in strains used for bud scar staining and timelapse microscopy to obtain a high proportion of $\mathrm{PH}$ cells required for these measurements.

\section{Plasmids}

Plasmids pME1766, pME1767 and pME1768 carrying the rsr1 $4:: k a n R$, bud $8 \Delta:: H I S 3$ and bud9A::HIS3 deletion cassettes were created by replacement of the coding sequences of $R S R 1 / B U D 1, B U D 8$ and $B U D 9$ for either the HIS3 selectable marker or the kanR kanamycin resistance gene using a PCR-based 3-step cloning strategy. To obtain genomic fragments carrying BUD8 and BUD9, plasmids pRS202BUD8 and pRS202-BUD9 were isolated from a yeast genomic library in pRS202 (from P. Hieter, University Of British Columbia, Vancouver, Canada) using colony hybridizations and ${ }^{32} \mathrm{P}$-radiolabeled probes for BUD8 and BUD9.

Plasmid pME1769 was obtained by subcloning of a $4.0 \mathrm{~kb}$ BamHI-XhoI genomic fragment from pRS202-BUD8 into pRS316 (Sikorski and Hieter, 1989), and plasmid pME1783 by subcloning of a $3.1 \mathrm{~kb}$ BamHI-ScaI fragment from pRS202-BUD8 into pRS426 (Christianson et al., 1992). Plasmid pME1771 and pME1772, both expressing GFP-Bud8p from the BUD8-promoter, were constructed by introducing a $B g l$ II site in front of the second codon of BUD8 and insertion of a 750-bp BglIIfragment encoding the GFPuv variant of GFP that was amplified from plasmid pBAD-GFPuv (CLONTECH, Heidelberg, Germany).

Plasmid pME1773 expressing GFP-Bud8p from the MET25-promoter was obtained by subcloning of a $3.3 \mathrm{~kb}$ EcoRV-ScaI fragment carrying GFP-BUD 8 from pME1771 into p426MET25 (Mumberg et al., 1994). Plasmids pME1775, pME1936 and pME1937, all expressing a triple myc eptitope-tagged version of Bud8p under the control of the BUD8-promoter, were obtained by insertion of a $120 \mathrm{bp} \mathrm{BamHI}$ fragment carrying the triple myc epitope $\left(\mathrm{myc}^{3}\right)$ after the start codon of $B U D 8$. Plasmid pME1770 and pME1784 were obtained by subcloning of a $5.6 \mathrm{~kb}$ EcoRI 
genomic fragment from pRS202-BUD9 into either pRS316 or pRS426. Plasmids pME1776 and pME1777 both expressing GFP-Bud9p from the BUD9-promoter were constructed by introducing a BamHI site in front of the second codon of $B U D 9$ and insertion of the GFPuv cassette described above. pME1778 expressing GFP-Bud9p from the MET25-promoter was obtained by subcloning of a $3 \mathrm{~kb} B a m \mathrm{HI}-E c o \mathrm{RI}$ BUD9 fragment into p426MET25 and insertion of the GFPuv BglII cassette. Plasmids pME1780, pME1938 and 1939, expressing a triple myc eptitope-tagged version of Bud9p under the control of the $B U D$ 9-promoter, were obtained by insertion of the triple myc epitope after the start codon of $B U D$ 9. Plasmids pME1940 and pME1941 were obtained by N-terminal fusion of BUD8 and BUD9 open reading frames to GST in vector pYGEX-2T (Schlenstedt et al., 1995).

\section{Qualitative and quantitative assays of pseudohyphal growth}

Qualitative assays for pseudohyphal development were performed as described previously (Mösch et al., 1996). After 3 days of growth on solid SLAD medium, pseudohyphal colonies were viewed with a Zeiss Axiovert microscope and photographed using a Xillix Microimager digital camera and the Improvision Openlab software (Improvision, Coventry, England). Quantitative assays for PH growth including determination of substrate invasion and cell shape were performed following the protocols described earlier (Mösch and Fink, 1997).

\section{Bud scar staining and determination of budding patterns}

Bud scar staining was performed on YF and PH cells grown to exponential growth phase. YF cells in exponential growth phase were prepared by growing strains in liquid $\mathrm{YNB}$ medium at $30^{\circ} \mathrm{C}$ to $\mathrm{OD}_{600}$ of 0.6 . $\mathrm{PH}$ cells in exponential growth phase were obtained by growth on SLAD/LA medium. Routinely, $5 \times 10^{5}$ cells were inoculated into $10 \mathrm{ml}$ SLAD liquid medium layered over $10 \mathrm{ml}$ SLAD $2 \%$ agar in Petri dishes and incubated at $30^{\circ} \mathrm{C}$. After $15 \mathrm{~h}$, cells were suspended and collected by centrifugation in conical polystyrene tubes. YF and PH cell suspensions were fixed at room temperature for $2 \mathrm{~h}$ in $3.7 \%$ formaldehyde. Samples were rinsed twice in water and resuspended in $200 \mu \mathrm{l}$ of a fresh stock of $1 \mathrm{mg} / \mathrm{ml}$ calcofluor white (Fluorescent Brightener F-6259, Sigma). Bud scars were visualized by fluorescence microscopy using a Zeiss Axiovert microscope and photographed using a Xillix Microimager digital camera and the Improvision Openlab software (Improvision, Coventry, England). Cells with two to 10 obvious bud scars were divided into three classes: bipolar, cells with two or more bud scars with at least one scar at each end of the cell (the birth end and the free end); unipolar, cells with all bud scars at one end of the 
cell immediately adjacent to one another; random, cells with bud scar distributions other than bipolar or unipolar. Numbers in the tables represent the percentage of cells in each class for a sample of at least 200 cells.

\section{Time-lapse microscopy}

Bud site selection of growing PH filaments was determined by using a chamber for high magnification imaging of yeast growth as described previously (Kron et al., 1994). Positions of bud site emergence were determined by direct microscopic observation. For each strain measured, at least 70 cell divisions were observed.

\section{GFP-fluorescence and indirect immunofluorescence microscopy}

Yeast strains harboring plasmids encoding GFP-Bud8p or GFP-Bud9p were grown to exponential growth phase in high or low ammonium media as described for bud scar staining. Cells from $1 \mathrm{ml}$ of the cultures were harvested by centrifugation and immediately viewed in vivo on a Zeiss axiovert microscope by either differential interference contrast microscopy or fluorescence microscopy using a GFP filter set (AHF Analysentechnik AG, Tübingen, Germany). Cells were photographed using a Xillix Microimager digital camera and the Improvision Openlab software (Improvision, Coventry, England). For immunofluorescence microscopy, cells were cultured as for GFP-microscopy, fixed in 3.7\% formaldehyde, and spheroblasts were prepared as described (Pringle et al., 1991). DAPI (4,6-diamino-2-phenylindole) staining and monoclonal mouse anti-myc antibodies (9E10) together with an Alexa 488-conjugated goat-anti-mouse antibody (Molecular Probes Inc., Eugene, OR, USA) were used for visualization of nuclei and myc epitope-tagged proteins, respectively. Cells were viewed and photographed as described above using standard DAPI and FITC filter sets.

\section{Northern blot analysis}

Total RNA was prepared from cultures grown in high or low ammonium media exactly as described for bud scar staining and according to the method described previously (Cross and Tinkelenberg, 1991). Total RNA was separated on a $1.4 \%$ agarose gel containing 3\% formaldehyde and transferred onto nylon membranes as described earlier (Mösch et al., 1992). BUD8, BUD9 and PDAl transcripts were detected using gene specific ${ }^{32} \mathrm{P}$-radiolabeled DNA probes. Hybridizing signals were quantified using a BAS-1500 Phosphor-Imaging scanner (Fuji). 


\section{Protein analysis}

Whole cell extracts: Extracts were prepared from cultures grown to exponential growth phase in high or low ammonium medium as described above. Briefly, cultures were washed in ice-cold buffer $\mathrm{R}$ (50 mM Tris-Cl [pH 7.5], $1 \mathrm{mM}$ EDTA, $50 \mathrm{mM}$ DTT), lysed with glass beads in $200 \mu \mathrm{l}$ buffer R + PIM (1 mM of each PMSF, TLCK, TPCK, p-aminobenzamidine- $\mathrm{HCl}$ and o-phenantroline) $+3 \%$ Triton $\mathrm{X}-100+0.8 \% \mathrm{SDS}$ at $4^{\circ} \mathrm{C}$, and spun at $3500 \mathrm{rpm}$ for $5 \mathrm{~min}$ to remove glass beads and large cell debris. $10 \mu \mathrm{l}$ of the extracts were removed to determine total protein concentration using a protein assay kit from (BIO-RAD, München, Germany). SDS loading dye was added to the remaining total extracts and proteins were denatured by heating at $37^{\circ} \mathrm{C}$ for $5 \mathrm{~min}$. Equal amounts of proteins were then subjected to SDSPAGE electrophoresis and transferred to nitrocellulose membranes. GFP-fusion proteins and Cdc28p were detected using ECL technology (Amersham, Buckinghamshire, United Kingdom) after incubation of membranes with either a rabbit polyclonal anti-GFP antibody (CLONTECH, Heidelberg, Germany) or rabbit polyclonal anti-Cdc28p antibodies (a kind gift of S. Irniger, Georg-AugustUniversity Göttingen, Germany) and a peroxidase-coupled goat anti-rabbit IgG secondary antibody (Dianova, Hamburg, Germany). Purification of GST fusions: Extracts of strains expressing GST-fusion proteins together with myc-tagged versions of Bud8p or Bud9p were prepared after growth on galactose medium for 6 hours exactly as previously described (Roberts et al., 1997). Extracts were incubated with glutathione agarose overnight at $4^{\circ} \mathrm{C}$, and beads were repeatedly washed and collected to purify GST fusions and any associated proteins. Samples were denatured by heating at $60^{\circ} \mathrm{C}$ for $5 \mathrm{~min}$ in SDS loading dye, and equal amounts of each sample were analyzed by Western-blot analysis as described above using either polyclonal anti-GST antibodies (Santa Cruz Biotechnologies, Santa Cruz, CA, USA) or the monoclonal mouse anti-myc antibody (9E10). 
Table II. Strains used in this study

\begin{tabular}{|c|c|c|}
\hline Strain & Genotype & Source \\
\hline RH2447 & $\begin{array}{l}\text { MATa/MAT } \alpha, \text { ura3-52/ura3-52, } \\
\text { leu2::hisG/LEU2, trp1::hisG/TRP1 }\end{array}$ & This study \\
\hline RH2448 & $\begin{array}{l}\text { MATa/MAT } \alpha, \text { rsr } 1 \Delta:: k a n R / r s r 1 \Delta:: k a n R, \\
\text { ura3-52/ura3-52, leu2::hisG/LEU2, trp1::hisG/TRP1 }\end{array}$ & This study \\
\hline RH2449 & 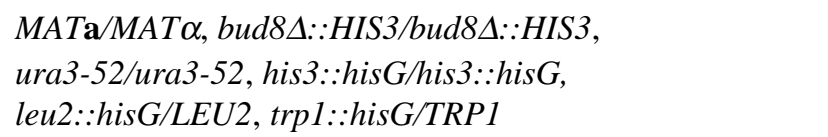 & This study \\
\hline RH2450 & 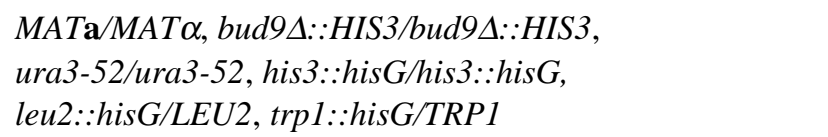 & This study \\
\hline RH2451 & 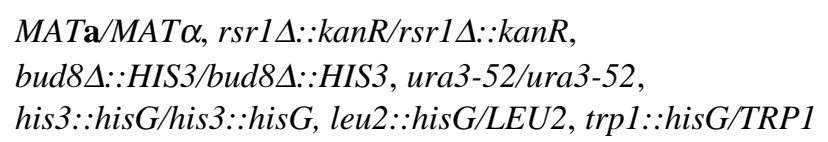 & This study \\
\hline RH2452 & 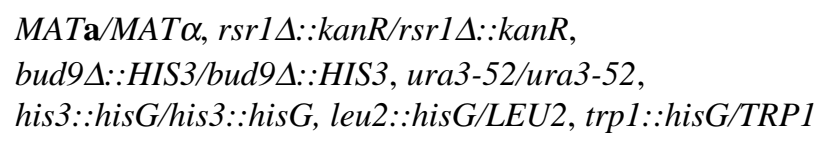 & This study \\
\hline RH2453 & 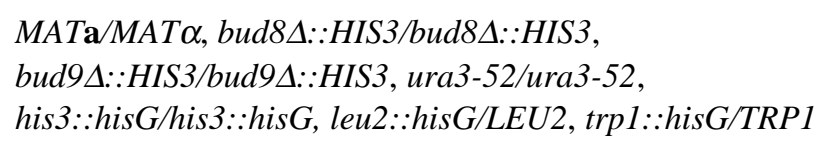 & This study \\
\hline RH2454 & 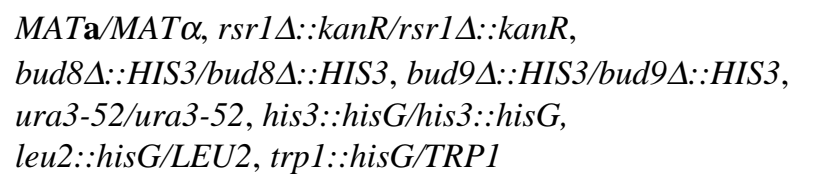 & This study \\
\hline RH2491 & $\begin{array}{l}\text { MATa/MAT } \alpha, \text { myc-BUD8-URA3/ } \\
\text { myc-BUD8-URA3, ura3-52/ura3-52, } \\
\text { leu2::hisG/LEU2, trp1::hisG/TRP1 }\end{array}$ & This study \\
\hline RH2492 & 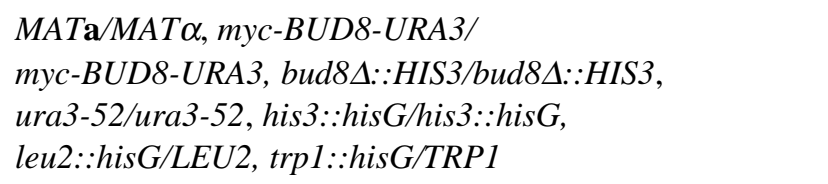 & This study \\
\hline RH2493 & $\begin{array}{l}\text { MATa/MAT } \alpha, \text { myc-BUD9-URA3/ } \\
\text { myc-BUD9-URA3, ura3-52/ura3-52, } \\
\text { leu2::hisG/LEU2, trp1::hisG/TRP1 }\end{array}$ & This study \\
\hline RH2494 & $\begin{array}{l}\text { MATa/MAT } \alpha, \text { myc-BUD9-URA3/ } \\
\text { myc-BUD9-URA3, bud9 } \Delta:: H I S 3 / \text { bud9 } 9:: H I S 3, \\
\text { ura3-52/ura3-52, his3::hisG/his3::hisG, } \\
\text { leu2::hisG/LEU2, trp1::hisG/TRP1 }\end{array}$ & This study \\
\hline RH2495 & $\begin{array}{l}\text { MATa/MATa, ura3-52/ura3-52, } \\
\text { leu2::hisG/leu2::hisG, his3::hisG/HIS3, } \\
\text { trp1::hisG/TRP1 }\end{array}$ & This study \\
\hline
\end{tabular}


Table III. Plasmids used in this study

\begin{tabular}{|c|c|c|}
\hline Plasmid & Description & Reference \\
\hline pME1766 & $r s r 1 \Delta:: k a n R$ cassette for full deletion of $R S R 1$ & This study \\
\hline pME1767 & bud $8 \Delta:: H I S 3$ cassette for full deletion of $B U D 8$ & This study \\
\hline pME1768 & bud9 $9:: H I S 3$ cassette for full deletion of $B U D 9$ & This study \\
\hline pME1769 & 4.0-kb fragment containing BUD 8 in pRS316 & This study \\
\hline pME1770 & 5.6-kb fragment containing $B U D 9$ in pRS316 & This study \\
\hline YCp(RSR1) & 1.6-kb fragment containing $R S R 1$ in $\mathrm{YCp} 50$ & (Ruggieri et al., 1992) \\
\hline pME1771 & BUD8prom-GFP-BUD8 fusion in pRS316 & This study \\
\hline pME1772 & BUD8prom-GFP-BUD8 fusion in pRS426 & This study \\
\hline pME1773 & MET25prom-GFP-BUD8 fusion in pRS426MET25 & This study \\
\hline pME1775 & BUD8prom-myc ${ }^{3}-B U D 8$ fusion in pRS426 & This study \\
\hline pME1776 & BUD9prom-GFP-BUD9 fusion in $\mathrm{pRS} 316$ & This study \\
\hline pME1777 & BUD9prom-GFP-BUD9 fusion in pRS426 & This study \\
\hline pME1778 & MET25prom-GFP-BUD9 fusion in pRS426MET25 & This study \\
\hline pME1780 & BUD9prom-myc ${ }^{3}-B U D 9$ fusion in pRS426 & This study \\
\hline pME1783 & 3.1-kb fragment containing $B U D 8$ in pRS426 & This study \\
\hline pME1784 & 5.6-kb fragment containing $B U D 9$ in pRS426 & This study \\
\hline pME1936 & BUD8prom-myc ${ }^{3}-B U D 8$ fusion in pRS306 & This study \\
\hline pME1937 & BUD8prom-myc ${ }^{3}-B U D 8$ fusion in pRS425 & This study \\
\hline pME1938 & BUD9prom-myc ${ }^{3}-B U D 9$ fusion in pRS306 & This study \\
\hline pME1939 & BUD9prom-myc ${ }^{3}-B U D 9$ fusion in pRS425 & This study \\
\hline pME1940 & GAL1prom-GST-BUD8 fusion in pYGEX-2T & This study \\
\hline pME1941 & GAL1prom-GST-BUD9 fusion in pYGEX-2T & This study \\
\hline pRS316 & URA3-marked centromere vector & (Sikorski and Hieter, 1989 \\
\hline pRS426 & $U R A 3$-marked $2 \mu \mathrm{m}$ vector & (Christianson et al., 1992) \\
\hline p426MET25 & $\begin{array}{l}\text { pRS } 426 \text { containing MET25-promoter and } \\
\text { CYC1 terminator }\end{array}$ & (Mumber g et al., 1994) \\
\hline pCG38 & 2.6-kb fragment containing $P H D 1$ in pRS202 & (Gimeno and Fink, 1994) \\
\hline pYGEX-2T & $\begin{array}{l}\text { URA3-marked } 2 \mu \mathrm{m} \text { GALIprom-GST fusion } \\
\text { vector }\end{array}$ & (Schlenstedt et al., 1995) \\
\hline
\end{tabular}




\section{References}

Bender, A. and Pringle, J.R. (1989) Multicopy suppression of the $c d c 24$ budding defect in yeast by $C D C 42$ and three newly identified genes including the rasrelated gene RSR1. Proc. Natl. Acad. Sci. USA, 86, 9976-9980.

Chant, J. (1999) Cell polarity in yeast. Annu. Rev. Cell Dev. Biol., 15, 365-391.

Chant, J., Corrado, K., Pringle, J.R. and Herskowitz, I. (1991) Yeast BUD5, encoding a putative GDP-GTP exchange factor, is necessary for bud site selection and interacts with bud formation gene BEM1. Cell, 65, 1213-1224.

Chant, J. and Herskowitz, I. (1991) Genetic control of bud site selection in yeast by a set of gene products that constitute a morphogenetic pathway. Cell, 65, 12031212.

Chant, J. and Pringle, J.R. (1995) Patterns of bud-site selection in the yeast Saccharomyces cerevisiae. J. Cell Biol., 129, 751-765.

Christianson, T.W., Sikorski, R.S., Dante, M., Shero, J.H. and Hieter, P. (1992) Multifunctional yeast high-copy-number shuttle vectors. Gene, 110, 119-122.

Crameri, A., Whitehorn, E.A., Tate, E. and Stemmer, W.P. (1996) Improved green fluorescent protein by molecular evolution using DNA shuffling. Nat. Biotechnol., 14, 315-319.

Cross, F.R. and Tinkelenberg, A.H. (1991) A potential positive feedback loop controlling $C L N 1$ and $C L N 2$ gene expression at the start of the yeast cell cycle. Cell, 65, 875-883.

Freifelder, D. (1960) Bud position in Saccharomyces cerevisiae. J. Bacteriol., 80, 567-568.

Fujita, A., Oka, C., Arikawa, Y., Katagai, T., Tonouchi, A., Kuhara, S. and Misumi, Y. (1994) A yeast gene necessary for bud-site selection encodes a protein similar to insulin-degrading enzymes. Nature, 372, 567-570.

Gimeno, C.J. and Fink, G.R. (1994) Induction of pseudohyphal growth by overexpression of PHD1, a Saccharomyces cerevisiae gene related to transcriptional regulators of fungal development. Mol. Cell. Biol., 14, 2100-2112.

Gimeno, C.J., Ljungdahl, P.O., Styles, C.A. and Fink, G.R. (1992) Unipolar cell divisions in the yeast $S$. cerevisiae lead to filamentous growth: regulation by starvation and RAS. Cell, 68, 1077-1090.

Guthrie, C. and Fink, G.R. (1991) Guide to Yeast Genetics and Molecular Biology. Methods Enzymol., 194.

Halme, A., Michelitch, M., Mitchell, E.L. and Chant, J. (1996) Bud10p directs axial cell polarization in budding yeast and resembles a transmembrane receptor. Curr. Biol., 6, 570-579. 
Hicks, J.B., Strathern, J.N. and Herskowitz, I. (1977) Interconversion of yeast mating types III. Action of the homothallism $(\mathrm{HO})$ gene in cells homozygous for the mating type locus. Genetics, 85, 395-405.

Kron, S.J., Styles, C.A. and Fink, G.R. (1994) Symmetric cell division in pseudohyphae of the yeast Saccharomyces cerevisiae. Mol. Biol. Cell, 5, 10031022.

Madden, K. and Snyder, M. (1992) Specification of sites for polarized growth in Saccharomyces cerevisiae and the influence of external factors on site selection. Mol. Biol. Cell, 3, 1025-1035.

Madden, K. and Snyder, M. (1998) Cell polarity and morphogenesis in budding yeast. Annu. Rev. Microbiol., 52, 687-744.

Michelitch, M. and Chant, J. (1996) A mechanism of Bud1p GTPase action suggested by mutational analysis and immunolocalization. Curr. Biol., 6, 446454.

Mösch, H.-U. and Fink, G.R. (1997) Dissection of filamentous growth by transposon mutagenesis in Saccharomyces cerevisiae. Genetics, 145, 671-684.

Mösch, H.-U., Graf, R. and Braus, G.H. (1992) Sequence-specific initiator elements focus initiation of transcription to distinct sites in the yeast TRP4 promoter. EMBO J., 11, 4583-4590.

Mösch, H.-U., Roberts, R.L. and Fink, G.R. (1996) Ras2 signals via the Cdc42/Ste20/mitogen-activated protein kinase module to induce filamentous growth in Saccharomyces cerevisiae. Proc. Natl. Acad. Sci. USA, 93, 5352-5356.

Mumberg, D., Müller, R. and Funk, M. (1994) Regulatable promoters of Saccharomyces cerevisiae: comparison of transcriptional activity and their use for heterologous expression. Nucleic Acids Res., 22, 5767-5768.

Park, H.O., Bi, E., Pringle, J.R. and Herskowitz, I. (1997) Two active states of the Ras-related Bud1/Rsr1 protein bind to different effectors to determine yeast cell polarity. Proc. Natl. Acad. Sci. USA, 94, 4463-4468.

Park, H.O., Chant, J. and Herskowitz, I. (1993) BUD2 encodes a GTPase-activating protein for Bud1/Rsr1 necessary for proper bud-site selection in yeast. Nature, 365, 269-274.

Park, H.O., Sanson, A. and Herskowitz, I. (1999) Localization of Bud2p, a GTPaseactivating protein necessary for programming cell polarity in yeast to the presumptive bud site. Genes Dev., 13, 1912-1917.

Pringle, J.R., Adams, A.E., Drubin, D.G. and Haarer, B.K. (1991) Immunofluorescence methods for yeast. Methods Enzymol., 194, 565-602. 
Roberts, R.L., Mösch, H.-U. and Fink, G.R. (1997) 14-3-3 proteins are essential for RAS/MAPK cascade signaling during pseudohyphal development in S. cerevisiae. Cell, 89, 1055-1065.

Roemer, T., Madden, K., Chang, J. and Snyder, M. (1996) Selection of axial growth sites in yeast requires Axl2p, a novel plasma membrane glycoprotein. Genes Dev., 10, 777-793.

Ruggieri, R., Bender, A., Matsui, Y., Powers, S., Takai, Y., Pringle, J.R. and Matsumoto, K. (1992) RSR 1, a ras-like gene homologous to Krev-1 (smg21A/rap1A): role in the development of cell polarity and interactions with the Ras pathway in Saccharomyces cerevisiae. Mol. Cell. Biol., 12, 758-766.

Sanders, S.L., Gentzsch, M., Tanner, W. and Herskowitz, I. (1999) O-Glycosylation of Ax12/Bud10p by Pmt $4 p$ is required for its stability, localization, and function in daughter cells. J. Cell Biol., 145, 1177-1188.

Schlenstedt, G., Saavedra, C., Loeb, J.D., Cole, C.N. and Silver, P.A. (1995) The GTP-bound form of the yeast Ran/TC4 homologue blocks nuclear protein import and appearance of poly(A)+ RNA in the cytoplasm. Proc. Natl. Acad. Sci. U.S.A., 92, 225-229.

Segall, J.E. (1993) Polarization of yeast cells in spatial gradients of alpha mating factor. Proc. Natl. Acad. Sci. USA, 90, 8332-8336.

Sikorski, R.S. and Hieter, P. (1989) A system of shuttle vectors and yeast host strains designed for efficient manipulation of DNA in Saccharomyces cerevisiae. Genetics, 122, 19-27.

Snyder, M. (1989) The SPA2 protein of yeast localizes to sites of cell growth. J. Cell Biol., 108, 1419-1429.

Spellman, P.T., Sherlock, G., Zhang, M.Q., Iyer, V.R., Anders, K., Eisen, M.B., Brown, P.O., Botstein, D. and Futcher, B. (1998) Comprehensive identification of cell cycle-regulated genes of the yeast Saccharomyces cerevisiae by microarray hybridization. Mol. Biol. Cell, 9, 3273-3297.

Valtz, N. and Herskowitz, I. (1996) Pea2 protein of yeast is localized to sites of polarized growth and is required for efficient mating and bipolar budding. J. Cell Biol., 135, 725-739.

Zahner, J.E., Harkins, H.A. and Pringle, J.R. (1996) Genetic analysis of the bipolar pattern of bud site selection in the yeast Saccharomyces cerevisiae. Mol. Cell. Biol., 16, 1857-1870. 


\title{
Chapter 3
}

\section{Type I myosin is required for selection of cell division sites in budding yeast and physically associates with the landmark protein Bud8p}

\begin{abstract}
Spatial selection of cell division sites in budding yeast Saccharomyces cerevisiae depends on actin and associated proteins that regulate polarization of the actin cytoskeleton and the deposition of spatial landmarks at the cell poles. Type I myosins are motor proteins that are associated with cortical actin patches in yeast, where they are thought to regulate polarized cell growth. We have investigated the role of Myo3p and Myo5p representing the yeast type I myosins in regulation of the bipolar bud site selection program. Deletion of either the MYO3 or MYO5 gene is sufficient to cause random budding in diploid strains. myo 3 or myo5 mutations are epistatic to mutations in $B U D 8$ or $B U D 9$, genes encoding bipolar landmark proteins that are localized at the cell poles. Localization of GFP-Bud8p and GFP-Bud9p is not affected by deletion of $M Y O 3$ or $M Y O 5$, placing myosin I function downstream of the bipolar landmarks. Furthermore, type I myosin Myo3p physically associates with Bud8p in vivo, but not with Bud9p. Our data suggest that type I myosins might control bud site selection by interaction with bipolar landmark proteins to induce site-specific actin assembly and cell growth.
\end{abstract}




\section{Introduction}

Establishment of cell polarity is essential for proliferation and development of most organisms. The budding yeast $S$. cerevisiae establishes cell polarity at several stages during cell division and is a useful model to study the molecular mechanisms that control cell polarization (Chant, 1996; Palmieri and Haarer, 1998; Pringle et al., 1995; Roemer et al., 1996). Yeast cells divide by budding and choose cell division sites in distinct spatial pattern that depend on their cell type. Haploid MATa and $M A T \alpha$ cells bud in an axial pattern, where mother cells bud adjacent to the previous bud site, and where daughter cells bud adjacent to the birth site. Diploid MATa/MAT $\alpha$ cells bud in a bipolar pattern, where buds form either at the pole opposite to their birth scar, called the distal pole, or they bud near their birth site, at the proximal pole.

Selection of yeast cell division sites is thought to be a process that includes at least three distinct steps (Bähler and Peter, 2000). First, cells choose a site on their surface, a spatial landmark, toward which they will polarize. In diploid cells, the transmembrane Bud8p and Bud9p proteins have been proposed to act as spatial landmarks for bipolar bud site selection (Chant, 1999; Harkins et al., 2001; Taheri et al., 2000). Bud8p is localized at the distal pole and is required for distal budding, suggesting that Bud8p tags the distal pole for initiation of cell division (Harkins et al., 2001; Taheri et al., 2000). Bud9p has been localized to both the proximal and the distal pole, but is required for proximal budding only. Thus, Bud9p may inhibit cell division at the distal pole and serve as a tag for proximal cell division (Taheri et al., 2000). In a second step, landmark proteins are thought to be recognized by a number of proteins that collectively are called polarity establishment proteins or actinorganizing complex (Chant, 1999; Kang et al., 2001; Marston et al., 2001; Park et al., 1999; Pringle et al., 1995; Roemer et al., 1996). Although this complex includes a growing list of proteins, no direct link has been established between one of its members and the bipolar landmark proteins Bud8p or Bud9p. In a third step, the actin-organizing complex is thought to recruit the machinery that organizes and polarizes the actin cytoskeleton to the site of the landmark (Bähler and Peter, 2000; Chant, 1999; Ni and Snyder, 2001; Sheu et al., 2000). As a consequence, cell wall and other materials are transported along the polarized actin cytoskeleton towards the landmark, leading to initiation of cell division and bud emergence.

A number of studies have shown that bud site selection directs polarization of the actin cytoskeleton to specific sites on the cell surface, but per se is not required for actin polarization. For instance, yeast mutants that lack $B U D 8$ and $B U D 9$ are 
suppressed for bipolar budding, but not for polarization of the actin cytoskeleton or cellular morphogenesis. In contrast, polarized cell growth, which describes the delivery of cell wall and other materials along the polarized actin cytoskeleton towards the apex of the growing bud, is critical not only for cell morphogenesis but also for the budding pattern. Actin and a number of actin-associated proteins are required for both apical cell growth and bipolar budding, demonstrating that proteins that modulate the structure of the actin cytoskeleton and polarized cell growth are also key regulators of bud site selection. For example, the formin protein Bnilp promotes the nucleation and assembly of actin filaments, which function as precursors for tropomyosin-stabilized cables that direct polarized cell growth (Sheu et al., 2000; Snyder, 1989; Zahner et al., 1996). It has been shown that depletion of Bnilp causes disassembly of cables and mislocalization of Bud8p (Harkins et al., 2001; Ni and Snyder, 2001). This suggests that the duration of actin polarization and apical growth are critical factors for establishing spatial landmarks, which are required for selection of budding sites in the next round of cell division.

Type I myosins are highly conserved single-headed motor proteins that function in numerous actin-based processes involved in cell growth and development (Evangelista et al., 2000; Goodson et al., 1996; Lechler et al., 2000; Pruyne and Bretscher, 2000). Budding yeast possesses two functionally redundant type I myosins that are encoded by the MYO3 and MYO5 genes. In haploid yeast, single deletion of either $M Y O 3$ or $M Y O 5$ does not result in any observable alterations in growth phenotype. Double deletions of MYO3 and MYO5 cause severe defects in polarized cell growth and endocytosis (Geli and Riezman, 1996; Goodson et al., 1996). Myo3p and Myo5p localize to cortical patches that are enriched at sites of polarized growth and overlap with actin patches (Evangelista et al., 2000; Goodson et al., 1996; Lechler et al., 2000; Pruyne and Bretscher, 2000). This suggests that type I myosins promote actin polymerization at sites of cell growth. This regulation of actin polymerization by Myo3p and Myo5p was shown to be achieved by interaction with the Arp2/3 complex that nucleates polymerization of actin filaments (Evangelista et al., 2000; Lechler et al., 2000). In vivo activity of Myo3p and Myo5p further depends on phosphorylation by Ste20p and Cla4p, two members of the family of PAK-like (p21-activated kinase) protein kinases (Wu et al., 1997). The fact that type I myosins affect polarized growth implies a possible involvement of these actin-regulating proteins in bud site selection. It has not been tested so far, whether Myo3p or Myo5p are required for bipolar budding of diploid yeast or for delivery of bipolar landmark proteins to the cell surface, as has been found in the case of Bnilp. 
Here, we investigated the genetic and biochemical interactions between type I myosins and the bipolar landmark proteins Bud8p and Bud9p in diploid yeast. We demonstrate, that deletion of either MYO3 or MYO5 alone is sufficient to induce a random budding pattern in diploid strains. myo3 myo5 double mutant strains are not viable. Genetic interaction studies reveals that myo3 and myo5 single mutations are epistatic to $b u d 8$ and $b u d 9$ mutations, placing myosin I function downstream of bipolar landmarks. This conclusion is supported by the fact that polar localization of GFP-Bud8p and GFP-Bud9p is not affected by deletions in MYO3 or MYO5. In addition, Myo3p physically associates with Bud8p in vivo. Our data suggest that bipolar landmark proteins might control bud site selection by interaction with type I myosins to direct actin assembly and polarized growth to the poles of yeast cells.

\section{Results}

\section{MYO3 and MYO5 are required for bipolar budding of diploid strains}

Previous studies have shown that double deletions of both the MYO3 and MYO5 genes in haploid strains cause severe growth defects and suppress the haploidspecific axial bud site selection program (Geli and Riezman, 1996; Goodson et al., 1996). Here, we determined whether type I myosins Myo3p and Myo5p are required for growth and bipolar bud site selection in diploid strains. For this purpose, we constructed homozygous diploid strains carrying full deletions of MYO3 or MYO5. Resulting strains did not exhibit any detectable growth defect. In contrast, strains carrying myo3 $\Delta y o 5 \Delta$ double deletions were not viable. Budding patterns of myo3 $\Delta$ myo3 $\Delta$ and myo5 $\Delta$ myo5 $\Delta$ single mutants were measured by staining of bud scars, to determine the effect of Myo3p or Myo5p depletion on bud site selection. We found that myo3 $\Delta$ myo3 $\Delta$ and myo5 $\Delta$ myo5 $\Delta$ deletion mutant strains had lost the typical bipolar budding pattern observed in a control strain and produced significantly higher amounts of cells with a random budding pattern (Figure 17). In case of myo3 $\Delta$ myo $3 \Delta$ strains, the percentage of random budding cells increased at least 8 -fold and in the myo5 $\Delta$ myo5 $\Delta$ strains more than 12 -fold. Vice versa, percentage of cells with a bipolar budding pattern dropped from $81 \%$ down to $39 \%$ in the myo3 $\Delta$ myo3 $\Delta$ and to $28 \%$ in the myo5 $\Delta$ myo5 $\Delta$ strain, respectively. Thus, expression of Myo3p or Myo5p alone is not sufficient to maintain a normal bipolar budding pattern suggesting that a certain levels of type I myosin is required for efficient selection of the cell poles as division sites. In recent study, it has been found that myosin I is required for hyphae formation in Candida albicans 
(Oberholzer et al., 2002). Therefore, we tested whether myosins I induce pseudohyphal development under nitrogen starvation conditions in yeast. In contrast, single mutant strains of $M Y O 3$ or MYO5 did not any effect in pseudohyphal induction (data not shown). This observation suggests that type I myosins are not required for filamentous development in S. cerevisiae.

A

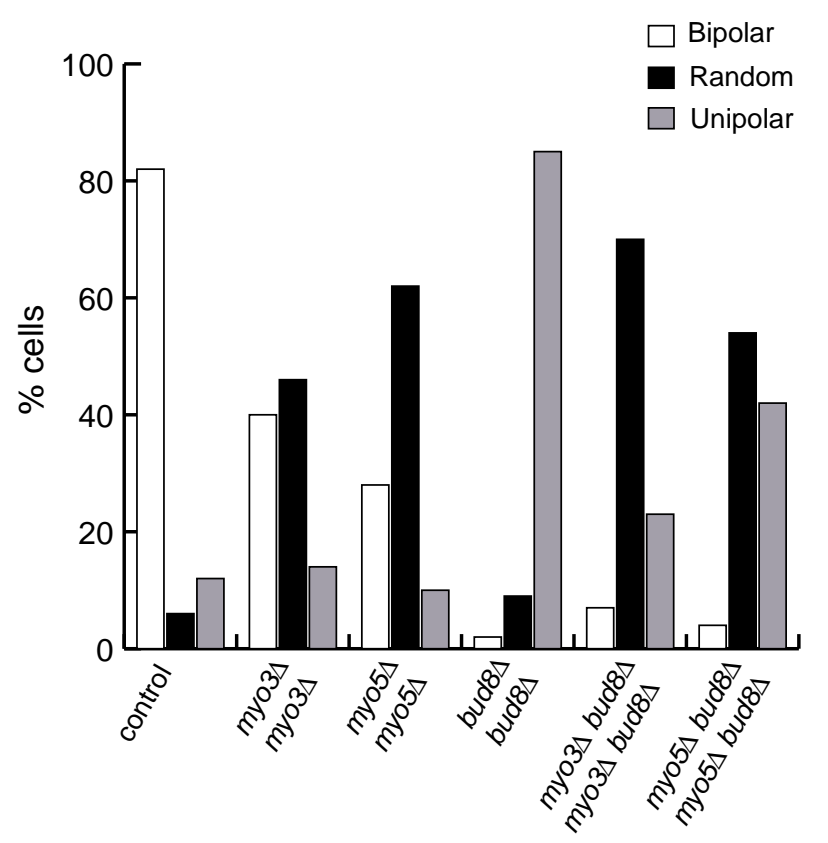

B
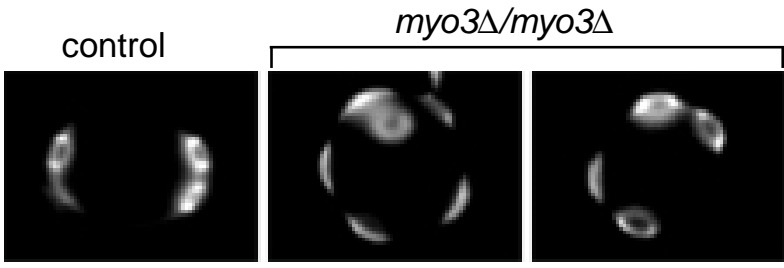

$m y 05 \Delta / m y 05 \Delta$

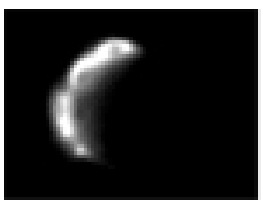

bud8s/bud8s

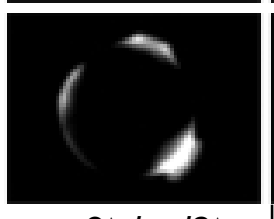

myo3 3 bud8s myo3s bud8s

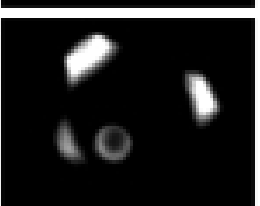

myo5 $\Delta$ bud8 8 myo5s bud8s

Figure 17. Deletion of $M Y O 3$ or $M Y O 5$ induces a random bud site selection pattern in diploid yeast cells. (A) Quantitative analysis of bud scar distribution. At least 200 cells of strains RH2447 (wild-

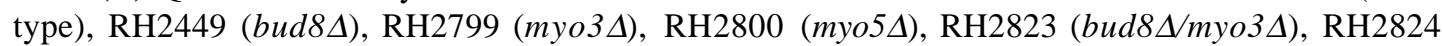
(bud8 8 myo5 $\Delta$ ) were analysed for bud scar distribution. Bars represent the percentage of cells exhibiting a bipolar (white bars), random (black bars) or unipolar (gray bars) budding pattern. (B) Budding patterns of diploid mutants were stained with Calcofluor. Strains, described in (A) grown to exponential phase on YPD media before staining with Calcofluor. Scale bar represents $5 \mu \mathrm{m}$. 
Mutations in $M Y O 3$ and $M Y O 5$ do not suppress asymmetric localization of Bud8p and Bud9p and are epistatic to $b u d 8 \Delta$ and $b u d 9 \Delta$ mutations

In our study, we found that myo3 $\Delta$ myo $3 \Delta$ and myo5 $\Delta$ myo5 $\Delta$ mutant strains resulted in significantly enhanced random budding pattern. These mutants might fail to deposit landmark proteins at the cell poles, as found in the case of other actinassociated proteins such as Bnilp. A second possibility is that type I myosins are more directly involved in the bud site selection pathway, e.g. in recongition of polar landmarks or in a later step involved in directing actin polarization towards the landmarks. To distinguish between these possibilities we determined the localization of GFP-Bud8p and GFP-Bud9p fusion proteins in strains lacking Myo3p or Myo5p. We found no differences in localization of GFP-Bud8p or GFP-Bud9p between a control strain and strains lacking either MYO3 or MYO5 (Figure 18).

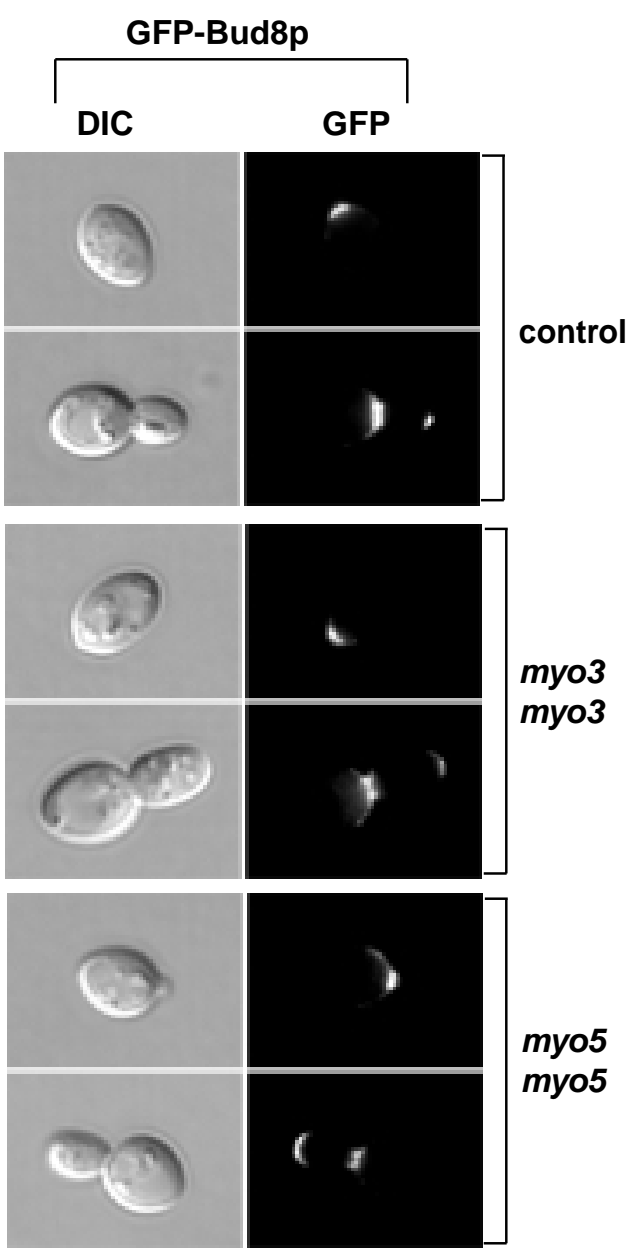

Figure 18. Localization of GFP-Bud8p is in the absence of Myo3p or Myo5p proteins. Living cells of

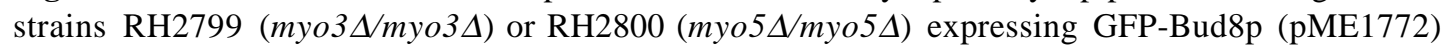
were grown on YNB-media to early exponential phase. Cells were viewed by DIC or by fluorescence microscopy (GFP). Scale bar represents $5 \mu \mathrm{m}$. 
Thus, random budding pattern of myo3 and myo5 mutants is not likely to be caused due to failure to deliver landmark proteins Bud8p and Bud9p to their site of action. Rather, type I myosin might act in the bud site selection pathway downstream of Bud8p and Bud9p. To further examine this possibility we constructed homozygous diploid myo3 and myo5 mutant in combination with mutations in BUD8 and BUD9. Mutants lacking $B U D 8$ are suppressed for selection of the distal pole and exhibit a unipolar proximal budding pattern, whereas bud9 $\Delta$ mutants fail to recognize the proximal pole and initiate budding with high frequency from the distal pole. Therefore, if strains lacking Myo3p or Myo5p have a random budding pattern because they fail to polarize the actin cytoskeleton towards the position of the landmark, one would expect mutations in $M Y O 3$ or MYO5 to be epistatic over bud8 and bud9 mutations. Indeed, we found that the budding pattern of the homozygous myo3 $\Delta$ bud $8 \Delta$ and myo5 $\Delta$ bud $8 \Delta$ more closely resembled the myo3 and myo5 single mutants and art of the bud8 mutant strain (Figure 17). Similar results were obtained with the bud9 mutants (data not shown).

Thus, type I myosins Myo3p and Myo5p do not appear to control delivery of bipolar landmark proteins to the cell poles, but might be involved in linking the actin cytoskeleton towards the spatial cues.

\section{Myo3p in vivo associates with Bud8p, but not with Bud9p}

We characterized the physical interactions between type I myosins and bipolar landmarks Bud8p and Bud9p. For this purpose, an in-frame fusion between the glutathione S-transferase gene (GST) and $M Y O 3$ was constructed and co-expressed together with myc epitope-tagged versions of either BUD8 or BUD9. GST-fusion proteins were affinity-purified with glutathione beads under conditions that allow copurification of associated proteins. Purified proteins were analyzed by Western blot analysis with either anti-GST or anti-myc antibodies (Figure 19). We found that under the conditions tested, myc-Bud9p could not be co-purified neither with GST alone nor with GST fused to Myo3p. However, significant amounts of myc-Bud8p could be co-purified with GST-Myo3p, when compared to the GST control. Thus, Myo3p appears to be a physical link between the actin cytoskeleton and the landmark protein Bud8p, suggesting that type I myosins might contribute to direct actin patches and zones of cell growth to the distal cell pole. 


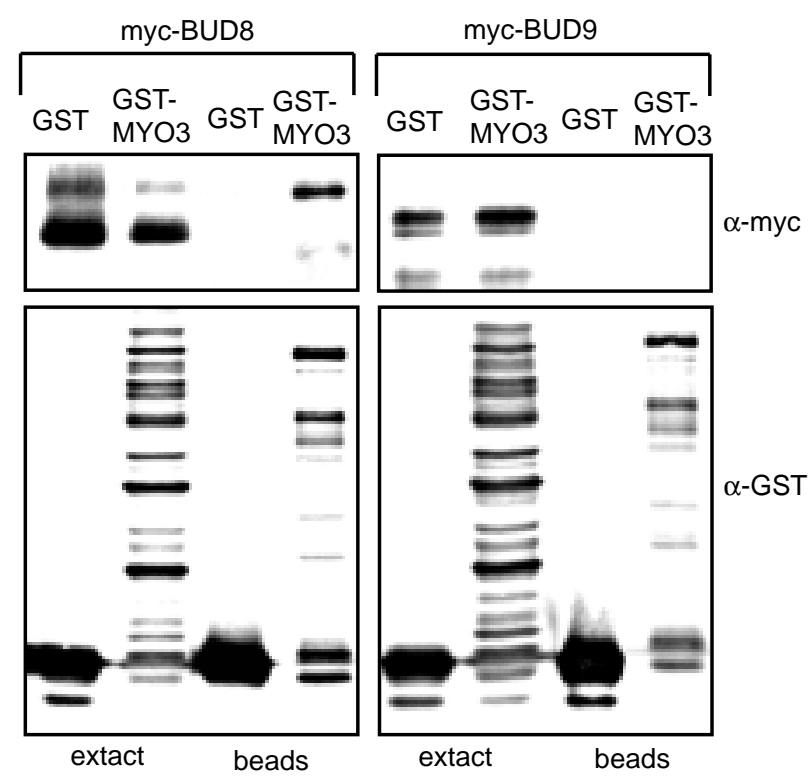

Figure 18. In vivo association of Myo3p with Bud8p. Total protein extracts were prepared from strain RH2495 carrying either of the plasmid pairs pME1937 (myc-BUD8) and pYGEX-2T (GST), pME1937 and pME2419 (GST-MYO3), pME1939 (myc-BUD9) and pYGEX-2T, or pME1939 and pME2419. GST and GST-fusion proteins were purified as described (Roberts et al., 1997). Equal amounts of each samples before (extract) and after (beads) affinity purification with glutathione agarose beads were subjected to SDS-PAGE, transferred to nitrocellulose membrane and blotted with a monoclonal anti-myc antibody $(\alpha$-myc) or a polyclonal anti-GST antibody $(\alpha-G S T)$.

\section{Discussion}

Here, we investigated how type I myosins affect selection of bud sites in $S$. cerevisiae. We find that both Myo3p and Myo5p are required for a regular bud site selection pattern, because expression of only Myo3p or Myo5p lead to a strong random phenotype. We provide genetic and biochemical evidence indicating that type I myosins might interact with the landmark protein Bud8p to control directed cell division. However, Myo3p and Myo5p, unlike other actin-regulating proteins, do not appear to be required for delivery of Bud8p to the distal pole. Thus, one interpretation of our data is that interaction of type I myosins with Bud8p might build the molecular basis that causes cortical actin patches to be concentrated to specific sites at the cell surface (e.g. the distal cell pole) and that induces site-specific (apical) cell growth. Previous studies have shown that Myo3p and Myo5p are associated with cortical actin patches at the zones of cells growth, where they might be directly involved in the promotion of actin assembly (Evangelista et al., 2000; Lechler et al., 2000; Pruyne and Bretscher, 2000). It has been suggested that type I myosins are 
involved in local nucleation of actin assembly at the sites of polarization (Lechler $e t$ al., 2001). This function appears to be regulated by Cdc42p and two classes of its effector proteins, the formin proteins Bnilp and Bnrlp and the PAK-like protein kinases Ste20p and Cla4p (Daniels and Bokoch, 1999; Lechler et al., 2001; Wu et $a l .$, 1997). The formin proteins have been suggested to be involved in the recruitment of type I myosins to the site of polarization, whereas the PAK-like kinase are thought to phosphorylate and activate the motor activity of type I myosins (Evangelista et al., 2000; Lechler et al., 2001; Lechler et al., 2000; Wu et al., 1997). In this context, it is very intriguing that Ste20p has been linked not only to type I myosins but also to Bud8p. Cells lacking Ste20p are unipolar proximal and Ste20p interacts with Bud8p in the two-hybrid system (Drees et al., 2001; Ni and Snyder, 2001; Sheu et al., 2000). Thus, Bud8p might affect type I myosin function. The localization of Bud8p appears to be actin-dependent, while it has been shown previously that Bud9p localization is both actin- and septin-dependent (Harkins et al., 2001; Ni and Snyder, 2001; Schenkman et al., 2002). The formin protein, Bnilp might be essential for localization of Bud8p at the distal pole of daughter cells, because Bud8p could not detected in bnil $\Delta b n i 1 \Delta$ mutant strains. Recently, it was reported that Bnilp interacts with both proteins, Myo3p and Myo5p (Evangelista et al., 2000; Johnson, 1999). However, our observations indicate that the localization of Bud8p is not altered in absence of each proteins, Myo3p or Myo5p. We could not test Bud8p localization in double mutant strain of $M Y O 3$ and MYO5, because double deletions of myosins I resulted in synthetic lethality in this study (data not shown). There are several possibilities that could explain our findings. Bud8p might help to recruit myosin to specific sites directly or indirectly, e.g. by interaction with $\mathrm{Cdc} 42 \mathrm{p}$ or the formins. Bud8p might affect interaction between myosin and PAK to regulate phosphorylation of the motor domains by the PAK leading to efficient nucleation of actin assembly at the distal pole. Additionally, Bud8p might influence other functions of myosins that have been previously discussed. Loss of both Myo3p and Myo5p causes severe growth defects and complete depolarization of actin patches or even invability depending on the genetic strain background (Geli and Riezman, 1996; Goodson et al., 1996). These studies suggest that type I myosins not only are required for polarized cell growth but also have a role that is essential for formation of the daughter cell. This essential function might be explained by the fact that Myo3p and Myo5p function have been associated with vesicular transport and endocytosis (Adams and Pollard, 1989a; Adams and Pollard, 1989b; Geli and Riezman, 1996; Goodson et al., 1996). Accumulation of intracellular membranes in the myo3 $\Delta$ and myo5 $\Delta$ double mutants suggested possible defects in membrane 
trafficking, previously (Goodson et al., 1996). Therefore, the motor proteins, Myo3p and Myo5p might be required for vesicle movement during endocytosis and secretion. Thus, Bud8p might be involved in these functions as well.

An interesting question is why Myo3p does not interact with Bud9p, yet type I myosins appear to be required for proximal bud site selection, owing the random budding phenotype of myo3 $\Delta$ and myo5 $\Delta$ mutants. This suggests that Bud9p might regulate proximal bud initiation by a mechanism that is slightly different from that used by Bud8p. One might imagine that Bud9p function also involves type I myosins but that the interaction involves a different set of other proteins.

In summary our study suggests that bud site selection, that is the direction of actin polarization and polarized cell growth to specific sites on the yeast cell surface, might be regulated by a mechanism that depends on the interaction of spatial landmark proteins with type I myosins.

Material and methods

\section{Construction of strains and plasmids}

All yeast strains used in this study are congenic to the $\Sigma 1278 \mathrm{~b}$ genetic background (Table IV). myo3 $\Delta::$ KanMX4 and myo5 $\Delta::$ KanMX4 deletion mutants were introduced using plasmids pME2420 and pME2421 (Table V). Double mutant strains carrying full deletions of $B U D 8$ or $B U D 9$ and $M Y O 3$ or $M Y O 5$ were constructed by genetic crosses. Standard methods for genetic crosses and transformation were used and standard yeast culture YPD, YNB and SC media were prepared essentially as described (Guthrie and Fink, 1991).

Plasmids pME2420 and pME2421 (Table V) carrying the myo3 $\Delta:: K a n R$ and myo5 $\Delta::$ KanR deletion cassettes were generated by PCR amplification using genomic DNA of corresponding strains from the EUROSCARF project (Winzeler $e t$ al., 1999). Amplified fragments were inserted into vector pBluescriptKS (Stratagene) to obtain pME2420 and pME2421 plasmids. Plasmid pME2419 was obtained by

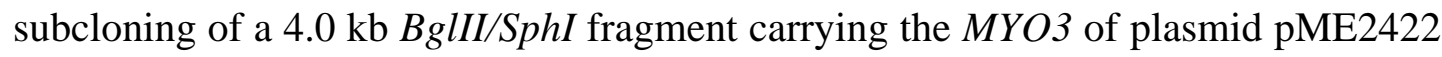
into BamHI/SphI site of plasmid pYGEX-2T (Schlenstedt et al., 1995) to obtain fusion between GST and MYO3. Plasmid pME2422 was constructed by PCR amplification of the MYO3 open reading frame from genomic DNA using primers MYO3X-6 (5'-GCGCGCGCGGCCGCCCACAGCGCCCATTCGGGAC-3') and MYO3X-7 (5'-CCGGAATTCCGGGGAGGGTTTACTGTGCTTTCTC-3') and 
subsequent cloning of the amplified $4.6 \mathrm{~kb} \mathrm{MYO3-fragment} \mathrm{into} \mathrm{vector}$ pBluescriptKS using restriction enzymes EcoRI and NotI.

\section{GST Pulldown}

Cells expressing a GST fusion protein together with myc-tagged versions of Bud8p or Bud9p were grown on YNB medium containing $2 \%$ glucose for $4 \mathrm{~h}$. After induction with $2 \%$ galactose for $3 \mathrm{~h}$, extracts were prepared as previously described (Roberts et al., 1997). Cells were chilled for $10 \mathrm{~min}$ on ice and harvested by centrifugation at $3000 \mathrm{rpm}$ at $4{ }^{\circ} \mathrm{C}$, after washing with buffer B $(50 \mathrm{mM}$ Tris-Cl pH 7.5, $100 \mathrm{mM} \mathrm{NaCl}, 5 \mathrm{mM}$ EDTA). Cells were resuspended in buffer B with complete protease inhibitor cocktail, PIM (Roche, Mannheim, Germany). Cells were after adding $0.1 \%$ TritonX-100 and $10 \%$ glycerol brocken using glass beads. Protein extracts were incubated with glutathione-agarose overnight at $4{ }^{\circ} \mathrm{C}$, and beads were repeatedly washed and collected to purify GST fusions and any associated proteins. Samples were denatured by heating at $60^{\circ} \mathrm{C}$ for $10 \mathrm{~min}$ in SDS sample buffer and equal amounts of each samples were subjected to SDS-PAGE and transferred to nitrocellulose membranes. Proteins fused to the myc-epitope tag were detected using ECL technology (Amersham, UK) after incubation of membranes with a mouse monoclonal anti-myc antibody (9E10) and a peroxidase-coupled goat anti-mouse secondary antibody (Dianova, Hamburg, Germany). GST fusion protein was detected using a rabbit polyclonal anti-GST antibody (Santa Cruz Biotechnologies, CA) and a peroxidase-coupled goat anti-rabbit IgG secondary antibody (Dianova, Hamburg, Germany).

\section{Fluorescence microscopy by GFP}

Yeast strains carrying plasmids encoding GFP-Bud8p or GFP-Bud9p were grown to early exponential phase in liquid YNB medium. Cells from $1 \mathrm{ml}$ of the cultures were harvested by centrifugation and immediately viewed in vivo on a Zeiss Axiovert microscope by either differential interference contrast microscopy (DIC) or fluorescence microscopy using a GFP filter set (AHF Analysentechnik AG, Tübingen, Germany). Cells were photographed using a Hamamatsu Orca ER digital camera and the Improvision Openlab software (Improvision, Coventry, UK).

\section{Bud scar staining and analysis of budding pattern}

Cells in exponential phase were prepared by growing strains on liquid YPD medium at $30{ }^{\circ} \mathrm{C}$ to an $\mathrm{OD}_{600}$ of 0.6 . After centrifugation, cells were fixed at room 
temperature for $2 \mathrm{~h}$ in $3.7 \%$ formaldehyde. Samples were rinsed twice in $\mathrm{H}_{2} \mathrm{O}$ and resuspended in $200 \mu \mathrm{l}$ of a fresh stock of $1 \mathrm{mg} / \mathrm{ml}$ calcofluor white (Fluorescent Brightener F-6259; Sigma). Bud scars were visualized by fluorescence microscopy using a Hamamatsu Orca ER digital camera and the Improvision Openlab software (Improvision, Coventry, UK). Cells with 2-10 obvious bud scars were divided into three classes: bipolar, random and unipolar bud site selection patterns. The percentage of cells represent each class for sample of at least 200 cells.

Table IV. Strains used in this work

\begin{tabular}{|c|c|c|}
\hline Strain & Genotype & Source \\
\hline RH2447 & 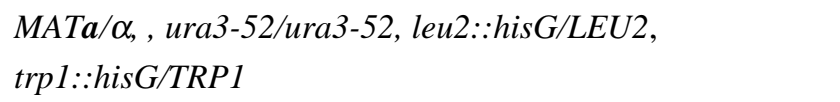 & Taheri et al., 2000 \\
\hline RH2449 & 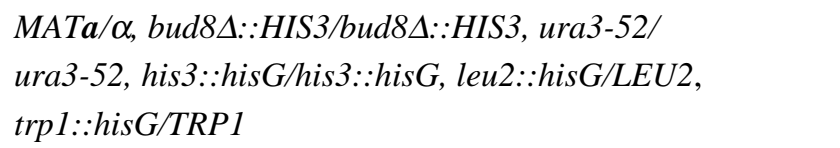 & Taheri et al., 2000 \\
\hline RH2495 & $\begin{array}{l}\text { MATa/MAT } \alpha, \text { ura3-52/ura3-52, leu2::hisG/leu2::hisG, } \\
\text { his3::hisG/HIS3, trp1::hisG/TRP1 }\end{array}$ & Taheri et al., 2000 \\
\hline RH2799 & 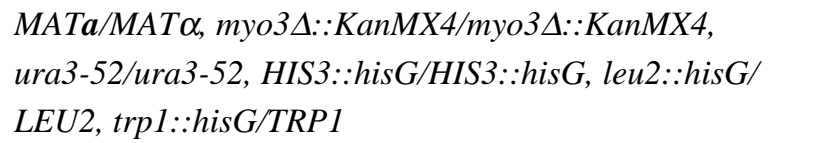 & this work \\
\hline RH2800 & 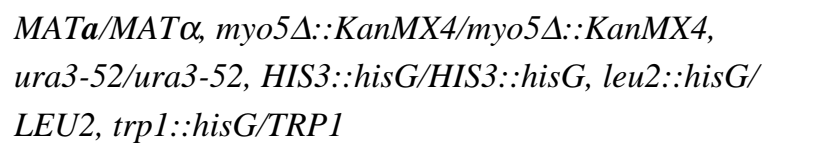 & this work \\
\hline RH2823 & 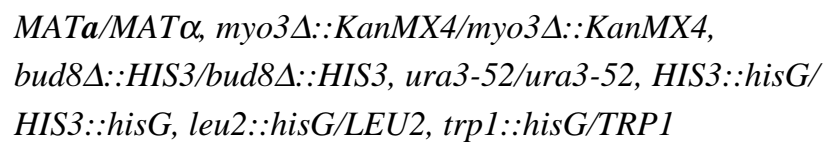 & this work \\
\hline RH2824 & 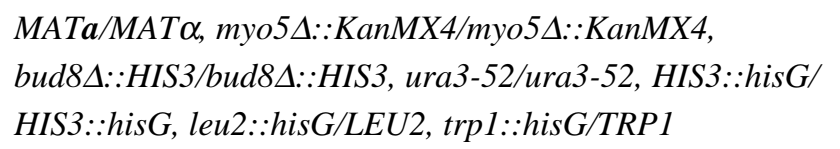 & this work \\
\hline RH2844 & 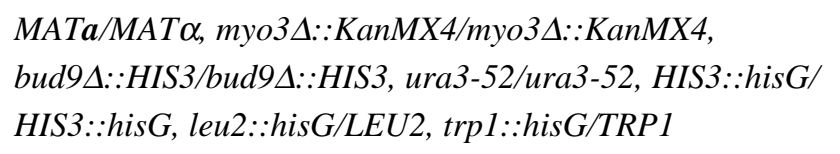 & this work \\
\hline RH2845 & 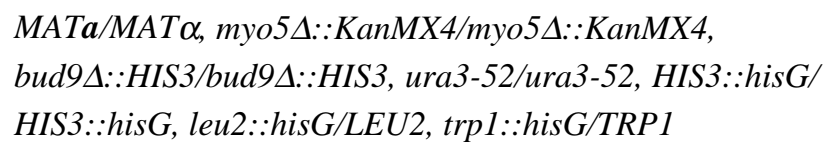 & this work \\
\hline
\end{tabular}


Table V. Plasmids used in this work

\begin{tabular}{lll}
\hline Plasmid & Discription & Reference \\
\hline pME1772 & BUD8prom-GFP-BUD8 fusion in pRS426 & Taheri et al., 2000 \\
pME1777 & BUD9prom-GFP-BUD9 fusion in pRS426 & Taheri et al., 2000 \\
pME1937 & BUD8prom-myc -BUD8 fusion in pRS425 & Taheri et al., 2000 \\
pME1939 & BUD9prom-myc -BUD9 fusion in pRS425 & Taheri et al., 2000 \\
pYGEX-2T & URA3-marked 2 $\mu$ m GAL1 prom-GST fusion vector & Schlentedt et al., 1995 \\
pME2419 & GAL1prom-GST-MYO3 fusion pYGEX-2T & this work \\
pME2420 & myo3 $::$ KanMX4 cassette for full deletion of $M Y O 3$ & this work \\
pME2421 & myo5 $::$ KanMX4 cassette for full deletion of $M Y O 5$ & this work \\
pME2422 & 4.6 kb fragment containing $M Y O 3$ in pBluescript & this work \\
& & \\
\hline
\end{tabular}




\section{References}

Adams, R.J. and Pollard, T.D. (1989a) Binding of myosin I to membrane lipids. Nature, 340, 565-568.

Adams, R.J. and Pollard, T.D. (1989b) Membrane-bound myosin-I provides new mechanisms in cell motility. Cell. Motil. Cytoskeleton, 14, 178-182.

Bähler, J. and Peter, M. (2000) Cell polarity in yeast. In Drubin, D.G. (ed.) Cell polarity. Oxford University Press, Oxford, Vol. 28, pp. 21-77.

Chant, J. (1996) Septin scaffolds and cleavage planes in Saccharomyces. Cell, 84, 187-190.

Chant, J. (1999) Cell polarity in yeast. Annu. Rev. Cell Dev. Biol., 15, 365-391.

Daniels, R.H. and Bokoch, G.M. (1999) p21-activated protein kinase: a crucial component of morphological signaling? Trends Biochem. Sci., 24, 350-355.

Drees, B.L., Sundin, B., Brazeau, E., Caviston, J.P., Chen, G.C., Guo, W., Kozminski, K.G., Lau, M.W., Moskow, J.J., Tong, A., Schenkman, L.R., McKenzie, A., 3rd, Brennwald, P., Longtine, M., Bi, E., Chan, C., Novick, P., Boone, C., Pringle, J.R., Davis, T.N., Fields, S. and Drubin, D.G. (2001) A protein interaction map for cell polarity development. J. Cell Biol., 154, 549-571.

Evangelista, M., Klebl, B.M., Tong, A.H., Webb, B.A., Leeuw, T., Leberer, E., Whiteway, M., Thomas, D.Y. and Boone, C. (2000) A role for myosin-I in actin assembly through interactions with $\operatorname{Vrp} 1 \mathrm{p}$, Bee1p, and the Arp2/3 complex. $J$. Cell Biol., 148, 353-362.

Geli, M.I. and Riezman, H. (1996) Role of type I myosins in receptor-mediated endocytosis in yeast. Science, 272, 533-535.

Goodson, H.V., Anderson, B.L., Warrick, H.M., Pon, L.A. and Spudich, J.A. (1996) Synthetic lethality screen identifies a novel yeast myosin I gene (MYO5): myosin I proteins are required for polarization of the actin cytoskeleton. J. Cell Biol., 133, 1277-1291.

Guthrie, C. and Fink, G.R. (1991) Guide to Yeast Genetics and Molecular Biology. Methods Enzymol., 194.

Harkins, H.A., Page, N., Schenkman, L.R., De Virgilio, C., Shaw, S., Bussey, H. and Pringle, J.R. (2001) Bud8p and Bud9p, proteins that may mark the sites for bipolar budding in yeast. Mol. Biol. Cell, 12, 2497-2518.

Johnson, D.I. (1999) Cdc42: An essential Rho-type GTPase controlling eukaryotic cell polarity. Microbiol. Mol. Biol. Rev., 63, 54-105.

Kang, P.J., Sanson, A., Lee, B. and Park, H.O. (2001) A GDP/GTP exchange factor involved in linking a spatial landmark to cell polarity. Science, 292, 1376-1378.

Lechler, T., Jonsdottir, G.A., Klee, S.K., Pellman, D. and Li, R. (2001) A two-tiered mechanism by which Cdc42 controls the localization and activation of an Arp2/3activating motor complex in yeast. J. Cell Biol., 155, 261-270. 
Lechler, T., Shevchenko, A. and Li, R. (2000) Direct involvement of yeast type I myosins in Cdc42-dependent actin polymerization. J. Cell Biol., 148, 363-373.

Marston, A.L., Chen, T., Yang, M.C., Belhumeur, P. and Chant, J. (2001) A localized GTPase exchange factor, Bud5, determines the orientation of division axes in yeast. Curr. Biol., 11, 803-807.

Ni, L. and Snyder, M. (2001) A Genomic Study of the Bipolar Bud Site Selection Pattern in Saccharomyces cerevisiae. Mol. Biol. Cell, 12, 2147-2170.

Oberholzer, U., Marcil, A., Leberer, E., Thomas, D.Y. and Whiteway, M. (2002) Myosin I Is Required for Hypha Formation in Candida albicans. Eukaryot. Cell, 1, 213-228.

Palmieri, S.J. and Haarer, B.K. (1998) Polarity and division site specification in yeast. Curr. Opin. Microbiol., 1, 678-686.

Park, H.O., Sanson, A. and Herskowitz, I. (1999) Localization of Bud2p, a GTPaseactivating protein necessary for programming cell polarity in yeast to the presumptive bud site. Genes Dev., 13, 1912-1917.

Pringle, J.R., Bi, E., Harkins, H.A., Zahner, J.E., De Virgilio, C., Chant, J., Corrado, K. and Fares, H. (1995) Establishment of cell polarity in yeast. Cold Spring. Harb. Symp. Quant. Biol., 60, 729-744.

Pruyne, D. and Bretscher, A. (2000) Polarization of cell growth in yeast. II. The role of the cortical actin cytoskeleton. J. Cell Sci., 113, 571-585.

Roberts, R.L., Mösch, H.-U. and Fink, G.R. (1997) 14-3-3 proteins are essential for RAS/MAPK cascade signaling during pseudohyphal development in S. cerevisiae. Cell, 89, 1055-1065.

Roemer, T., Madden, K., Chang, J. and Snyder, M. (1996) Selection of axial growth sites in yeast requires Axl2p, a novel plasma membrane glycoprotein. Genes Dev., 10, 777-793.

Schenkman, L.R., Caruso, C., Page, N. and Pringle, J.R. (2002) The role of cell cycle-regulated expression in the localization of spatial landmark proteins in yeast. J. Cell Biol., 156, 829-841.

Schlenstedt, G., Saavedra, C., Loeb, J.D., Cole, C.N. and Silver, P.A. (1995) The GTP-bound form of the yeast Ran/TC4 homologue blocks nuclear protein import and appearance of poly(A)+ RNA in the cytoplasm. Proc. Natl. Acad. Sci. U.S.A., 92, 225-229.

Sheu, Y.J., Barral, Y. and Snyder, M. (2000) Polarized growth controls cell shape and bipolar bud site selection in Saccharomyces cerevisiae. Mol. Cell. Biol., 20, 5235-5247.

Snyder, M. (1989) The Spa2p protein of yeast localizes to sites of cell growth. J. Cell Biol., 108, 1419-1429.

Taheri, N., Köhler, T., Braus, G.H. and Mösch, H.-U. (2000) Asymmetrically localized Bud8p and Bud9p proteins control yeast cell polarity and development. EMBO J., 19, 6686-6696. 
Winzeler, E.A., Shoemaker, D.D., Astromoff, A., Liang, H., Anderson, K., Andre, B., Bangham, R., Benito, R., Boeke, J.D., Bussey, H., Chu, A.M., Connelly, C., Davis, K., Dietrich, F., Dow, S.W., El Bakkoury, M., Foury, F., Friend, S.H., Gentalen, E., Giaever, G., Hegemann, J.H., Jones, T., Laub, M., Liao, H., Davis, R.W. and et al. (1999) Functional characterization of the $S$. cerevisiae genome by gene deletion and parallel analysis. Science, 285, 901-906.

Wu, C., Lytvyn, V., Thomas, D.Y. and Leberer, E. (1997) The phosphorylation site for Ste20p-like protein kinases is essential for the function of myosin-I in yeast. $J$. Biol. Chem., 272, 30623-30626.

Zahner, J.E., Harkins, H.A. and Pringle, J.R. (1996) Genetic analysis of the bipolar pattern of bud site selection in the yeast Saccharomyces cerevisiae. Mol. Cell. Biol., 16, 1857-1870. 


\title{
Chapter 4
}

\section{The spatial landmark protein Bud8p links yeast cell polarity to factors involved in cellular transport and translation}

\begin{abstract}
In Saccharomyces cerevisiae, Bud8p is a transmembrane glycoprotein that appears to mark the distal cell pole as site for cell division. Bud8p is highly concentrated at the distal pole of both single yeast form (YF) cells and of pseudohyphal (PH) cells growing as linear filaments. Here, we performed a structural and functional analysis of Bud8p. A systematic deletion analysis revealed novel variants of Bud8p that induce unipolar distal or random budding patterns. Two-hybrid screening identified several cytoplasmically localized proteins that interact with the $\mathrm{N}$-terminal part of Bud8p and that have been implicated in cellular transport and translation. Two of these proteins, Rpl12p and Scp160p, associate with Bud8p in vivo, interactions which are independent of the two transmembrane domains that are required for distal pole localization of Bud8p. Because the Bud8p-interacting proteins identified here are also required for appropriate budding patterns, we suggest that Bud8p might regulate cell polarity and directed cell division by interaction with components that have essential functions in the processes of cellular transport and translation.
\end{abstract}




\section{Introduction}

Establishment and maintenance of cell polarity is fundamental for growth and development of eukaryotic and prokaryotic organisms and is manifested by asymmetric cellular distribution of proteins, nucleic acids, macromolecular assemblies and organelles (Ni and Snyder, 2001). The budding yeast Saccharomyces cerevisiae is an useful model to study various aspects of cell polarization, because this organism establishes polarity at several stages during division and development (Chant, 1999; Madden and Snyder, 1998; Pruyne and Bretscher, 2000a). Establishment of yeast cell polarity can be divided into three basic steps (for reviews see (Bähler and Peter, 2000; Drubin, 1991; Drubin and Nelson, 1996; Pruyne and Bretscher, 2000b). First, cells choose a site on their surface, a spatial 'landmark', towards which they will polarize. In a second step, the landmark is recognized by a number of proteins, that collectively are called polarity establishment proteins or actin-organizing complex. In a final step, this complex of proteins recruits the machinery that organizes and polymerizes the actin cytoskeleton to the site of the landmark. As a consequence, cell wall and other materials are transported along the polarized actin cytoskeleton towards the landmark, leading to directed cell division and polarized growth.

At the beginning of every round of cell division, yeast cells must choose the site for bud initiation, which is not a random process, but follows specific spatial patterns that are under genetic control of the corresponding cell type (Chant and Pringle, 1995; Freifelder, 1960; Hicks et al., 1977). Haploid cells bud in an axial pattern, where mother and daughter cells bud adjacent to the cell pole that defined the previous mother-daughter junction. This region of the yeast cell surface is also referred to as the proximal pole or the birth end of the cell. Diploid yeast cells bud in a bipolar pattern, where buds form either at the proximal pole or at the site opposite to it, called the distal pole. In addition to genetic control, yeast cell polarity and corresponding budding patterns are affected by extracellular stimuli, such as pheromones or nutrients (Madden and Snyder, 1992; Segall, 1993). For instance, diploid cells starved for nitrogen switch their budding pattern from bipolar to unipolar distal, where most of the cell divisions are initiated at the distal cell pole (Gimeno et al., 1992; Kron et al., 1994).

Genetic analysis has identified a large number of genes that are involved in the process of bud site selection, which initially have been classified according to their loss-of-function phenotype. One class of genes is specifically required for axial budding of haploid cells without affecting the bipolar pattern of diploids and includes 
BUD3, BUD4, AXL2/BUD10 and AXL1 (Chant and Herskowitz, 1991; Fujita et al., 1994; Halme et al., 1996; Roemer et al., 1996). Bud3p, Bud4p and Axl2p/Bud10p together with septins appear to constitute a transient landmark that is deposited at the mother-bud neck during each cell cycle (Lord et al., 2000; Sanders and Herskowitz, 1996).

A second class of genes is required for axial and bipolar budding and includes RSR1/BUD1, BUD2 and BUD5 (Bender and Pringle, 1989; Chant et al., 1991; Chant and Pringle, 1991). Mutations in these genes cause random budding patterns in haploid and diploid cells. Rsr1p/Bud1p, Bud2p and Bud5p constitute a GTPase signaling module that is thought to help direct bud formation components to the site of cell division by interaction with the spatial landmark (Park et al., 1997; Park et al., 1993; Park et al., 1999). Physical interaction has been found between the guanine nucleotide exchange factor Bud5p and the haploid-specific axial landmark protein Ax12p/Bud10p (Kang et al., 2001).

A third class of more than 100 genes is required for the bipolar pattern of diploid yeast but not for the axial pattern. Mutations in these genes either shift the bud site selection pattern of diploid cells from bipolar to unipolar (with bias to either the distal or the proximal pole) or they cause random budding (Bauer et al., 1993; Chen et al., 2000; Mösch and Fink, 1997; Ni and Snyder, 2001; Sheu et al., 2000; Snyder, 1989; Zahner et al., 1996). Therefore, proteins of this class are likely to either be (i) bipolar landmark components, (ii) factors involved in the synthesis or polar transport of spatial cues, or (iii) downstream components that recognize landmark proteins and are recruited to the respective cell pole. (i) Potential bipolar landmark components include the two transmembrane glycoproteins Bud8p and Bud9p (Harkins et al., 2001; Taheri et al., 2000; Zahner et al., 1996). Bud8p is required for distal bud site selection and is localized to the distal cell pole, suggesting that is it part of the distal landmark. Bud9p is required for proximal pole selection and is localized at the proximal pole, indicating that it functions as part of the proximal tag (Harkins et al., 2001). Because Bud9p is also found at the distal pole and physically interacts with Bud8p, it might fulfill an additional function at the distal pole, where it seems to act as a nutritionally-controlled inhibitor of distal budding (Taheri et al., 2000). Rax2p is further membrane protein that appears to be part of the bipolar landmark (Chen et al., 2000). (ii) A genome-wide screen has identified a large number of genes that might be involved in the synthesis, transport or recognition of bipolar landmarks ( $\mathrm{Ni}$ and Snyder, 2001). Some of these proteins appear to affect the transport of Bud8p to the distal pole, including the actin cytoskeleton-associated proteins Bni1p, Spa2p and Bud6p or the t-SNARE protein Vam3p. Others are likely to be involved in posttrans- 
lational modification of landmark proteins. These factors include Pmt2p, a mannosyltransferase that is required for O-linked glycosylation of Bud8p, and Ste20p, a PAK-like protein kinase that acts in the same genetic pathway as Bud8p and interacts with Bud8p in the yeast two-hybrid system (Drees et al., 2001; Sheu et al., 2000). (iii) Factors that might be involved in recognition of diploid-specific landmarks, and therefore would act downstream of the spatial cues, include Bud5p. Polar localization of this general polarity factor depends on the presence of Bud8p and Bud9p (Kang et al., 2001). In contrast to the axial landmark protein Ax12p/Bud10p, however, no direct physical link has been established between Bud5p and Bud8p or Bud9p.

Although genetic and cell biological analysis has led to the identification of a large number of the components that constitute the bud site selection pathway in diploid yeast cells, the molecular function of individual factors in many cases is only poorly understood. For instance, the exact mechanism, by which the potential landmark proteins Bud8p and Bud9p regulate site-specific initiation of cell division is not known. The overall structure of Bud8p and Bud9p are similar in that both are predicted to consist of a large N-terminal extracellular domain, followed by a first membrane-spanning domain (TM1), a short cytoplasmic loop, a second membranespanning domain (TM2), and a very short extracellular domain at the C-terminus (Chant, 1999; Harkins et al., 2001; Taheri et al., 2000). The N-terminal portion of both proteins contain several $\mathrm{N}$ - and $\mathrm{O}$-glycosylation sites that appear to be functional (Harkins et al., 2001). The large N-terminal part of Bud8p also contains a putative RNA recognition motif (RRM) (Figure 20A) (Bandziulis et al., 1989) and three PEST-like sequences (Rechsteiner and Rogers, 1996), but the function of these domains is not known. Two-hybrid analysis has identified four putative Bud8pinteraction partners, Ste20p, Nup116p, Ybr027p and Yk1082p (Drees et al., 2001; Ito et al., 2001). However, these interactions have not been further corroborated by a biochemical analysis. For Bud9p, only Bud8p has been identified as an interaction partner that is associated in vivo (Taheri et al., 2000).

In order to better understand the structure and function of bipolar landmark proteins, we performed a detailed functional analysis of Bud8p. We find that the large $\mathrm{N}$ terminal portion of Bud8p carries several functional domains that differentially affect bud site selection. The $\mathrm{C}$-terminal transmembrane domain is required for localizing Bud8p to the distal cell pole. Two-hybrid screening and subsequent biochemical copurification experiments reveal that the N-terminal part of Bud8p specifically interacts with Rp112Ap, the 26S rRNA binding ribosomal protein L12 of $S$. cerevisiae (Briones et al., 1998; Pucciarelli et al., 1990), Scp160p, a multiple KH- 
domain protein and a component of mRNP complexes in yeast (Frey et al., 2001; Lang and Fridovich-Keil, 2000), and Trs120p, a part of the TRAPP (transport protein particle) complex that plays a key role in the late stages of endoplasmic reticulum to Golgi traffic in yeast (Sacher et al., 2000). We find that two of these proteins, Rpl12Ap and Scp160p, are required for appropriate bud site selection of diploid yeast cells, but not for distal localization of Bud8p. Our findings suggest that association of Bud8p with factors involved in translation and cellular transport might represent a mechanism, by which landmark proteins help to establish asymmetric distribution of multiprotein complexes or subcellular compartments and control directed cell division.

\section{Results}

\section{Systematic deletion analysis of Bud8p reveals several novel functional domains}

To get a better insight into the structure and function of Bud8p, a series of novel BUD8 alleles was constructed by systematic deletion analysis of a tagged version of $B U D 8\left(m y c^{6}-B U D 8\right)$ that carries an insertion of six copies of the $m y c$ epitope tag after the second codon and that is able to functionally replace the endogenous BUD8. Resulting Bud8p mutant proteins carry deletions of 37 to 152 amino acids in length, which span the whole protein in an overlapping manner (Figure 20A). Two further alleles were constructed that code for Bud8p mutant proteins lacking most of the $\mathrm{N}$ terminal part (Bud8 $\mathrm{p}^{\Delta 7-417}$ and Bud8 $\mathrm{p}^{\Delta 7-505}$ ).

To study the functionality of these novel Bud8p mutant proteins and to ensure expression at endogenous levels, diploid yeast strains were constructed containing two genomic copies of either of the epitope-tagged $B U D 8$ mutant alleles instead of the endogenous copies of $B U D 8$. With the exception of Bud8 $8 \mathrm{p}^{47-505}$, all epitopetagged Bud8p mutant proteins produced specific and detectable signals, when corresponding yeast strains were analyzed by Western analysis (Figure 20B). In our hands, myc ${ }^{6}$-tagged Bud8p (with a calculated molecular weight of $75.4 \mathrm{kD}$ ) reproducibly appeared as a multiple band pattern when separated by SDS polyacrylamide gel electrophoresis (Figure 20B). The lowest molecular form appeared around $85 \mathrm{kD}$, and several bands of higher molecular weight could be observed between $130 \mathrm{kD}$ and $140 \mathrm{kD}$. Appearance of Bud8p at a size higher than the calculated molecular weight was shown to result in part from glycosylation (Harkins et al., 2001). 
A
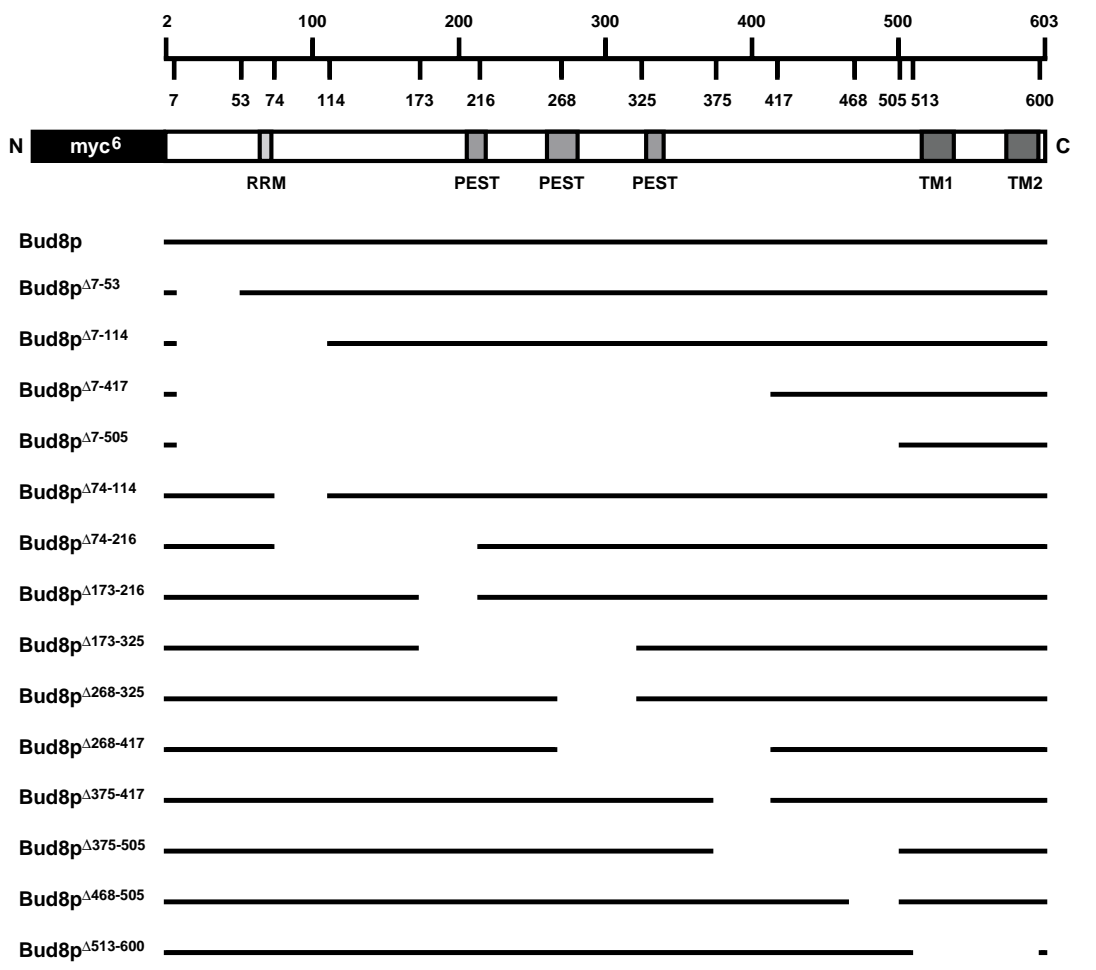

B

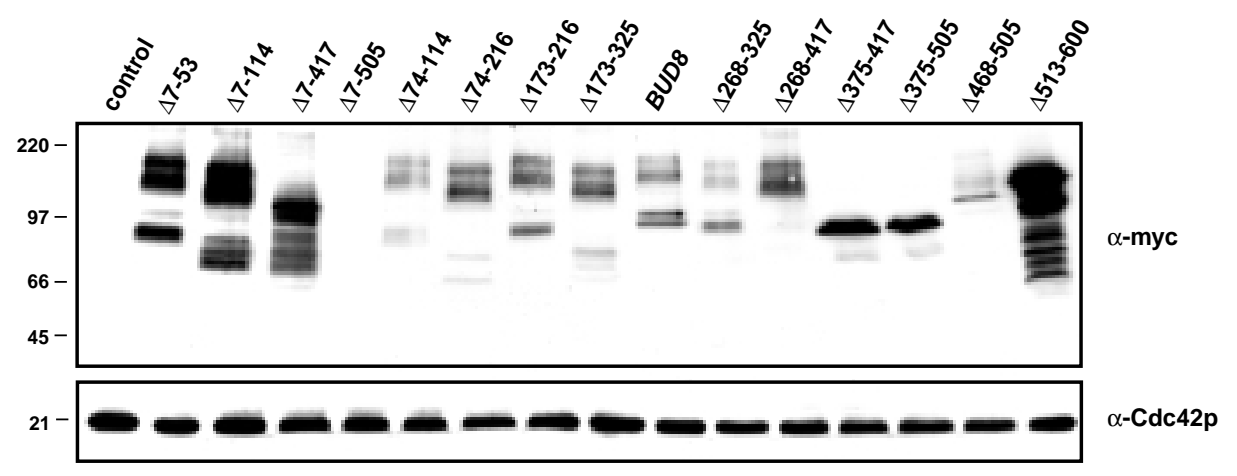

Figure 20. Systematic deletion analysis of Bud8p. (A) Structure of Bud8p and Bud8p mutant proteins. Numbers on the ruler correspond to the Bud8p amino acid sequence. Bottom numbers indicate the deletion endpoints. All proteins carry six copies of the myc epitope tag $\left(\mathrm{myc}^{6}\right)$ shown in black that is located between the first and the second codon at the N-terminus (N) of Bud8p. Positions of putative RNA-binding domain (RRM), three putative PEST sequences (PEST), and two membrane spanning domains (TM1 and TM2) close to the C-terminus (C) are indicated. Superscript numbers of Bud8p mutant proteins indicate the first and last amino acid residue of the deleted segments that are shown as omissions on the right side. (B) Expression levels of Bud8p proteins. Total protein extracts were prepared from strains expressing non-tagged $B U D 8$ (RH2782, control) , $m y c^{6}-B U D 8$ (RH2783, $B U D 8)$, and $m y c^{6}-B U D 8$ mutant alleles (RH2784, $\left.\Delta 7-53\right)$, (RH2785, $\left.\Delta 7-114\right)$, (RH2786, $\left.\Delta 7-417\right)$, (RH2787, $\Delta 7-505),(\mathrm{RH} 2788, \Delta 74-114),(\mathrm{RH} 2789, \Delta 74-216),(\mathrm{RH} 2790, \Delta 173-216),(\mathrm{RH} 2791, \Delta 173-$ 325), (RH2792, $\Delta 268-325),(\mathrm{RH} 2793, \Delta 268-417),(\mathrm{RH} 2794, \Delta 375-417)$, (RH2795, $\Delta 375-505)$, (RH2796, $\Delta 468-505)$, (RH2797, $\Delta 513-600)$. Extracts were analysed for expression of myc tagged proteins by Western blot analysis using a monoclonal anti-myc antibody $(\alpha-$ myc). As an internal control, expression of Cdc42p was measured using an anti-Cdc42p antibody (lower panel). Molecular size standards (in $\mathrm{kDa}$ ) are shown on the left side. 
Eleven out of the fourteen Bud8p mutant proteins also produced multiple bands, with one band appearing in the range of the calculated molecular weight and with further bands appearing at a higher size (Figure 20B). In contrast, the two mutant proteins Bud $8 \mathrm{p}^{\Delta 375-417}$ and Bud8 $\mathrm{p}^{\Delta 375-505}$ produced only a single band, with Bud8 $\mathrm{p}^{\Delta 375-417}$ appearing in the range of the calculated size and Bud8 $\mathrm{p}^{\Delta 375-505}$ appearing at a size higher than predicted.

Functionality of the different Bud8p mutant proteins was determined by analysis of the bud site selection patterns produced by the corresponding mutant strains. For this purpose, bud scar staining was performed on cells growing in the yeast-form (YF) (Figures 21A and 21B). In addition, time lapse microscopy was used, in order to distinguish between unipolar proximal (at the birth pole of the cell) and unipolar distal (at the opposite to the birth pole) budding patterns (Figure 21C and Table VI). Strains were further tested for filamentous pseudohyphal $(\mathrm{PH})$ development (Figure 22), because this growth phenotype is a good measure for the ability of strains to switch to the unipolar distal budding pattern when starved for nitrogen (Gimeno et al., 1992; Kron et al., 1994). As previously shown, diploid bud84bud84 deletion strains elaborated a unipolar proximal budding pattern and were suppressed for $\mathrm{PH}$ growth (Harkins et al., 2001; Taheri et al., 2000). A strain expressing the myc ${ }^{6}$ tagged full length version of $B U D 8$ was phenotypically indistinguishable from a strain expressing the non-tagged $B U D 8$ wild-type gene in that both produced a bipolar budding pattern in YF cells and were able to undergo regular $\mathrm{PH}$ development (Figure 21 A and Figure 22). Budding patterns produced by strains expressing the different Bud8p mutant proteins defined four distinct classes. The first class included seven mutants that carried the $B U D 8^{47-417}, B U D 8^{47-505}, B U D 8^{474-216}$, $B U D 8^{4375-417}, B U D 8^{4375-505}, B U D 8^{4468-505}$, or $B U D 8^{4513-600}$ alleles. These mutants resembled a strain carrying a full deletion of $B U D 8$ in that they selelcted the proximal pole for budding with very high frequency and were found to be suppressed for PH development (Figure 21, Figure 22, and Table VI). The second class was defined by a single mutant that expressed the $B U D 8^{474-114}$ allele. This strain produced a budding pattern similar to a BUD 8 wild-type strain. The third class included two mutants expressing either $B U D 8^{\Delta 7-53}$ or $B U D 8^{\Delta 7-114}$, which both code for variants of Bud8p that carry deletions close to the $\mathrm{N}$-terminus. Interestingly, these mutants budded with a significantly higher frequency from the distal pole (Figure 21 and Table (VI). 


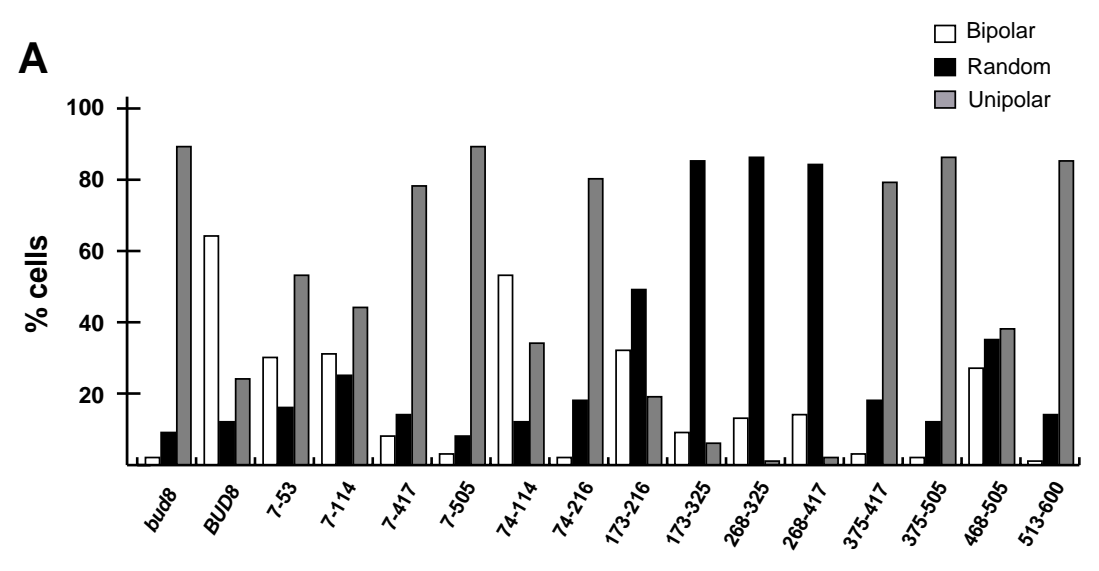

B

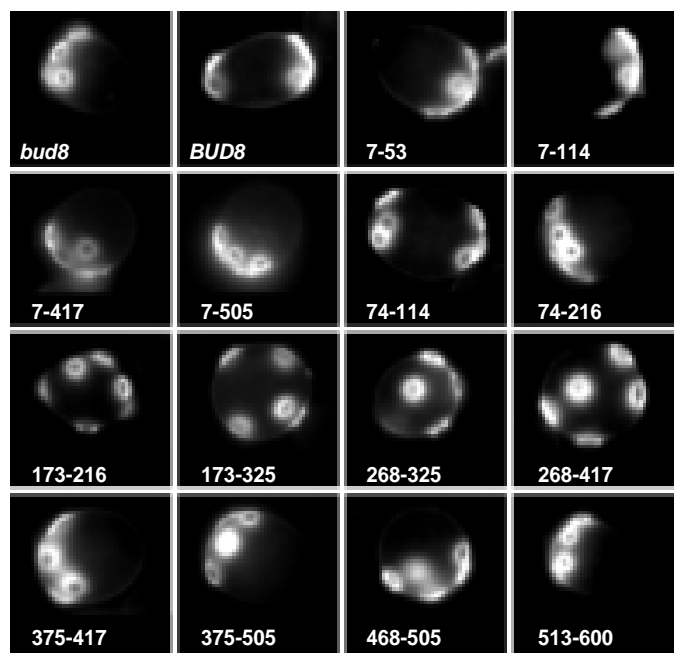

C

bud8A

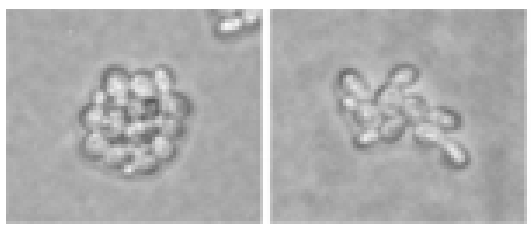

$\Delta 7-53$

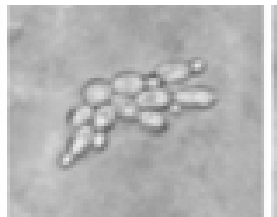

$\Delta 7-114$

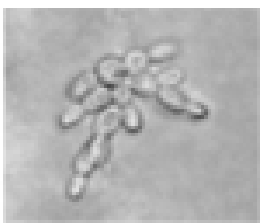

Figure 21. Regulation of bud site selection by Bud8p mutant proteins. (A) Quantitative analysis of

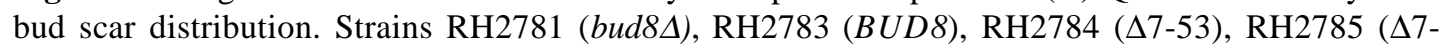
114), RH2786 ( $\Delta 7-417)$, RH2787 ( $\Delta 7-505)$, RH2788 ( $\Delta 74-114)$, RH2789 ( $\Delta 74-216)$, RH2790 $(\Delta 173-$

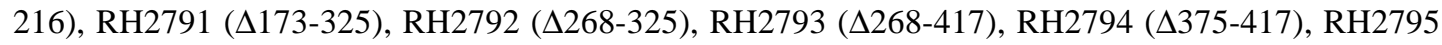
$(\Delta 375-505)$, RH2796 ( $\Delta 468-505)$, and RH2797 ( $\Delta 513-600)$ were grown to exponential phase, and at least 200 cells of each strain were analyzed for bud scar distribution after staining of bud scars with calcofluor. Bars represent the percentage of cells exhibiting a bipolar (white bars), random (black bars) or unipolar (gray bars) budding pattern. (B) Fluorescence imaging of representative cells of strains described in (A). Scale bar represents $5 \mu \mathrm{m}$. 
Table VI. Time lapse analysis of budding patterns induced by different $B U D 8$ alleles

\begin{tabular}{|c|c|c|c|}
\hline \multirow[t]{2}{*}{ Strain } & \multirow[t]{2}{*}{ BUD8 allele } & \multicolumn{2}{|c|}{ First bud of virgin daughter cells } \\
\hline & & $\%$ proximal & $\%$ distal \\
\hline RH2781 & bud84 & 74 & 26 \\
\hline RH2782 & BUD8 & 2 & 98 \\
\hline RH2783 & $m y c^{6}-B U D 8$ & 3 & 97 \\
\hline RH2784 & $m y c^{6}-B U D 8^{\Delta 7-53}$ & 0 & 100 \\
\hline RH2785 & $m y c^{6}-B U D 8^{\Delta 7-114}$ & 0 & 100 \\
\hline RH2786 & $m y c^{6}-B U D 8^{47-417}$ & 63 & 37 \\
\hline RH2787 & $m y c^{6}-B U D 8^{\Delta 7-505}$ & 64 & 36 \\
\hline RH2789 & $m y c^{6}-B U D 8^{\Delta 74-216}$ & 63 & 37 \\
\hline RH2794 & $m y c^{6}-B U D 8^{\Delta 375-417}$ & 69 & 31 \\
\hline RH2795 & $m y c^{6}-B U D 8^{4375-505}$ & 67 & 33 \\
\hline RH2797 & $m y c^{6}-B U D 8^{4513-600}$ & 72 & 28 \\
\hline
\end{tabular}

Enhanced distal budding has not been observed for mutations in BUD8 so far and has been found to be typical for strains that lack BUD9 (Harkins et al., 2001; Taheri et al., 2000). A fourth class included the $B U D 8^{\Delta 173-216}, B U D 8^{\Delta 173-325}, B U D 8^{\Delta 268-325}$ and $B U D 8^{4268-417}$ expressing strains. Surprisingly, these mutants produced a random budding pattern with very high frequency. Random budding has not been observed for $B U D 8$ mutants, except when $B U D 8$ is completely deleted together with $B U D 9$ (Taheri et al., 2000). Random budding is typical for mutations in general budding genes, such as RSR1/BUD1, BUD2 or BUD5, and for mutations affecting the actin cytoskeleton (Chant et al., 1991; Chant and Herskowitz, 1991; Ni and Snyder, 2001). In contrast to strains lacking $R S R 1 / B U D 1$ or double mutants lacking $B U D 8$ and $B U D 9$, randomly budding $B U D 8$ mutants identified here were still able to produce significant amounts of pseudohyphae, when nutritionally starved for nitrogen (Figure 22). Taken together, the systematic deletion analysis of Bud8p performed in this section allows assignment of distinct functions to several regions of the protein. The region including residues 7 to 53 appears to inhibit Bud8p from directing cell division to the distal pole, because deletion of this region shifts the budding pattern from bipolar to unipolar distal. Residues 216 to 373 that include the three putative PEST sequences might be responsible for proper interaction of Bud8p with factors involved in general polarity establishment, because Bud8p variants lacking this segment cause a general loss of cell polarity. The three regions encompassing residues 74-173, 417-468 and 513-600 are critical for Bud8p to fulfill any function at all, because their absence has phenotypic consequences that are identical to a complete absence of Bud8p. 
These segments might be responsible for transport of Bud8p to its site of action or for interaction of Bud8p with downstream acting factors.

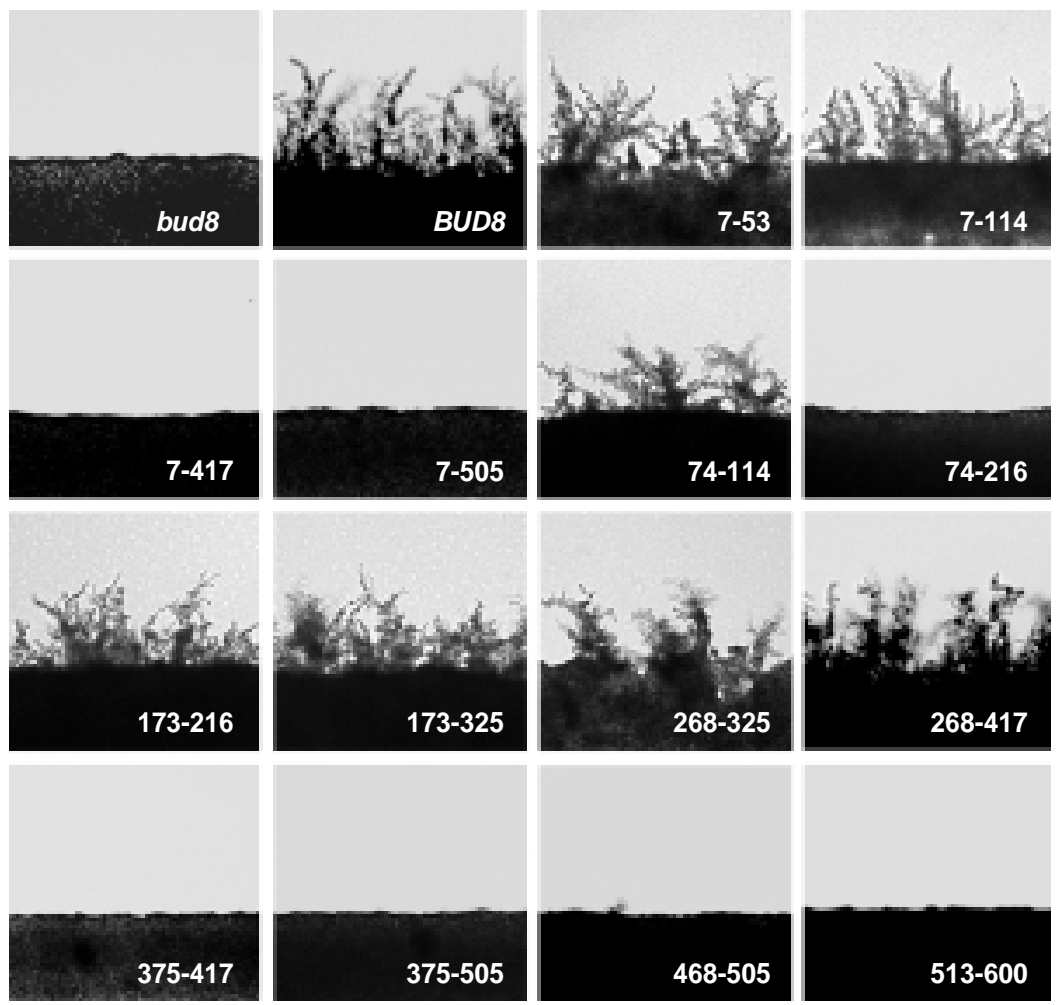

Figure 22. Pseudohyphal development of BUD8 deletion mutants. Strains RH2781 (bud84), RH2783

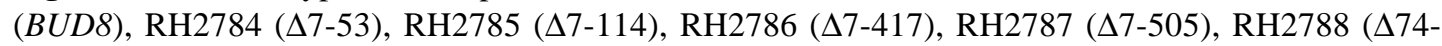

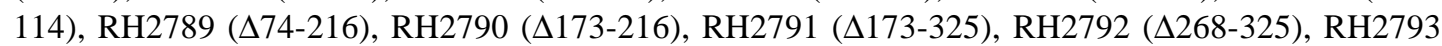
$(\Delta 268-417)$, RH2794 $(\Delta 375-417)$, RH2795 ( $\Delta 375-505)$, RH2796 ( $\Delta 468-505)$, and RH2797 $(\Delta 513-$ 600) were grown for 4 days on nitrogen starvation medium (SLAD) before pseudohyphal development of strains was visualized under a microscope and photographed. Scale bar $100 \mu \mathrm{m}$.

\section{Identification of novel Bud8p-interacting proteins by two hybrid screening}

We performed a two-hybrid screen, to isolate novel Bud8p-interacting proteins. In a first attempt, we used a hybrid protein consisting of the lexA DNA-binding domain and the full length Bud8p protein as bait. However, no positive clones were identified by expression of this fusion protein. We concluded that the two C-terminal transmembrane domains of Bud8p might interfere with nuclear localization and prevent a productive two-hybrid interaction. Therefore, a lexA-Bud8 $\mathrm{p}^{2-512}$ fusion protein was used as bait that lacks the C-terminal part carrying the two transmembrane domains. Expression of this Bud8p hybrid-protein led to the identification of three novel putative interaction partners for Bud8p (Figure 23). 
A
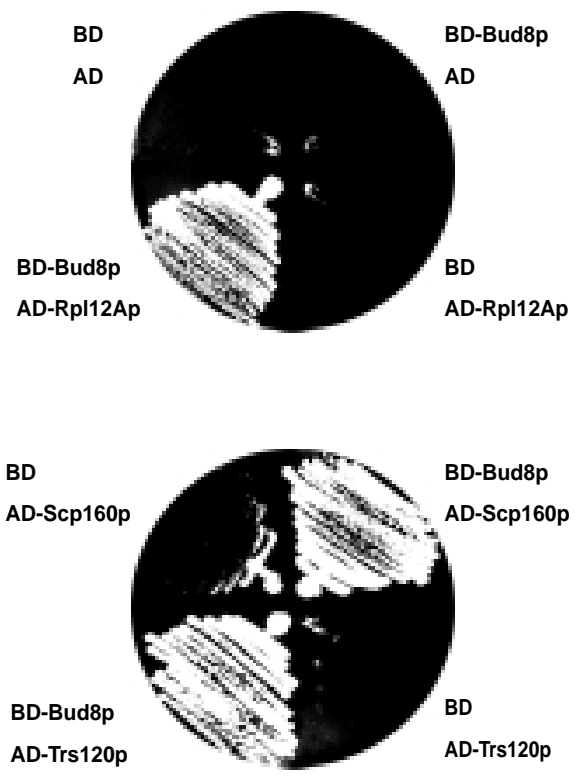

B

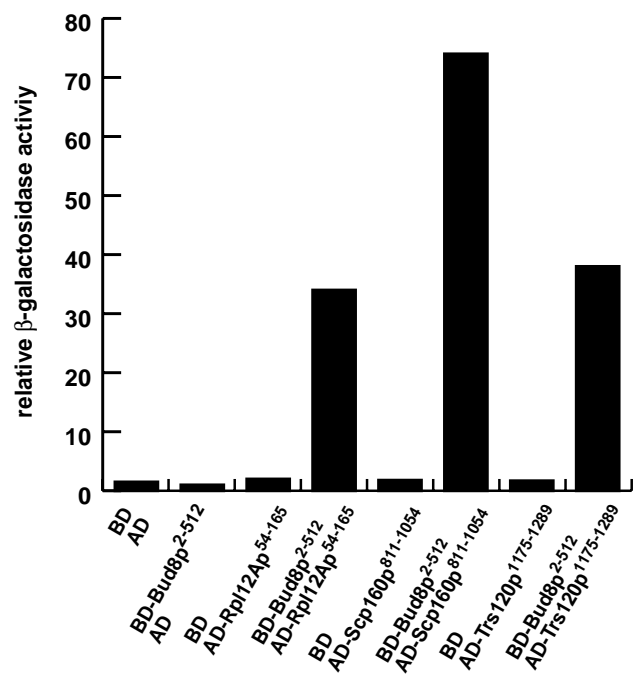

Figure 23. Two-hybrid interactions between Bud8p and Rpl12Ap, Scp160p or Trs120p. (A) Growth test for leucine (Leu) requirement. Reporter strain EGY48-p1840 was co-transformed with the indicated pairs of the following plasmids: pEG202 (BD), pJG4-5 (AD), pME2274 (BD-Bud8p), pME2271 (AD-Rp112Ap), pME2272 (AD-Scp160p), pME2273 (AD-Trs120p). Transformants were streaked onto Leu-deficient medium and incubated at $30^{\circ} \mathrm{C}$ to test for Leu-prototrophy. Plates shown were photographed after 2 days of growth. (B) Quantitative analysis of interactions. Strains described in (A) were assayed for specific B-galactosidase activity. Segments of Bud8p, Rp112Ap, Scp160p and Trs120p that are fused to either the lexA DNA-binding domain (BD) or the B42 transcriptional activation are indicated by superscript numbers. Bars and numbers represent relative activities normalized to the value obtained by the plasmid pair pJG4-5 (AD) / pME2274 (BD-Bud8p-512). All values are means of three independent measurements with a standard deviation not exceeding $20 \%$.

These proteins included Rpl12Ap, representing the 26S rRNA binding ribosomal protein L12 of S. cerevisiae (Taheri et al., 2000), Scp160p, a multiple KH-domain protein that has been found to be a component of mRNP complexes in yeast (Frey et al., 2001; Lang and Fridovich-Keil, 2000), and Trs120p, a protein that is part of the TRAPP (transport protein particle) complex, which plays a key role in the late stages of endoplasmic reticulum to Golgi traffic in the yeast (Sacher et al., 2000). In all three cases, interactions with Bud8p were found to be specific as measured by a qualitative growth assay (Figure 23A) and a quantitative reporter gene assay (Figure 23B). Sequence analysis of the plasmids isolated from the two-hybrid library revealed the identity and the exact size of the Bud8p-interacting segments of Rp112Ap, Scp160p and Trp120p. In the case of Rpl12Ap, with a length of 165 amino acids, residues 54 to 165 were found to be fused to the B42 transcriptional activation 
domain and to confer two-hybrid interaction. The part of Scp160p that was identified to interact with Bud8p encompassed residues 811 to 1054 (out of 1222), whereas the C-terminal amino acids 1175 to 1289 of Trs120p were found to confer Bud8pspecific two hybrid interaction.

Two hybrid screening performed here links Bud8p to three proteins that have been implicated in the processes of translation, mRNA transport and regulation of protein trafficking. Although interaction with these proteins was unexpected, it suggests that Bud8p might fulfill functions that are more complex than previously anticipated.

\section{Bud8p associates in vivo with Rpl12Ap and Scp160p independently of its C-terminal part}

Physical interactions between Bud8p and Rpl12Ap or Scp160p were further investigated by co-purification experiments. For this purpose, in-frame fusions between the glutathione S-transferase gene (GST) and RPL12A or GST and SCP160 were constructed and co-expressed together with a myc epitope-tagged version of BUD8. Full length versions of all proteins were used, because two hybrid measurements had involved a truncated version of Bud8p and only segments of Rp112Ap or Scp160p. Interaction between Bud8p and Trs120p could not be analyzed using this system due to instability of GST-Trs120p fusion proteins (data not shown). GST-fusion proteins were affinity-purified with glutathione beads under conditions that allow co-purification of associated proteins. Purified proteins were analyzed by Western blot analysis with either anti-GST or anti-myc antibodies (Figure 24). We found that myc-Bud8p could be very efficiently co-purified with GST-Rpl12Ap, but not with GST alone. Interestingly, the lowest molecular weight form of myc-Bud8p (running around $85 \mathrm{kD}$ ) was reproducibly enriched by co-purification with GSTRpl12Ap. When using GST-SCp160p, myc-Bud8p could also be co-purified. In contrast to GST-Rpl12Ap, however, purification was much less efficient and was completely restricted to the higher molecular portion of myc-Bud8p.

Co-purification experiments corroborated the interactions between Bud8p and Rp112Ap or Scp160p observed in the two hybrid system. Because the large Nterminal part of Bud8p was found to be sufficient for two hybrid interaction, we performed further co-purification experiments with Bud8 $\mathrm{p}^{\Delta 513-600}$, a truncated version that lacks the $\mathrm{C}$-terminal part harboring the two transmembrane domains. We found that deletion of this segment did not alter association of Bud8p with GST-Rpl12Ap or GST-Scp160p, neither quantitatively nor qualitatively (Figure 24A). 
Thus, association of Bud8p with Rpl12Ap or Scp160p does not require the Cterminal residues 513 to 600 .

A

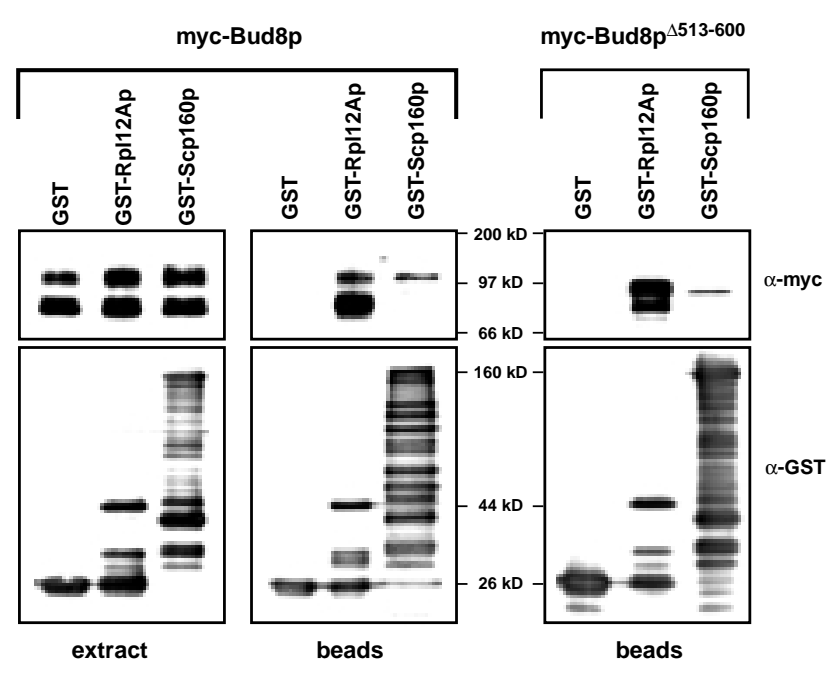

B

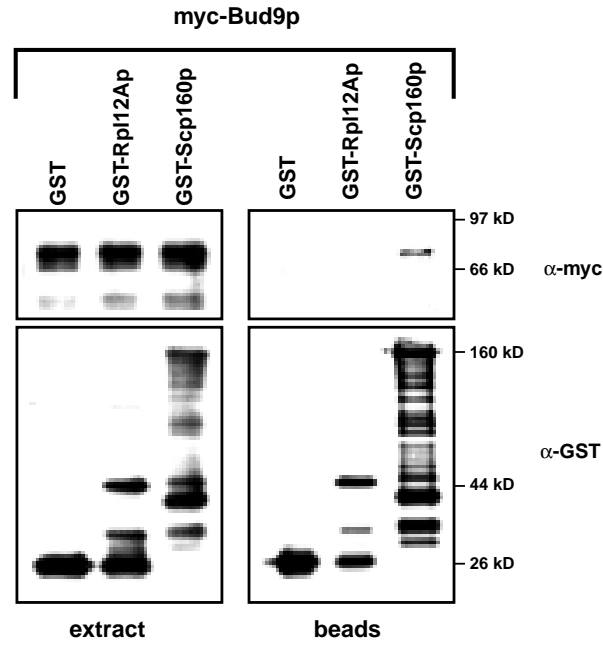

Figure 24. Interactions between Bud8p, Bud9p, Rpl12Ap, Scp160p and Trs120p proteins. (A) Copurification of myc-Bud8p and myc-Bud8 $\mathrm{p}^{\Delta 513-600}$ proteins with either GST-Rpl12Ap, GST-Scp160p or GST-Trs120p. Total protein extracts were prepared from strain RH2495 carrying either of the plasmid pairs pME2319 (myc-Bud8p) and pYGEX-2T (GST), pME2319 and pME2247 (GSTRp112Ap), pME2319 and pME2248 (GST-Scp160p), pME2319 and pME2336 (GST-Trs120p), pME2298 (myc-Bud8p ${ }^{\Delta 513-600}$ ) and pYGEX-2T, pME2298 and pME2247, pME2298 and pME2248 or pME2298 and pME2336. GST and GST fusion proteins were purified as described. Equivalent amounts of each extract obtained before (extract) or after (beads) GST-affinity purification was subjected to SDS-page, transferred to nitrocellulose and probed with an anti-GST antibody ( $\alpha$-GST) or an anti-myc antibody $(\alpha$-myc) for detection of GST, GST fusion proteins and associated myc tagged proteins. Molecular size standards (in $\mathrm{kDa}$ ) are shown as reference. (B) Co-purification of myc-Bud9p with GST-Rpl12Ap or GST-Scp160p. Protein extracts were prepared from strain RH2495 carrying plasmid pME1939 (myc-Bud9p) together with either pYGEX-2T, pME2247, pME2248 or pME2336 and analyzed for protein-protein interactions as described in (A).

\section{Bud9p associates with Scp160p, but not with Rpl12Ap}

Our previous studies have shown that Bud8p associates with Bud9p in vivo (Taheri et al., 2000). In addition, Bud9p has been found to partially fulfill the function(s) of Bud8p, when expressed from the $B U D 8$ promoter, suggesting overlapping function(s) of Bud8p and Bud9p (Schenkman et al., 2002). Therefore, we tested whether Bud9p might also associate with Rp112Ap or Scp160p. Co-purification experiments were performed exactly as described above, exception that a myc epitope-tagged version of $B U D 9$ was expressed instead of $B U D 8$ together with either GST-RPL12A or GST-SCP160 fusions (Figure 24B). In contrast to myc-Bud8p, mycBud9p could not be co-purified in detectable amounts together with GST-Rpl12Ap. When using GST-Scp160p, however, myc-Bud9p could be co-purified to amounts 
comparable to myc-Bud8p. Thus, in vivo association with Rpl12Ap appears to be specific for Bud8p, whereas Scp160p is able to interact with both Bud8p and Bud9p.

\section{Deletion of the transmembrane helices restricts localization of Bud8p to subcellular regions enriched for Rpl12Ap and Scp160p}

The fact that interaction of Bud8p with Rpl12Ap and Scp160p is executed by the large N-terminal part of Bud8p is surprising, because this segment is predicted to reside in the extracytoplasmic space (Ni and Snyder, 2001), whereas Rpl12Ap and Scp160p have been described to reside in the cytoplasm (Briones et al., 1998; Frey et al., 2001; Lang and Fridovich-Keil, 2000). Because previous studies had used haploid strains for localization studies, we determined subcellular localization of Rpl12Ap and Scp160p in diploid cells. Homozygous diploid strains were constructed, that expressed myc epitope-tagged versions of either RPL12A or $S C P 160$ instead of the endogenous RPL12A or SCP160 genes, respectively, or that expressed GFP-RPL12A or GFP-SCP160 fusion genes at endogenous levels. In both cases, Rpl12Ap and Scp160p, myc-tagged proteins and GFP-fusions were found to produce at predominantly cytoplasmic staining (Figure 25). In addition, myc epitope tagged version appeared to be enriched around the nuclei, indicating ER association (Figure 25A), whereas GFP-fusions indicated vacuolar exclusion of both proteins (Figure 25B).

The C-terminal segment of Bud8p (residues 513 - 600) carries two transmembrane domains that have been suggested to be required for membrane association (Harkins et al., 2001; Taheri et al., 2000). This segment of Bud8p is functionally interchangeable with the corresponding part of Bud9p, which shares $52 \%$ similarity with Bud8p in this region and also contains two transmembrane domains (Schenkman et al., 2002). We found that residues 513 - 600 of Bud8p are required for functionality, but not for interaction with Rp112Ap or Scp160p (compare Bud8 $\mathrm{p}^{\Delta 513-600}$ in Figures 20 and 24). To further characterize the function of this part of Bud8p, we tested whether it might be required for distal pole localization by determining the subcellular localization of Bud8 $\mathrm{p}^{\Delta 513-600}$, a truncated version that lacks the C-terminal region. We found that $\mathrm{Bud} 8 \mathrm{p}^{\Delta 513-600}$ is predominantly distributed throughout the cell and is no longer concentrated at the distal pole, when compared to full length Bud8p (Figure 26). Remarkably, localization of Bud8 $\mathrm{p}^{\Delta 513-600}$ was found to be very similar to that detected for Rpl12Ap and Scp160p (compare Figure 25A and 26). 
A

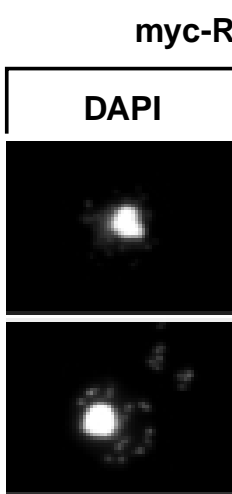

myc-Rpl12Ap

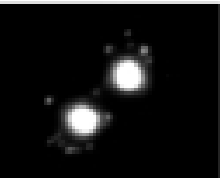

\section{FITC}
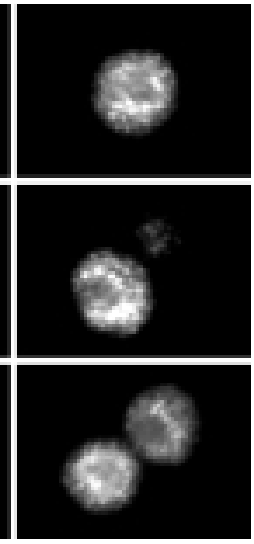

B
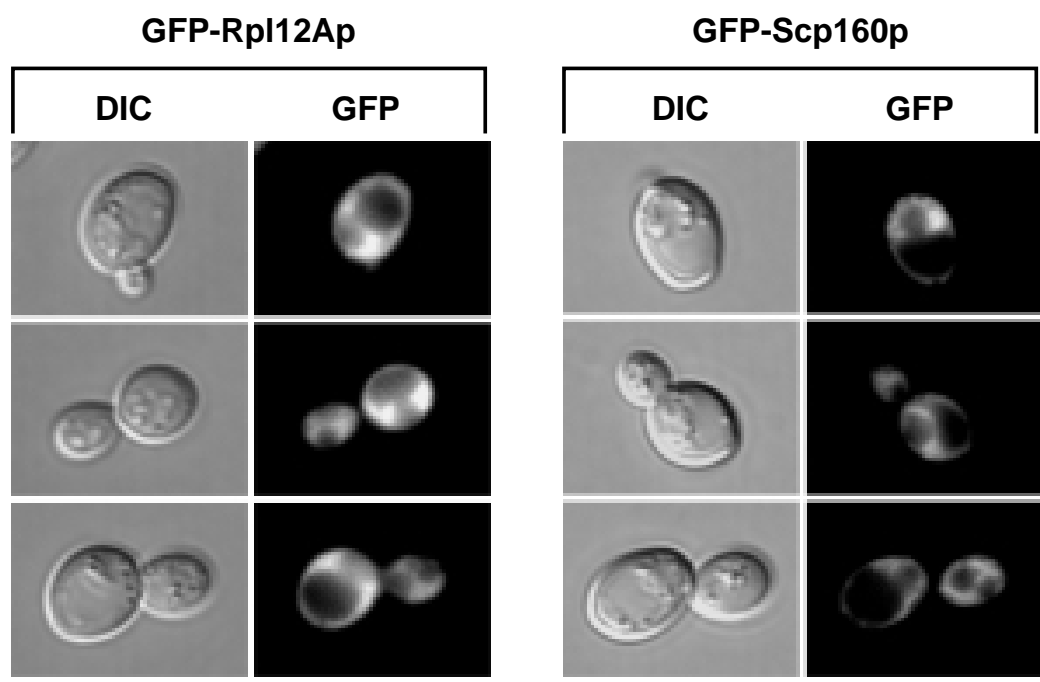

Figure 25. Subcellular localization of Rpl12Ap and Scp160p. (A) Immunofluorescence microscopy. Yeast strains expressing endogenous levels of myc-Rpl12Ap (RH2745) or myc-Scp160p (RH2748), respectively, were grown to exponential phase and prepared for anti-myc immunofluorescence microscopy. Representative cells of each strain are shown that were viewed for DNA with DAPI imaging (DAPI) or for anti-myc immunofluorescence (FITC). Scale bar applies to (A) and (B) and equals $5 \mu \mathrm{m}$. (B) Living cells of yeast strains expressing GFP-Rpl12Ap (RH2746) or GFP-Scp160p (RH2749) at endogenous levels were viewed by differential interference contrast microscopy (DIC) and by fluorescence microscopy (GFP) after growth to exponential phase.

Thus, deletion of the transmembrane domains not only suppresses polar localization of Bud8p, but also restricts the protein to subcellular regions that are enriched for Rp112Ap and Scp160p. These findings further support functional interactions between the N-terminal part of Bud8p and Rp112Ap or Scp160p in vivo. 


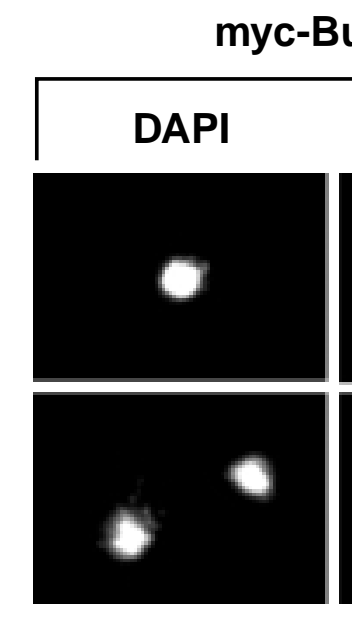

myc-Bud8p

\section{FITC}
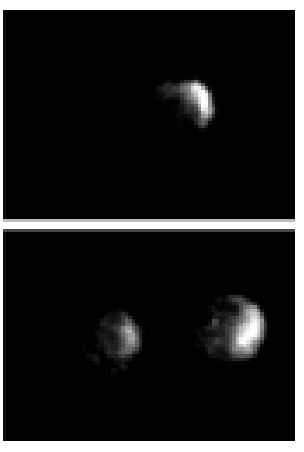

myc-Bud8p ${ }^{\Delta 513-600}$

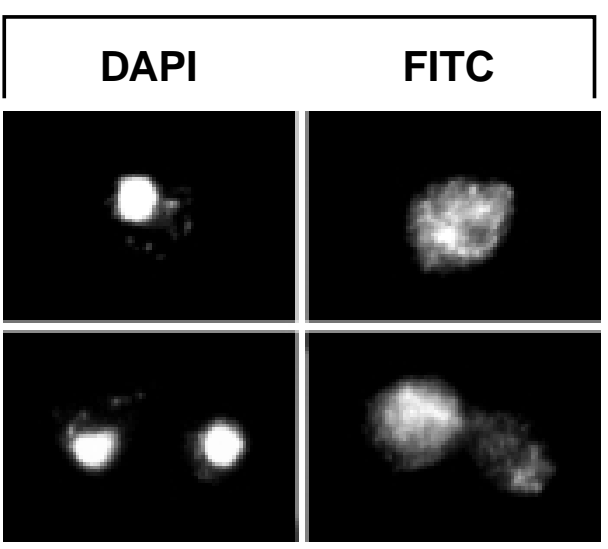

Figure 26. Requirement of the C-terminal transmembrane domain for polar localization of Bud8p. Strain RH2449 (bud8Abud84) expressing myc-Bud8p (pME2318) or myc-Bud8p $\mathrm{p}^{\Delta 513-600}$ (pME2297) was grown to exponential phase and prepared for anti-myc immunofluorescence microscopy. Representative cells of each strain are shown that were viewed for DNA with DAPI imaging (DAPI) or for anti-myc immunofluorescence (FITC). Scale bar $5 \mu \mathrm{m}$.

\section{RPL12A and SCP160 are required for regular bud site seletion, but not for polar localization of Bud8p}

The finding that Bud8p interacts with Rpl12Ap, Scp160p and Trs120p suggested a possible involvement of these proteins in regulation of cell polarity. A previous study had shown that diploid strains lacking $R P L 12 B$, representing a duplicated isogene of $R P L 12 A$, produce more than $50 \%$ cell with a random budding pattern ( $\mathrm{Ni}$ and Snyder, 2001). A role of SCP160 or TRS120 in bud site selection has not yet been reported. Here, we measured the effects of deleting either RPL12A or SCP160 on bud site selection. As a control, we constructed diploid strains lacking RPL12B. TRS120 was not amenable to a deletion analysis, because this gene is essential for cell viability. We found that both diploid $r p l 12 A \Delta / r p l 12 A \Delta$ and scp 160 $\Delta / s c p 160 \Delta$ mutant strains had lost the typical bipolar budding pattern and produced significantly higher amounts of cells with a random budding pattern, as found for the $r p l 12 B \Delta / r p l 12 B \Delta$ mutant. In case of $r p l 12 A \Delta r p l 12 A \Delta$ and $r p l 12 B \Delta / r p l 12 B \Delta$ strains, the percentage of cells with random bud sites was increased more than 10-fold. In the

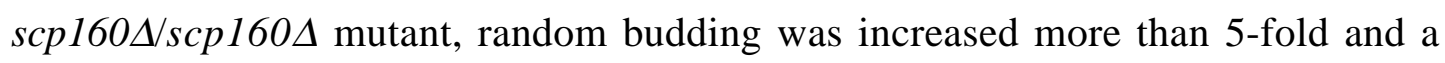
larger proportion of unipolar budding cells were found (Figure 27). 
A

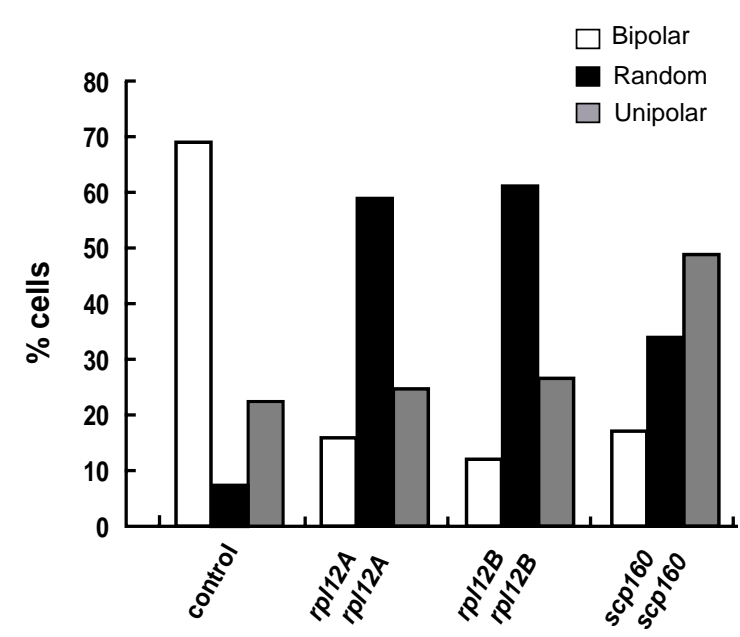

B

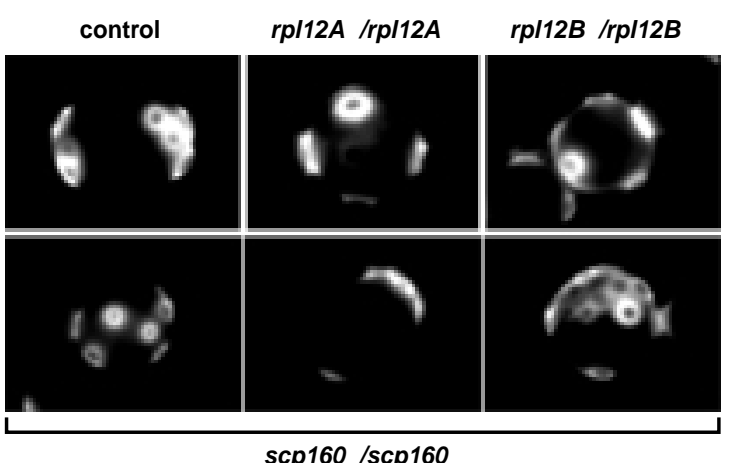

C

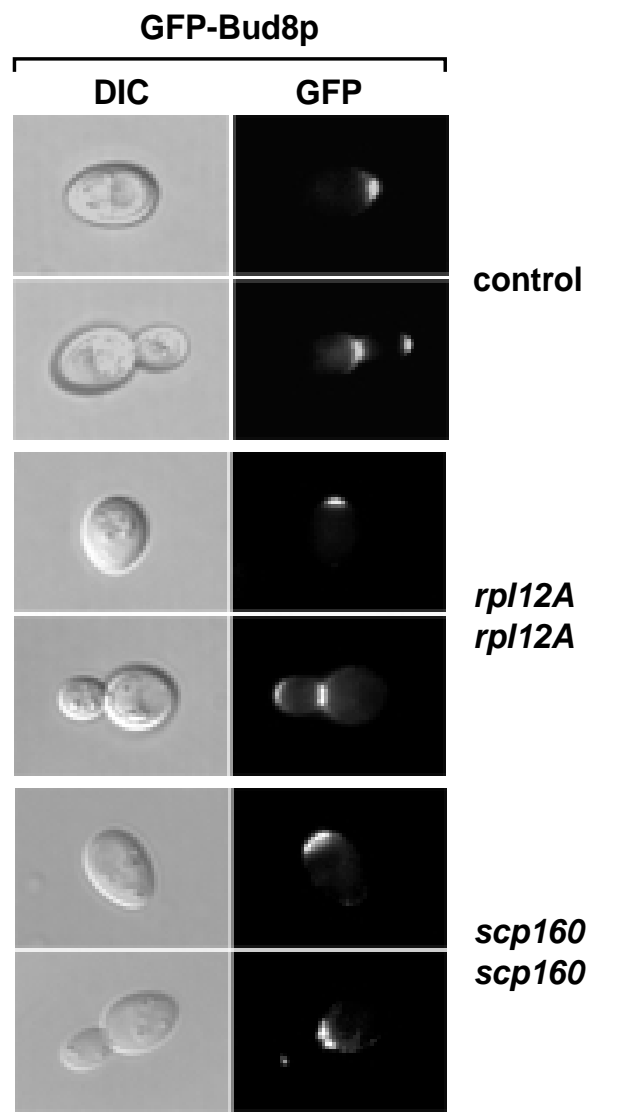

Figure 27. Regulation of bud site selection by RPL12A and SCP160. (A) Quantitative analysis of bud scar distribution. Strains RH2782 (control), RH2779 (rpl12A 4 rpl12A $\Delta$ ) and RH2780

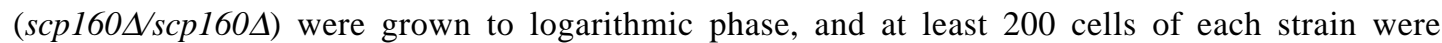
analyzed for bud scar distribution. Bars represent the percentage of cells exhibiting a bipolar (white bars), random (black bars) or unipolar (gray bars) budding patterns. (B) Fluorescence imaging of representative cells of strains described in (A). Scale bar $5 \mu \mathrm{m}$. (C) Localization of GFP-Bud8p in the absence of Rpl12Ap or Scp160p. Living cells of yeast strains Strains RH2782 (control), RH2779 (rpl12ALrpl12A4) and RH2780 (scp1604scp1604) expressing GFP-Bud8p (pME1772) were viewed by differential interference contrast microscopy (DIC) and by fluorescence microscopy (GFP) after growth to exponential phase.

Thus, Rpl12Ap, Rpl12Bp and Scp160p are required for proper bud site selection and cell polarity. A GFP-Bud8p fusion protein was expressed and visualized in diploid $r p l 12 A \Delta / r p l 12 A \Delta$ and $s c p 160 \Delta / s c p 160 \Delta$ mutant strains, to determine the requirement of Rpl12Ap and Scp160p for distal localization of Bud8p. We found that deletion or neither RPL12A nor SCP160 altered the intracellular localization of GFP-Bud8p at the distal cell pole (Figure 27C). 
Thus, neither Rpl12Ap nor Scp160p appear to control cell polarity by affecting polar localization of Bud8p. Because these proteins are required for proper bud site selection and have been identified to interact with Bud8p, they might fulfill a function in the cell polarity establishment pathway that is located downstream of Bud8p.

\section{Discussion}

Yeast cells must choose a site for bud initiation once during every round of cell division. This selection process is under control of landmark proteins that are thought to tag the site for cell division. In order to investigate the molecular function of landmark proteins, we performed a detailed structural and functional analysis of Bud8p, a transmembrane glycoprotein that is thought to tag the distal pole of yeast cells. Our study not only identifies several functional domains within Bud8p, but also provides evidence for a novel mechanism, by which spatial landmark proteins might control cell polarity and directed cell division. Based on our data, we suggest that Bud8p helps to establish cell polarity by physical interaction with the ribosomal protein Rpl12Ap (and Rpl12Bp) and with Scp160p and Trs120p, factors that are associated with the endoplasmic reticulum and the Golgi network. Interaction of Bud8p with these factors is established by the large N-terminal part, whereas the Cterminal transmembrane domain is required to localize Bud8p (and possibly associated factors) to the distal cell pole. This model is supported by the physical interactions between Bud8p, Rpl12Ap and Scp160p that were demonstrated by two independent methods, the two-hybrid system and co-purification experiments. Functional relevance of these interactions is supported by the fact that all three proteins are required for bipolar budding, a process that involves a group of not more than 130 proteins representing only $2.1 \%$ of all yeast proteins ( $\mathrm{Ni}$ and Snyder, 2001). Further support comes from the finding that subcellular localization of Rpl12Ap and Scp160p are very similar and overlap with a version of Bud8p $\left(B u d 8 p^{\Delta 513-600}\right.$ ) that is sufficient for physical interaction with Rpl12Ap and Scp160p. Finally, the large N-terminal portion of Bud8p not only interacts with Rpl12Ap and Scp160p, but also harbors several functionally distinct domains, further emphasizing the functional relevance of the physical interactions between these proteins.

At first sight, our findings appear to be in contrast with the previously proposed model of Bud8p topology and function. 
Based on genetic evidence and in analogy to the haploid-specific landmark protein Bud10p/Ax12p, previous studies have suggested that Bud8p might recruit the Rsr1p/Bud2p/Bud5p GTPase module via interaction with the small cytoplasmic region located between the two transmembrane domains at the $\mathrm{C}$-terminus. The large $\mathrm{N}$-terminal part of Bud8p was predicted to reside in the extracellular space, owing the fact that it contains the sites for $\mathrm{N}$ - and O-linked glycosylation of Bud8p (Harkins et al., 2001). In view of this topology of Bud8p, we were surprised to find a series of cytoplasmic proteins that specifically interact with the $\mathrm{N}$-terminal portion of Bud8p. A possible explanation migt be that Bud8p interacts with cytoplasmic proteins as such Rp112Ap, Scp160p and Trs120p into ER. A further and more speculative possiblity is that after the transport of Bud8p to the distal pole its looking might be inverted with the $\mathrm{N}$-terminus locating into the cytoplasm (which appears to involve the transmembrane domain). Bud8p might help to recruit a subset of ribosomes and part of the ER and Golgi compartment to the distal pole. In turn, these subcellular structures might allow synthesis of proteins required for initiation of cell division at or close to the distal cell pole. The large number of RNA binding domains in Scp160p (14 KH domains) provides the contacts with mRNAs and rRNAs, allowing selective binding to polysosomes. The association of Scp160p with membrane-bound polysomes suggest that the protein may play important roles in positioning specific mRNAs at the ER and regulating their translation at this site (Frey et al., 2001; Lang and Fridovich-Keil, 2000; Lang et al., 2001). Therefore, Scp160p might be a component of the general translation machinery involved in the translation of various mRNA including ASH1. In S. cerevisiae, Ash1p is a specific repressor of transcription that localizes asymmetrically to the daughter cell nucleus through the localization of ASH1 mRNA to the distal tip of the daughter cell (Bobola et al., 1996). Recently, it has been shown that Scp160p is probably indirect required for ASH1 mRNA localization, because ASH1 mRNA is partially delocalized in scp160 mutant strain (Irie et al., 2002). However, ASH1 is not required for proper bipolar bud site selection, suggesting that Bud8p, Rpl12Ap and Scp160p may be involved in transport of other mRNAs to the distal cell pole, e. g. of factors that regulate budding.

The interaction of Bud8p with Trs120p suggest that the targeting of Bud8p to the bud tip is possible by participate of components in vesicle transport pathway. Trs120p is a subunit of TRAPP complex that associates stabls with the cis-Golgi complex and mediates the targeting of ER-to-Golgi vesicles to the Golgi apparatus (Barrowman et al., 2000; Wang et al., 2000). TRAPP subunits can activate as GEF (guanine 
nucleotide exchange factor) the Ypt31p GTPase that is required for ER-to-Golgi transport (Jones et al., 1993). Ypt31p function is essential for protein transport from the trans-Golgi. A genomic study has shown that Ypt31p and other two vesicle transport proteins (Vam3p and Vam8p) are specific for bipolar budding pattern. ypt31 $\Delta y p t 31 \Delta$, and other mutant strains display random bud site selection pattern and exhibit defects in Bud8p-GFP localization (Ni and Snyder, 2001). It is possible, that Trs120p with other proteins in vesicle transport, are involved in the targeting of Bud8p protein to the distal bud site.

Our study further suggests that Bud8p (and Bud9p) must harbor at least two distinct types of domains, one required for proper localization and a second for recruitment of downstream components for bud initiaion to the respective cell pole. At first sight the localization domains of Bud8p and Bud9p should be different as they one functions of different cell poles. The second type of domains should be similar as both proteins might compete for the same factors of the budding machinery. That would predict that $\mathrm{N}$-terminus is important for function because highest similarity (44\%). Vice versa, N-terminal part should harbor localization information, because there is no real overlap. Such a model is supported by promoter swap experiments and studies employing chimeras (Harkins et al., 2001; Schenkman et al., 2002). However, our study suggests that the structure of Bud8p is more complicated and that regulation and function of Bud8p might be much more complex than previously anticipated. Our deletion analysis has identified at least three functionally distinct domains within the $\mathrm{N}$-terminal part of the protein, namely (i) residues 2 to 53 that negatively act on distal budding, (ii) residues 74 to 173 and 417 to 468 that are essential for distal bud site selection, and (iii) residues 216 to 373 that when absent prevent recognition of the cell poles at all. Some of these functions must be Bud8pspecific, because exchange of the N-terminal part for Bud9p does not allow rescue of bud8 deletion, whereas a BUD8N-Bud9C chimera is able to do so (Schenkman et al., 2002). Indeed, we here find that the N-terminal part of Bud8p interact specifically with Rpl12Ap, whereas we do not find any interaction between Rpl12Ap and Bud9p. It will be interesting to see which part of the protein confers this interaction. On the other hand, Scp160p interacts with both proteins. It is possible that Bud9p regulate the cell polarity and directed cell division by other way that is not similar to Bud8p. Therefore, Bud9p does not need probably the function of ribosomal proteins for the regulation of cell polarity. Bud9p may interact indirectly with Scp160p by involving other protein that is necessary for Bud8p-regulation pathway. 


\section{Materials and methods}

\section{Yeast strains and growth conditions}

All yeast strains used in this study are congenic to the $\Sigma 1278 \mathrm{~b}$ genetic background with the exception of strain EGY48-p1840 that was used for two-hybrid analysis (Table VII). rpl12A $4:: k a n R$ and scp1604::kanR deletion mutations were introduced using plasmids pM2302 and pME2303 (Table VIII). Strains RH2783 - RH2792 all expressing $m y c$ epitope-tagged versions of $B U D 8$ at endogenous levels, were obtained by integration of linearized plasmids pME2321 - pME2335. Strains RH2745, RH2746, RH2748 and RH2749, which all express either myc epitopetagged versions or green fluorescence protein (GFP) fusions of RPL12A or SCP160 at endogenous levels, were obtained by integration of linearized plasmids pME2252, pME2253, pME2254 or pME2255. Standard methods for genetic crosses and transformation were used and standard yeast culture YPD, YNB and SC media were prepared essentially as described (Guthrie and Fink, 1991). Low ammonium medium (SLAD) was prepared as described (Gimeno et al., 1992). Solid SLAD 2\% agar media was used for qualitative pseudohyphal growth assays.

\section{Pseudohyphal growth assays}

Assays for pseudohyphal development were performed as described previously (Mösch and Fink, 1997). After 3 days of growth on solid SLAD medium, pseudohyphal colonies were viewed with a Zeiss Axiolab microscope and photographed using a digital camera DX30 and the Kappa Image Base Noah software (Kappa Opto-Electronics, Gleichen, Germany).

\section{Plasmid Constructions}

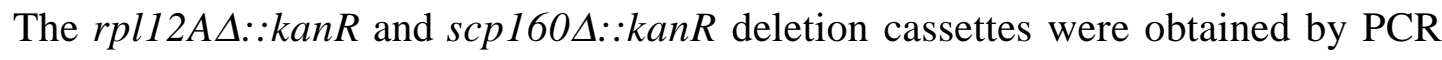
amplification using genomic DNA of corresponding strains from the EUROSCARF project (Winzeler et al., 1999). Amplified fragments were inserted into vector pBluescriptKS (Stratagene, ) to obtain plasmids pME2302 and pME2303. Plasmid pME2320 was obtained by subcloning of a $4.0 \mathrm{~kb}$ genomic BUD 8 fragment from pME1769 (Taheri et al., 2000) into pRS306 (Sikorski and Hieter, 1989) using restriction enzymes $\mathrm{XhoI}$ and $\mathrm{BamHI}$. Plasmid pME2274 was constructed by PCR amplification of a $1.5 \mathrm{~kb}$ XhoI-XhoI BUD8 fragment coding for Bud8p amino acid residues 2 to 512, followed by insertion of this fragment into the XhoI site of plasmid pEG202 (Fashena et al., 2000). Plasmids pME2318, pME2319 and pME2321, all expressing a $m y c^{6}$ epitope-tagged version of $B U D 8$ under control of the $B U D 8$ 
promoter, were obtained by insertion of two tandemly repeated 120 bp BamHI fragments carrying the triple myc epitope $\left(m y c^{3}\right)$ after the start codon of $B U D 8$. The set of $m y c^{6}-B U D 8$ alleles carrying systematic deletions in the BUD8 open reading frame was contructed by a PCR-based strategy involving several steps. (i) A set of six BUD8 fragments was amplified by PCR using plasmid pME2321 as template and introducing unique NotI and $B g l \mathrm{II}$ restriction sites. Amplified fragments consisted of the $B U D 8$ promoter region, the $B U D 8$ start codon followed by the $m y c^{6}$ epitope tag and segments of the $B U D 8$ open reading frame starting at the second codon and ending at either of the codons 7, 74, 173, 268, 375 or 468 (Figure 20). (ii) A second set of $B U D 8$ fragments was amplified by PCR introducing unique $B g l \mathrm{II}$ and $\mathrm{XhoI}$ sites and consisting of $B U D 8$ coding region starting at codons 53, 114, 216, 325, 417 or 505 and ending at the stop codon, followed by the BUD8 terminator region. (iii) Fragments from (i) and (ii) were individually cloned by insertion into plasmid pME2256 by using restriction enzymes Not I and $B g l \mathrm{II}$ or $B g l \mathrm{II}$ and $X h o \mathrm{I}$, respectively. (iv) The $m y c^{6}-B U D 8$ alleles carrying the desired deletions were constructed by combination of the respective fragments from (iii) and were finally subcloned into the integrative vector pRS306 to obtain plasmids pME2322 pME2334. Plasmid pME2297 was obtained by two steps. (i) The SalI-XhoI fragment of pME2318 was replaced for the SalI-XhoI fragment from pME2274 (lacking the Cterminal part of $B U D 8$ ). (ii) A XhoI fragment was inserted into the XhoI site of the construct obtained in (i), which was amplified by PCR and consisted of BUD8 codons 601 to 603 , the stop codon and the $B U D 8$ terminator region. Plasmids pME2298 and pME2335 were obtained by subcloning of a $3.3 \mathrm{~kb}$ ApaI-NotI fragment from pME2297 into vectors pRS425 or pRS306, respectively. Plasmids pME2254 and pME2255, expressing either GFP-RPL12A from the RPL12A promoter or GFP-SCP160 from the $S C P 160$ promoter, were constructed by introducing a $B g l \mathrm{II}$ site in front of the second codon of RPL12A or SCP160 and insertion of a $750 \mathrm{bp} B g l \mathrm{II}$ fragment encoding the GFPuv variant of GFP (Clontech, Heidelberg, Germany). Plasmids pME2252 and pME2253, expressing myc epitopetagged versions of either RPL12A under control of the RPL12A promoter or SCP160 from the SCP160 promoter, were obtained by insertion of a single $\left(m y c^{3}-R P L 12 A\right)$ or three tandemly repeated copies $\left(m y c^{9}-S C P 160\right)$ of a 120 bp BamHI fragment carrying the triple myc epitope $\left(\mathrm{myc}^{3}\right)$ after the start codon of RPL12A or SCP160. Plasmids pME2247, pME2248 and pME2336 were obtained by N-terminal fusion of RPL12A, $S C P 160$ or TRS120 open reading frames to GST in vector pYGEX-2T (Schlenstedt et al., 1995). 


\section{Two-hybrid screen}

Two-hybrid analysis tools: plasmids pEG202 and pJG4-5, a yeast genomic library cloned in vector pJG4-5 and the yeast strain EGY48-p1840 were kindly provided by Erica Golemis and Roger Brent (Fashena et al., 2000; Gyuris et al., 1993). For the selection of Bud8p-interaction partners by the two-hybrid system, yeast strain EGY48-p1840 containing $B U D 8^{2-512}$ as a bait on plasmid pME2274 was transformed with a yeast genomic interaction library cloned into pJG4-5 by the lithium acetate method (Ito et al., 1983) to obtain $5 \times 10^{6}$ transformants. Transformants were collected as a pool, and Leu-prototrophic strains were selected by growth on SC medium lacking Leu, Trp and His, and containing 2\% galactose and $1 \%$ raffinose, and assayed for $\beta$-galactosidase activiy as described by Fashena and Golemis (Fashena et al., 2000). Library plasmids were isolated as described (Hoffman and Winston, 1987) and analyzed by DNA sequencing using the ABI Prism Big Dye terminator sequencing kit and an ABI 310 Genetic Analyzer (Applied Biosystems, Weiterstadt, Germany). Interactions were verified by reintroducing library plasmids into the parental strain EGY48-p1840 carrying either pME2274 or pEG202, followed by a growth test for Leu-prototophy.

Two-hybrid interactions were quantified by measuring specific $B$-galactosidase activities of strain EGY48-p1840 that was co-transformed pairwise with the various pEG202 and pJG4-5 based plasmids. Transformants were grown in liquid SC - His Trp medium to an $\mathrm{OD}_{600}$ of 0.5 to 1.0 , and protein extracts were prepared and assayed for specific B-galactosidase activities as previously described (Rose and Botstein, 1983). Specific ß-galactosidase activities equal $\left(\mathrm{OD}_{420} \times 1.7\right) /(0.0045 \times$ protein concentration $\mathrm{x}$ extract volume $\mathrm{x}$ time). Assays were performed on at least three independent transformants, and standard deviation did not exceed $20 \%$.

\section{Analysis of budding patterns}

Budding patterns were determined by bud scar staining and time lapse-microscopy essentially as previously described (Kron et al., 1994; Pringle, 1991; Taheri et al., 2000). For bud scar staining, yeast cells were grown in liquid YPD medium at $30^{\circ} \mathrm{C}$ to an $\mathrm{OD}_{600}$ of 0.6 , and fixed at room temperature for $2 \mathrm{~h}$ in $3.7 \%$ formaldehyde. Samples were rinsed twice in water and resuspended in $200 \mu \mathrm{l}$ of a fresh stock of 1 $\mathrm{mg} / \mathrm{ml}$ calcofluor white (Fluorescent Brightener F-6259, Sigma). Bud scars were visualized by fluorescence microscopy using a Zeiss Axiovert microscope and photographed using a Hamamatsu Orca ER digital camera and the Improvision Openlab software (Improvision, Coventry, England). Cells with two to 10 obvious bud scars were divided into three classes: bipolar, cells with two or more bud scars 
with at least one scar at each end of the cell (the birth end and the free end); unipolar, cells with all bud scars at one end of the cell immediately adjacent to one another; random, cells with bud scar distributions other than bipolar or unipolar. Numbers in the tables represent the percentage of cells in each class for a sample of at least 200 cells. Time lapse microscopy was performed by using a chamber for high magnification imaging of yeast growth as described previously (Kron et al., 1994). Positions of bud site emergence were determined by direct microscopic observation. For each strain measured, at least 70 cell divisions were observed.

\section{GFP fluorescence and indirect immunofluorescence microscopy}

Yeast strains expressing GFP fusion proteins were grown to exponential phase in liquid YNB medium as describe for bud scar staining. Cells from $1 \mathrm{ml}$ of the cultures were harvested by centrifugation and immediately viewed in vivo by either differential interference contrast microscopy (DIC) or fluorescence microscopy using a GFP filter set (AHF Analysentechnik AG, Tübingen, Germany). Cells were photographed using a Hamamatsu Orca ER digital camera and the Improvision Openlab software (Improvision, Coventry, UK). For immunofluorescence microscopy, cells were cultured as for GFP microscopy, fixed in 3.7\% formaldehyde and spheroblasts were prepared as described (Pringle et al., 1991). 4',6-diamidino-2phenylindole (DAPI) staining and monoclonal mouse anti-myc antibodies (9E10) together with an Alexa 488-conjugated goat anti-mouse antibody (Molecular Probes, OR, USA) were used for visualization of nuclei and myc epitope-tagged proteins, respectively. Cells were viewed and photographed as described above using standard DAPI and fluorescein isothiocyanate (FITC) filter sets.

\section{Protein analysis and co-purification experiments}

Extracts were prepared from cultures grown to exponential phase in YNB medium. Briefly, cultures were washed in ice-cold buffer $\mathrm{R}(50 \mathrm{mM}$ Tris- $\mathrm{HCl} \mathrm{pH} 7.5,1 \mathrm{mM}$ EDTA, $50 \mathrm{mM}$ dithiothreitol), lysed with glass beads in $200 \mu \mathrm{l}$ of buffer R + PIM (1 $\mathrm{mM}$ each phenylmethylsulfonyl fluoride, TLCK, TPCK, $p$-aminobezamidine-HCl and $o$-phenanthroline) $+3 \%$ Triton $\mathrm{X}-100+1 \%$ SDS at $4{ }^{\circ} \mathrm{C}$, and spun at 3000 r.p.m. for $5 \mathrm{~min}$ to remove glass beads and large cell debris. Extracts $(10 \mu \mathrm{l})$ were removed to determine total protein concentration using a protein assay kit (Bio-Rad, München, Germany). SDS loading dye was added to the remaining total extracts and proteins were denatured by heating at $65^{\circ} \mathrm{C}$ for $10 \mathrm{~min}$. Equal amounts of proteins were subjected to SDS-PAGE and transferred to nitrocellulose membranes. Myc epitopetagged proteins were detected using ECL technology (Amersham, UK) after 
incubation of membranes with a monoclonal mouse anti-myc antibody (9E10) and a peroxidase-coupled goat anti-mouse secondary antibody (Dianova, Hamburg, Germany).

For purification of GST fusions and associated proteins, extracts of strains coexpressing GST fusion proteins together with myc-tagged versions of Bud8p or Bud9p were prepared after growth on galactose medium for $6 \mathrm{~h}$ exactly as previously described (Roberts et al., 1997). Extracts were incubated with glutathione-agarose overnight at $4{ }^{\circ} \mathrm{C}$ in the presence of the complete protease inhibitor cocktail at twice the working concentration according to the manufacturers guidelines (Roche Diagnostics GmbH, Mannheim, Germany). Beads were repeatedly washed and collected to purify GST fusions and any associated proteins. Samples were denatured by heating at $60^{\circ} \mathrm{C}$ for $5 \mathrm{~min}$ in SDS sample buffer and equal amounts of each samples were analysed by Western blot as described above using either polyclonal anti-GST antibody (Santa Cruz Biotechnologies, CA) or the monoclonal mouse anitmyc antibody (9E10).

Table VII. Strains used in this study

\begin{tabular}{|c|c|c|}
\hline Strain & Genotype & Source \\
\hline EGY48-p1840 & $\begin{array}{l}\text { MAT } \alpha, \text { trp1, his3, 6xlexAops-LEU2, } \\
\text { 1xlexAop-lacZ, GAL1-lacZ-URA3 }\end{array}$ & (Fashena et al., 2000) \\
\hline RH2495 & $\begin{array}{l}\text { MATa/MAT } \alpha, \text { ura3-52/ura3-52, } \\
\text { leu2::hisG/leu2::hisG, his3::hisG/HIS3, } \\
\text { trp1::hisG/TRP1 }\end{array}$ & (Taheri et al., 2000) \\
\hline RH2745 & 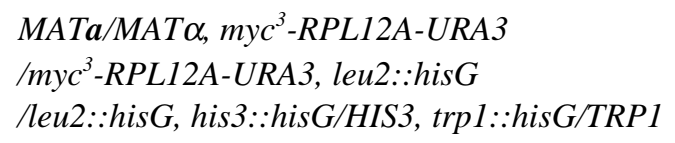 & this study \\
\hline RH2746 & 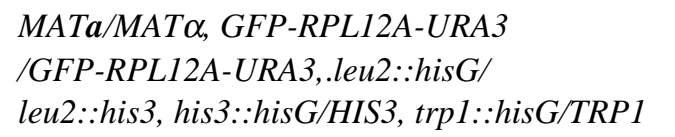 & this study \\
\hline RH2748 & $\begin{array}{l}\text { MATa/MAT } \alpha, \text { myc }{ }^{9}-S C P 160-U R A 3 \\
\text { myc }{ }^{9}-S C P 160-U R A 3, \text { leu2::hisG } \\
\text { /leu2::hisG, his3::hisG/HIS3, trp1::hisG/TRP1 }\end{array}$ & this study \\
\hline RH2749 & $\begin{array}{l}\text { MATa/MAT } \alpha, \text { GFP-SCP160-URA3 } \\
\text { GFP-SCP160-URA3, leu2::hisG } \\
\text { /leu2::hisG, his3::hisG/HIS3, trp1::hisG/TRP1 }\end{array}$ & this study \\
\hline RH2779 & 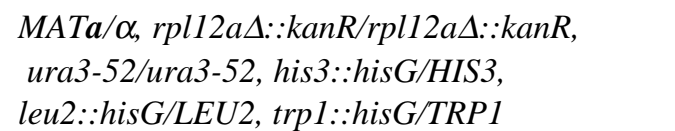 & this study \\
\hline
\end{tabular}


Table VII. Strains used in this study

\begin{tabular}{|c|c|c|}
\hline Strain & Genotype & Source \\
\hline RH2780 & 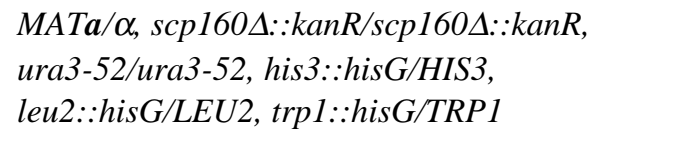 & this study \\
\hline RH2781 & 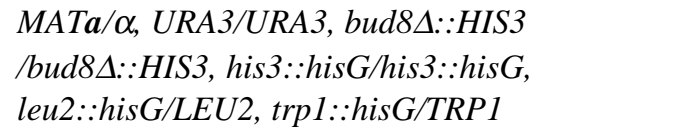 & this study \\
\hline RH2782 & 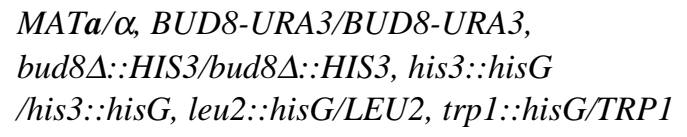 & this study \\
\hline RH2783 & 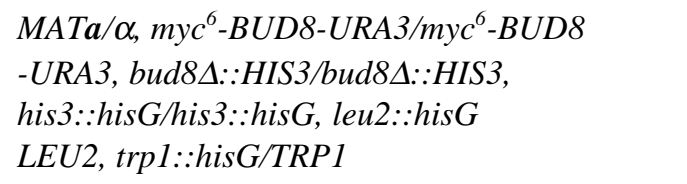 & this study \\
\hline RH2784 & 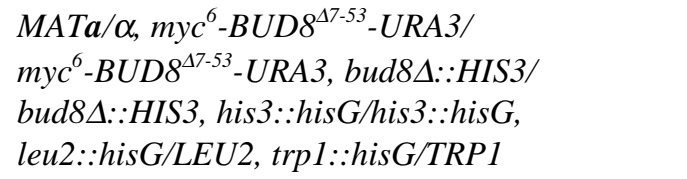 & this study \\
\hline RH2785 & 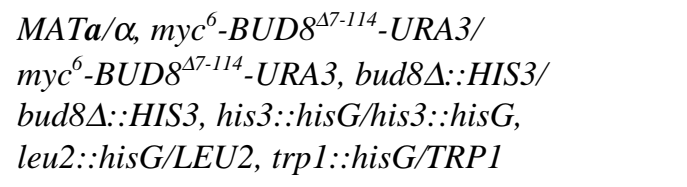 & this study \\
\hline RH2786 & $\begin{array}{l}\text { MATa/ } \alpha, \text { myc }^{6}-B U D 8^{47-417}-U R A 3 / \\
\text { /myc }{ }^{6}-B U D 8^{47-417}-U R A 3, \text { bud } 8 \Delta:: H I S 3 / \\
\text { bud } 8 \Delta:: H I S 3, \text { his } 3:: \text { hisG/his3::hisG, } \\
\text { leu2::hisG/LEU2, trp1::hisG/TRP1 }\end{array}$ & this study \\
\hline RH2787 & 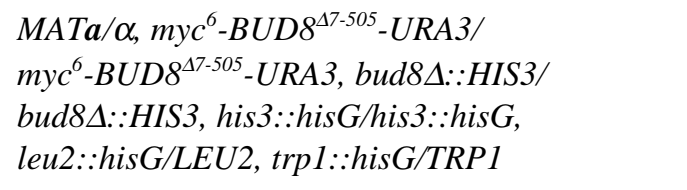 & this study \\
\hline RH2788 & 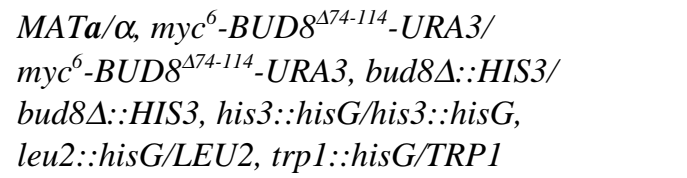 & this study \\
\hline RH2789 & 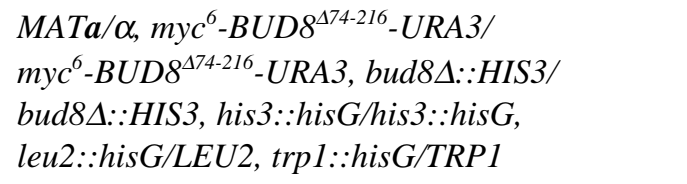 & this study \\
\hline RH2790 & 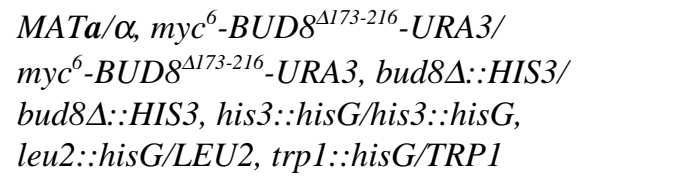 & this study \\
\hline
\end{tabular}


Table VII. Strains used in this study

\begin{tabular}{|c|c|c|}
\hline Strain & Genotype & Source \\
\hline RH2791 & 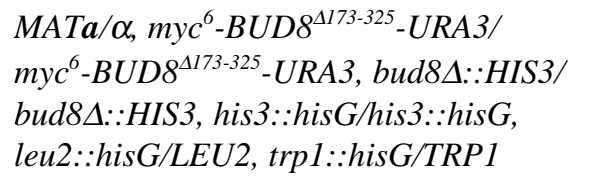 & this study \\
\hline RH2792 & 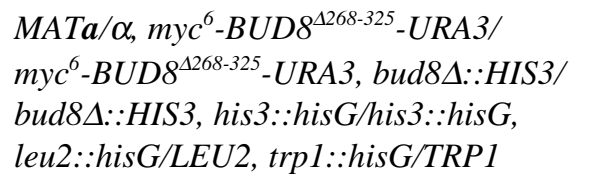 & this study \\
\hline RH2793 & 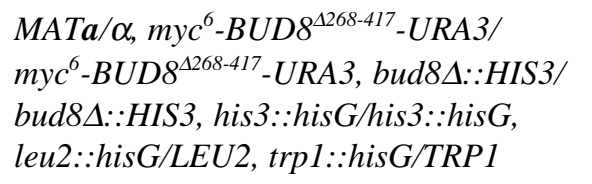 & this study \\
\hline RH2794 & 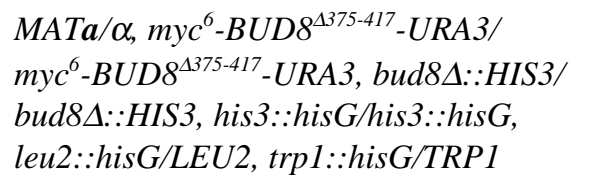 & this study \\
\hline RH2795 & 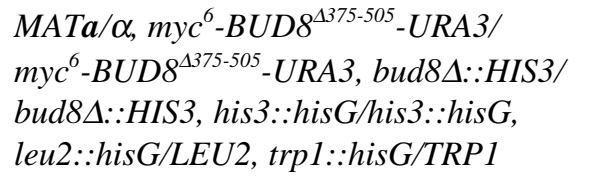 & this study \\
\hline RH2796 & 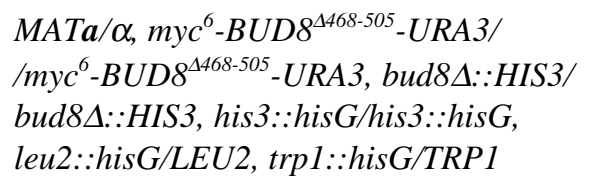 & this study \\
\hline RH2797 & 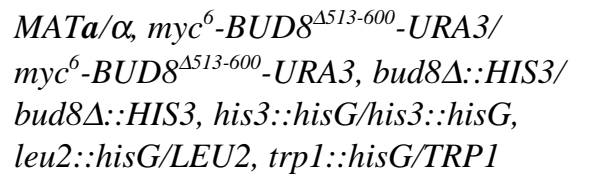 & this study \\
\hline RH2798 & 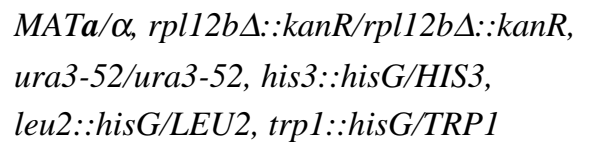 & this study \\
\hline
\end{tabular}


Table VIII. Plasmids used in this study

\begin{tabular}{|c|c|c|}
\hline Plasmid & Description & Reference \\
\hline pEG202 & $\begin{array}{l}\text { HIS3-marked } 2 \mu \mathrm{m} A D H 1 \text { prom-lexAD } \\
B D \text {-ADH1term two-hybrid vector }\end{array}$ & (Fashena et al., 2000) \\
\hline pJG4-5 & $\begin{array}{l}\text { TRP1-marked } 2 \mu \mathrm{m} \text { GAL1prom- } \\
\text { SV4ONLS- B42AD-ADH1term, two-hybrid vector }\end{array}$ & (Fashena et al., 2000) \\
\hline pJG4-5 library & S. cerevisiae cDNA library in pJG4-5 & (Fashena et al., 2000) \\
\hline pRS306 & URA3-marked integrative vector & (Sikorski and Hieter, 1989) \\
\hline pRS425 & $L E U 2$-marked $2 \mu \mathrm{m}$ vector & (Christianson et al., 1992) \\
\hline pRS426 & $U R A 3$-marked $2 \mu \mathrm{m}$ vector & (Christianson et al., 1992) \\
\hline pYGEX-2T & $U R A 3$-marked $2 \mu \mathrm{m}$, GAL1prom-GST fusion vector & (Schlenstedt et al., 1995) \\
\hline pYGEX-2T & $U R A 3$-marked $2 \mu \mathrm{m}$, GAL1prom-GST fusion vector & (Schlenstedt et al., 1995) \\
\hline pME1772 & BUD8prom-GFP-BUD8 fusion in pRS426 & (Taheri et al., 2000) \\
\hline pME1939 & BUD9prom-myc ${ }^{3}-B U D 9$ fusion in $\mathrm{pRS} 425$ & (Taheri et al., 2000) \\
\hline pME2247 & GAL1prom-GST-RPL12A fusion in pYGEX-2T & this study \\
\hline pME2248 & GAL1prom-GST-SCP160 fusion in pYGEX-2T & this study \\
\hline pME2336 & GAL1prom-GST-TRS120 fusion in pYGEX-2T & this study \\
\hline pME2252 & RPL12Aprom-myc ${ }^{3}-R P L 12 A$ fusion in pRS306 & this study \\
\hline pME2253 & SCP160prom-myc ${ }^{9}-S C P 160$ fusion in pRS306 & this study \\
\hline pME2254 & RPL12Aprom-GFP-RPL12A fusion in pRS306 & this study \\
\hline pME2255 & SCP160-prom-GFP-SCP160 fusion in pRS306 & this study \\
\hline pME2256 & pRS316 with novel $B g l \mathrm{II}$ restriction site in polylinker & this study \\
\hline pME2271 & $\begin{array}{l}354 \text { bp } R P L 12 A \text {-fragment in pJG4-5 } \\
\text { from cDNA library }\end{array}$ & this study \\
\hline pME2272 & $\begin{array}{l}756 \text { bp } S C P 160 \text {-fragment in pJG4-5 } \\
\text { from cDNA library }\end{array}$ & this study \\
\hline pME2273 & $\begin{array}{l}348 \text { bp TRS120-fragment in pJG4-5 } \\
\text { from cDNA library }\end{array}$ & this study \\
\hline pME2274 & $\begin{array}{l}\text { ADH1prom-lexADBD-BUD } 8^{2-512}- \\
\text { ADH1term in pEG202 }\end{array}$ & this study \\
\hline pME2318 & BUD8prom-myc $c^{6}-B U D 8$ in pRS426 & this study \\
\hline pME2297 & BUD8prom-myc $c^{6}-B U D 8^{\Delta 513-600}$ in pRS426 & this study \\
\hline pME2319 & BUD8prom-myc $c^{6}-B U D 8$ in pRS425 & this study \\
\hline pME2298 & BUD8prom-myc $c^{6}-B U D 8^{\Delta 513-600}$ in pRS425 & this study \\
\hline pME2302 & $\begin{array}{l}r p l 12 a \Delta:: k a n R \text { cassette for full } \\
\text { deletion of } R P L 12 A\end{array}$ & this study \\
\hline pME2303 & $\begin{array}{l}\operatorname{scp} 160 \Delta:: k a n R \text { cassette for full } \\
\text { full deletion of } S C P 160\end{array}$ & this study \\
\hline pME2320 & $B U D 8$ in $\mathrm{pRS} 306$ & this study \\
\hline pME2321 & BUD8prom-myc ${ }^{6}-B U D 8$ fusion in $\mathrm{pRS} 306$ & this study \\
\hline
\end{tabular}


Table VIII. Plasmids used in this study

\begin{tabular}{|c|c|c|}
\hline Plasmid & Description & Reference \\
\hline pME2322 & $B U D 8$ prom-myc ${ }^{6}-B U D 8^{\Delta 7-53}$ fusion in pRS306 & this study \\
\hline pME2323 & BUD8prom-myc ${ }^{6}-B U D 8^{\Delta 7-114}$ fusion in pRS306 & this study \\
\hline pME2324 & $B U D 8$ prom- $m y c^{6}-B U D 8^{\Delta 7-417}$ fusion in $\mathrm{pRS} 306$ & this study \\
\hline pME2325 & BUD8prom-myc ${ }^{6}-B U D 8^{\Delta 7-505}$ fusion in pRS306 & this study \\
\hline pME2326 & BUD8prom-myc $c^{6}-B U D 8^{\Delta 74-114}$ fusion in pRS306 & this study \\
\hline pME2327 & $B U D 8$ prom-myc $c^{6}-B U D 8^{\Delta 74-216}$ fusion in pRS306 & this study \\
\hline pME2328 & BUD8prom-myc ${ }^{6}-B U D 8^{\Delta 173-216}$ fusion in pRS306 & this study \\
\hline pME2329 & $B U D 8$ prom-myc $c^{6}-B U D 8^{\Delta 173-325}$ fusion in pRS306 & this study \\
\hline pME2330 & BUD8prom-myc $c^{6}-B U D 8^{\Delta 268-325}$ fusion in pRS306 & this study \\
\hline pME2331 & BUD8prom-myc $c^{6}-B U D 8^{\Delta 268-417}$ fusion in pRS306 & this study \\
\hline pME2332 & BUD8prom-myc $c^{6}-B U D 8^{\Delta 375-417}$ fusion in pRS306 & this study \\
\hline pME2333 & BUD8prom-myc $c^{6}-B U D 8^{\Delta 375-505}$ fusion in pRS306 & this study \\
\hline pME2334 & BUD8prom-myc $c^{6}-B U D 8^{\Delta 468-505}$ fusion in $\mathrm{pRS} 306$ & this study \\
\hline pME2335 & $B U D 8$ prom- $m y c^{6}-B U D 8^{\Delta 513-600}$ fusion in $\mathrm{pRS} 306$ & this study \\
\hline pME2424 & $r p l 12 b \Delta:: k a n R$ cassette for full deletion of $R P L 12 b$ & this study \\
\hline
\end{tabular}




\section{References}

Bandziulis, R.J., Swanson, M.S. and Dreyfuss, G. (1989) RNA-binding proteins as developmental regulators. Genes Dev., 3, 431-437.

Barrowman, J., Sacher, M. and Ferro-Novick, S. (2000) TRAPP stably associates with the Golgi and is required for vesicle docking. EMBO J., 19, 862-869.

Bauer, F., Urdaci, M., Aigle, M. and Crouzet, M. (1993) Alteration of a yeast SH3 protein leads to conditional viability with defects in cytoskeletal and budding patterns. Mol. Cell. Biol., 13, 5070-5084.

Bender, A. and Pringle, J.R. (1989) Multicopy suppression of the $c d c 24$ budding defect in yeast by $C D C 42$ and three newly identified genes including the rasrelated gene RSR1. Proc. Natl. Acad. Sci. USA, 86, 9976-9980.

Bobola, N., Jansen, R.-P., Shin, T.H. and Nasmyth, K. (1996) Asymetric accumulation of Ash1p in postanaphase nuclei depends on a myosin and restricts yeast mating-type switching to mother cells. Cell, 84, 699-709.

Briones, E., Briones, C., Remacha, M. and Ballesta, J.P. (1998) The GTPase center protein L12 is required for correct ribosomal stalk assembly but not for Saccharomyces cerevisiae viability. J. Biol. Chem., 273, 31956-31961.

Chant, J. (1999) Cell polarity in yeast. Annu. Rev. Cell Dev. Biol., 15, 365-391.

Chant, J., Corrado, K., Pringle, J.R. and Herskowitz, I. (1991) Yeast BUD5, encoding a putative GDP-GTP exchange factor, is necessary for bud site selection and interacts with bud formation gene BEM1. Cell, 65, 1213-1224.

Chant, J. and Herskowitz, I. (1991) Genetic control of bud site selection in yeast by a set of gene products that constitute a morphogenetic pathway. Cell, 65, 12031212.

Chant, J. and Pringle, J.R. (1991) Budding and cell polarity in Saccharomyces cerevisiae. Curr. Opin. Genet. Dev., 1, 342-350.

Chen, T., Hiroko, T., Chaudhuri, A., Inose, F., Lord, M., Tanaka, S., Chant, J. and Fujita, A. (2000) Multigenerational cortical inheritance of the Rax2 protein in orienting polarity and division in yeast. Science, 290, 1975-1978.

Christianson, T.W., Sikorski, R.S., Dante, M., Shero, J.H. and Hieter, P. (1992) Multifunctional yeast high-copy-number shuttle vectors. Gene, 110, 119-122.

Drees, B.L., Sundin, B., Brazeau, E., Caviston, J.P., Chen, G.C., Guo, W., Kozminski, K.G., Lau, M.W., Moskow, J.J., Tong, A., Schenkman, L.R., McKenzie, A., 3rd, Brennwald, P., Longtine, M., Bi, E., Chan, C., Novick, P., Boone, C., Pringle, J.R., Davis, T.N., Fields, S. and Drubin, D.G. (2001) A protein interaction map for cell polarity development. J. Cell Biol., 154, 549-571.

Fashena, S.J., Serebriiskii, I.G. and Golemis, E.A. (2000) LexA-based two-hybrid systems. Methods Enzymol., 328, 14-26. 
Frey, S., Pool, M. and Seedorf, M. (2001) Scp160p, an RNA-binding, polysomeassociated protein, localizes to the endoplasmic reticulum of Saccharomyces cerevisiae in a microtubule- dependent manner. J. Biol. Chem., 276, 15905-15912.

Fujita, A., Oka, C., Arikawa, Y., Katagai, T., Tonouchi, A., Kuhara, S. and Misumi, Y. (1994) A yeast gene necessary for bud-site selection encodes a protein similar to insulin-degrading enzymes. Nature, 372, 567-570.

Gimeno, C.J., Ljungdahl, P.O., Styles, C.A. and Fink, G.R. (1992) Unipolar cell divisions in the yeast $S$. cerevisiae lead to filamentous growth: regulation by starvation and RAS. Cell, 68, 1077-1090.

Guthrie, C. and Fink, G.R. (1991) Guide to Yeast Genetics and Molecular Biology. Methods Enzymol., 194.

Gyuris, J., Golemis, E., Chertkov, H. and Brent, R. (1993) Cdi1, a Human G1 and S Phase Protein Phosphatase That Associates with Cdk2. Cell, 75, 791-803.

Halme, A., Michelitch, M., Mitchell, E.L. and Chant, J. (1996) Bud10p directs axial cell polarization in budding yeast and resembles a transmembrane receptor. Curr. Biol., 6, 570-579.

Harkins, H.A., Page, N., Schenkman, L.R., De Virgilio, C., Shaw, S., Bussey, H. and Pringle, J.R. (2001) Bud8p and Bud9p, proteins that may mark the sites for bipolar budding in yeast. Mol. Biol. Cell, 12, 2497-2518.

Hoffman, C.S. and Winston, F. (1987) A ten-minute DNA preparation from yeast efficiently releases autonomous plasmids for transformation of Escherichia coli. Gene, 57, 267-272.

Irie, K., Tadauchi, T., Takizawa, P.A., Vale, R.D., Matsumoto, K. and Herskowitz, I. (2002) The Khd1 protein, which has three KH RNA-binding motifs, is required for proper localization of ASHI mRNA in yeast. EMBO J., 21, 1158-1167.

Ito, H., Fukuda, Y., Murata, K. and Kimura, A. (1983) Transformation of intact yeast cells treated with alkali cations. J. Bacteriol., 153, 163-168.

Ito, T., Chiba, T., Ozawa, R., Yoshida, M., Hattori, M. and Sakaki, Y. (2001) A comprehensive two-hybrid analysis to explore the yeast protein interactome. Proc. Natl. Acad. Sci. U.S.A., 98, 4569-4574.

Jones, H.D., Schliwa, M. and Drubin, D.G. (1993) Video microscopy of organelle inheritance and motility in budding yeast. Cell. Motil. Cytoskeleton, 25, 129-142.

Kang, P.J., Sanson, A., Lee, B. and Park, H.O. (2001) A GDP/GTP exchange factor involved in linking a spatial landmark to cell polarity. Science, 292, 1376-1378.

Kron, S.J., Styles, C.A. and Fink, G.R. (1994) Symmetric cell division in pseudohyphae of the yeast Saccharomyces cerevisiae. Mol. Biol. Cell, 5, 10031022.

Lang, B.D. and Fridovich-Keil, J.L. (2000) Scp160p, a multiple KH-domain protein, is a component of mRNP complexes in yeast. Nucleic Acids Res., 28, 1576-1584. 
Lang, B.D., Li, A., Black-Brewster, H.D. and Fridovich-Keil, J.L. (2001) The brefeldin A resistance protein Bfr1p is a component of polyribosome- associated mRNP complexes in yeast. Nucleic Acids Res., 29, 2567-2574.

Lord, M., Yang, M.C., Mischke, M. and Chant, J. (2000) Cell cycle programs of gene expression control morphogenetic protein localization. J. Cell Biol., 151, 1501-1512.

Mösch, H.-U. and Fink, G.R. (1997) Dissection of filamentous growth by transposon mutagenesis in Saccharomyces cerevisiae. Genetics, 145, 671-684.

Ni, L. and Snyder, M. (2001) A Genomic Study of the Bipolar Bud Site Selection Pattern in Saccharomyces cerevisiae. Mol. Biol. Cell, 12, 2147-2170.

Park, H.O., Bi, E., Pringle, J.R. and Herskowitz, I. (1997) Two active states of the Ras-related Bud1/Rsr1 protein bind to different effectors to determine yeast cell polarity. Proc. Natl. Acad. Sci. USA, 94, 4463-4468.

Park, H.O., Chant, J. and Herskowitz, I. (1993) BUD2 encodes a GTPase-activating protein for Bud1/Rsr1 necessary for proper bud-site selection in yeast. Nature, 365, 269-274.

Park, H.O., Sanson, A. and Herskowitz, I. (1999) Localization of Bud2p, a GTPaseactivating protein necessary for programming cell polarity in yeast to the presumptive bud site. Genes Dev., 13, 1912-1917.

Pringle, J.R. (1991) Staining of bud scars and other cell wall chitin with calcofluor. Methods Enzymol., 194, 732-735.

Pringle, J.R., Adams, A.E., Drubin, D.G. and Haarer, B.K. (1991) Immunofluorescence methods for yeast. Methods Enzymol., 194, 565-602.

Pucciarelli, M.G., Remacha, M., Vilella, M.D. and Ballesta, J.P. (1990) The 26S rRNA binding ribosomal protein equivalent to bacterial protein L11 is encoded by unspliced duplicated genes in Saccharomyces cerevisiae. Nucleic Acids Res., 18, 4409-4416.

Rechsteiner, M. and Rogers, S.W. (1996) PEST sequences and regulation by proteolysis. Trends Biochem. Sci., 21, 267-271.

Roberts, R.L., Mösch, H.-U. and Fink, G.R. (1997) 14-3-3 proteins are essential for RAS/MAPK cascade signaling during pseudohyphal development in $S$. cerevisiae. Cell, 89, 1055-1065.

Roemer, T., Madden, K., Chang, J. and Snyder, M. (1996) Selection of axial growth sites in yeast requires Axl2p, a novel plasma membrane glycoprotein. Genes Dev., 10, 777-793.

Rose, M. and Botstein, D. (1983) Construction and use of gene fusions to lacZ ( $\beta$ galactosidase) that are expressed in yeast. Methods Enzymol., 101, 167-180.

Sacher, M., Barrowman, J., Schieltz, D., Yates, J.R., 3rd and Ferro-Novick, S. (2000) Identification and characterization of five new subunits of TRAPP. Eur. J. Cell Biol., 79, 71-80. 
Sanders, S.L. and Herskowitz, I. (1996) The BUD4 protein of yeast, required for axial budding, is localized to the mother/BUD neck in a cell cycle-dependent manner. J. Cell Biol., 134, 413-427.

Schenkman, L.R., Caruso, C., Page, N. and Pringle, J.R. (2002) The role of cell cycle-regulated expression in the localization of spatial landmark proteins in yeast. J. Cell Biol., 156, 829-841.

Schlenstedt, G., Saavedra, C., Loeb, J.D., Cole, C.N. and Silver, P.A. (1995) The GTP-bound form of the yeast Ran/TC4 homologue blocks nuclear protein import and appearance of poly(A)+ RNA in the cytoplasm. Proc. Natl. Acad. Sci. U.S.A., 92, 225-229.

Sheu, Y.J., Barral, Y. and Snyder, M. (2000) Polarized growth controls cell shape and bipolar bud site selection in Saccharomyces cerevisiae. Mol. Cell. Biol., 20, 5235-5247.

Sikorski, R.S. and Hieter, P. (1989) A system of shuttle vectors and yeast host strains designed for efficient manipulation of DNA in Saccharomyces cerevisiae. Genetics, 122, 19-27.

Snyder, M. (1989) The Spa2p protein of yeast localizes to sites of cell growth. J. Cell Biol., 108, 1419-1429.

Taheri, N., Köhler, T., Braus, G.H. and Mösch, H.-U. (2000) Asymmetrically localized Bud8p and Bud9p proteins control yeast cell polarity and development. EMBO J., 19, 6686-6696.

Wang, W., Sacher, M. and Ferro-Novick, S. (2000) TRAPP stimulates guanine nucleotide exchange on Ypt1p. J. Cell Biol., 151, 289-296.

Winzeler, E.A., Shoemaker, D.D., Astromoff, A., Liang, H., Anderson, K., Andre, B., Bangham, R., Benito, R., Boeke, J.D., Bussey, H., Chu, A.M., Connelly, C., Davis, K., Dietrich, F., Dow, S.W., El Bakkoury, M., Foury, F., Friend, S.H., Gentalen, E., Giaever, G., Hegemann, J.H., Jones, T., Laub, M., Liao, H., Davis, R.W. and et al. (1999) Functional characterization of the $S$. cerevisiae genome by gene deletion and parallel analysis. Science, 285, 901-906.

Zahner, J.E., Harkins, H.A. and Pringle, J.R. (1996) Genetic analysis of the bipolar pattern of bud site selection in the yeast Saccharomyces cerevisiae. Mol. Cell. Biol., 16, 1857-1870. 


\section{Curriculum Vitae}

\section{Naimeh Taheri Talesh}

Date and Place of Birth:

September 11th, 1969 in Talesh / Iran

Educational Background:

$\begin{array}{ll}1975-1980 & \text { Primary School in Assalem / Iran } \\ 1980-1983 & \text { Middle School in Assalem / Iran } \\ 1983-1987 & \begin{array}{l}\text { Secondary School in Talesh / Iran } \\ \text { Certificate of Secondary education }\end{array}\end{array}$

$1988-1989$ Courses in German language at the Volkshochschule

(VHS)

$1990-1991 \quad$ University preparatory year for foreign students at the University of Hannover / Germany

$1991-1996 \quad$ Study of Biology at the Georg-August-University of Göttingen / Germany

1996 - 1997 Dissertation for academic degree: Diplom Topic: Isolation and characterisation of Poly 3Hydroxyoctanoic-Depolymerase from Pseudomonas fluorescens GK13

Supervisor : PD Dr. Dieter Jendrossek at the Institute of Microbiology at the University of Göttingen

October 1997

Academic degree: Diplom in Biologie

Since 1998

Research assictant/doctoral candidate at the Institute of Microbiology and Genetics at the University of Göttingen Topic: Regulation of cell polarity in the budding yeast Saccharomyces cerevisiae Supervisor: PD Dr. Hans-Ulrich Mösch 